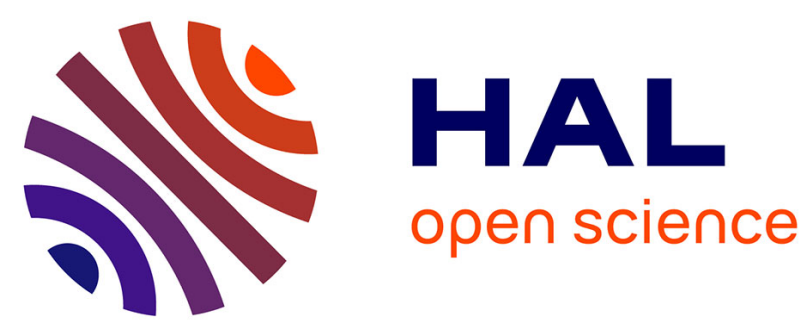

\title{
Pressure-Leak Duality for Leak Detection and Localization in Water Distribution Systems
}

David Steffelbauer, Jochen Deuerlein, Denis Gilbert, Edo Abraham, Olivier Piller

\section{- To cite this version:}

David Steffelbauer, Jochen Deuerlein, Denis Gilbert, Edo Abraham, Olivier Piller. Pressure-Leak Duality for Leak Detection and Localization in Water Distribution Systems. Journal of Water Resources Planning and Management, 2022, 148 (3), 10.1061/(ASCE)WR.1943-5452.0001515 . hal-03517648

\section{HAL Id: hal-03517648 \\ https://hal.inrae.fr/hal-03517648}

Submitted on 7 Jan 2022

HAL is a multi-disciplinary open access archive for the deposit and dissemination of scientific research documents, whether they are published or not. The documents may come from teaching and research institutions in France or abroad, or from public or private research centers.
L'archive ouverte pluridisciplinaire HAL, est destinée au dépôt et à la diffusion de documents scientifiques de niveau recherche, publiés ou non, émanant des établissements d'enseignement et de recherche français ou étrangers, des laboratoires publics ou privés. 
Author-produced version of the article published in Journal of Water Resources Planning and Management, 148(3), 04021106. The original publication is available at

https://ascelibrary.org/doi/full/10.1061/(ASCE)WR.1943-5452.0001515 


\title{
PRESSURE-LEAK DUALITY FOR LEAK DETECTION AND LOCALIZATION IN WATER DISTRIBUTION SYSTEMS
}

\author{
David B. Steffelbauer ${ }^{1,2}$, Jochen Deuerlein ${ }^{3,5}$, Denis Gilbert ${ }^{4}$, Edo Abraham ${ }^{2}$, and Olivier Piller ${ }^{4,5}$ \\ ${ }^{1}$ Department of Civil and Environmental Engineering, Norwegian Univ. of Science and \\ Technology (NTNU), S.P. Andersens veg 5, 7031 Trondheim, Norway. Email: \\ david.steffelbauer@ntnu.no \\ ${ }^{2}$ Water Management Department, TU Delft, Stevinweg 1, 2628 CN, The Netherlands. \\ 33S Consult GmbH, Albtalstrasse 13, 76137 Karlsruhe, Germany \\ ${ }^{4}$ INRAE, ETBX Research Unit, Aqua Department, F-33612 Cestas, France \\ ${ }^{5}$ School of Civil, Environmental and Mining Engineering, University of Adelaide, South Australia \\ 5005, Australia
}

\begin{abstract}
Water utilities are challenged to reduce their water losses through detecting, localizing, and repairing leaks as fast as possible in their aging distribution systems. In this work, we solve this challenging problem by detecting multiple leaks simultaneously in a water distribution network for the Battle of the Leak Detection and Isolation Methods. The performance of leak detection and localization depends on how well the system roughness and demand are calibrated. In addition, existing leaks affect the diagnosis performance unless they are identified and explicitly represented in the model. To circumvent this "chicken-and-egg" dilemma, we decompose the problem into multiple levels of decision making (a hierarchical approach) where we iteratively improve the water distribution network model and so are able to solve the multi-leak diagnosis problem.

First, a combination of time series and cluster analysis is used on smart meter data to build patterns for demand models. Second, point and interval estimates of pipe roughnesses are retrieved
\end{abstract}


using least squares to calibrate the hydraulic model, utilizing the demand models from the first step. Finally, the calibrated primal model is transformed into a dual model that intrinsically combines sensor data and network hydraulics. This dual model automatically converts small pressure deviations caused by leaks into sharp and localized signals in the form of virtual leak flows. Analytical derivations of sensitivities with respect to these virtual leak flows are calculated and used to estimate the leakage impulse responses at candidate nodes. Subsequently, we use the dual network to (i) detect the start time of the leaks and (ii) compute the Pearson correlation of pressure residuals, which allows further localization of leaks. This novel dual modeling approach resulted in the highest true-positive rates for leak isolation among all participating teams in the competition.

\section{INTRODUCTION}

The detection, localization and control of leakage from aging water distribution networks (WDNs) remains one of the main challenges for water utilities (WUs), because the direct financial cost of water loss can be high. By detecting and dealing with leaks and bursts fast, utilities can also mitigate deterioration of pipes and surrounding infrastructure in addition to lost revenues (Gupta and Kulat 2018). The aim to reduce leakage is further motivated by stringent regulations and financial incentives (OECD 2016).

Conventional techniques for detecting leakage include random and regular sounding surveys using listening sticks and acoustic loggers (Adedeji et al. 2017), and step-testing of metered subsystems as district metered areas (DMAs) through gradual valve closures (Farley and Trow 2003; Wu 2008). More advanced leakage pin-pointing methods like leak noise correlators, pigmounted acoustic sensing and gas-injection techniques (Puust et al. 2010) are the most precise at locating leaks. However, all these techniques come with expensive equipment cost and are manhour intensive, and so are not scalable. In addition, the suppression of leakage sound signatures by reduced pressures in active pressure management or increasing use of plastic pipes in the network has also made these methods less effective (Wu 2008; Puust et al. 2010).

More recent advanced approaches use model-based analysis of near real-time telemetry data 
from pressure sensors and flow meters distributed over the network. Starting with the work of Pudar and Liggett 1992, model-based leak localization was intensively studied with diverse set of methods ranging from sensitivity matrix-based approaches (Pérez et al. 2011; Perez et al. 2014), meta-heuristic optimization (Wu 2008; Steffelbauer and Fuchs-Hanusch 2016b), error-domain model falsification (Goulet et al. 2013; Moser et al. 2017), to combinations of model-based and data-driven approaches (Soldevila et al. 2016; Soldevila et al. 2017). An extensive review of leak localization techniques including their limitations can be found in $\mathrm{Hu}$ et al. 2021. This manuscript deals with a novel model-based approach that leverages time-series analysis of demand models and new hydraulic modeling approaches for both detecting and localizing potential leaks. One of the main challenges for model-based leak detection approaches is the sparse number of pressure sensors compared to the number of candidate leak location nodes. For methods that solve for multiple leaks by posing inverse problems to determine leak parameters in the network model (Pudar and Liggett 1992) (i.e. leak levels and locations), this creates an under-determined and ill-posed problem. Additionally, the performance of model-based approaches can also be very sensitive to errors in two important model parameters: the demand at nodes and pipe roughness coefficients (Hutton et al. 2014). Sanz et al. 2016 reduce this error by including existing leaks in the calibration process. This is done by co-optimizing the calibration and detection, and updating the calibrated model through iteration as new data becomes available and leaks are discovered and fixed. This is achieved through an iterative calibration process, where demands at nodes are composed of geographically distributed demand components. Due to the fact that a leak occurs as a less geographically spread component in this approach, they become easier to find. The method of Sanz et al. 2016 belongs to a class of methods that rely on first-order pressure sensitivities to changes in demand at nodes, and the projection of pressure residuals (differences of measured pressures from leak free case, usually retrieved from time series or well calibrated hydraulic models) onto the sensitivities (Sanz et al. 2016). However, this class of methods have the limitations that they assume a single leak in the system at one time, and are known to be less reliable for small leak sizes, since the leak induced pressure deviations and, hence, the pressure residuals are very small in that case. 
In this manuscript, we address these limitations of pressure residual projection approaches (i.e., the applicability on single as well as small leaks) by combining multiple methods. As in Sanz et al. 2016, we utilize an iterative calibration of the system roughness and demand parameters using multiple measurements, including automatic meter readings (AMRs). To deal with multiple leaks, we separate the detection and localization process; time series analysis (TSA) is used to automatically find deviations in demand and flow measurements, thus, estimating the start and end time of multiple growing and non-growing leaks that can coincide. The detected leaks are then localized by using a residual projection approach (Steffelbauer et al. 2020), where the model is updated when leaks are discovered or fixed. A new duality-based approach is then proposed to improve the sensitivity of the localization process to smaller leaks. We formulate a dual network model, where thanks to a mathematical trick — by transforming the network model with pressure measurements to an equivalent model with additional virtual reservoirs and valves — we are able to translate pressure heads directly to virtual leakage outflows at the measurement locations, which provide a first estimate for the leak's size and location in the network.

Subsequently, we use the virtual leak flows of the dual model for leak detection with anomaly detection algorithms (i.e., the cumulative sum control chart (CUSUM) algorithm and the likelihood ratio test (Peach et al. 1995)) to obtain information on the leak start-time; and the residual-based localization to retrieve the location of the leak. Finally, the information from the detection and localization methods are combined to get accurate estimates for the actual size and location of the leaks.

In the next section, an exposition of the different methods will be presented. We will then discuss the results using the L-Town network model of the Battle of the Leak Detection and Isolation Methods (BattLeDIM) competition (Vrachimis et al. 2020), which the authors of this manuscript won under the team name Under Pressure. The final section will present the conclusions, limitations and future directions to improve the proposed method.

\section{METHODS}




\section{Overview}

We solve the leak detection and isolation problem through utilizing a hierarchical approach. An overview of the two stages where different methods are combined as well as the order in which they are applied is illustrated in Figure 1, depicting how we attempted to find leaks in the measurement data via model calibration and then simulation with the dual model. In the first stage, the hydraulic model is calibrated, since a well-calibrated model is essential to reliably localize leaks (Savic et al. 2009). The model is itself calibrated in two-stages; starting with demand calibration and followed by pipe roughness parameter estimation. The demand calibration method makes use of TSA on AMR data $d$, and infers estimated demands $\hat{d}$ to unmeasured nodes with respect to their average demand $\bar{d}$ stored in the EPANET file. The pipe roughnesses $\hat{C}$ are estimated through solving a differentiable, constrained, weighted least squares (WLS) problem, which uses the estimated demands $\hat{d}$, measured pressure heads $h$, and the initial roughness values $C$ as found in the original EPANET file. In the second, a dual model is built based on the calibrated values $(\hat{d}$ and $\hat{C})$ and used for leak detection and localization, where pressure measurements are replaced with virtual reservoirs. The dual model magnifies leak signals by transforming pressures in virtual leakage outflows $q_{v}$. Moreover, dual model leak sensitivities $S$ are computed. Finally, the sensitivities $S$ and virtual flows $q_{v}$ are used to locate the leaks with a correlation-based method similar to Sanz et al. 2016. In cases with multiple leaks that appear simultaneously, the leaks are localized one by one, eliminated from the dual model, and the remaining leaks are detected and located subsequently through an iterative approach.

\section{Calibration}

\section{Nodal demand calibration}

The AMRs data is used to develop a demand model through TSA for the unmeasured customers within the network. Various time series models (Shumway and Stoffer 2010) are tested on the AMRs aiming to extract weekly seasonalities and yearly trends for different customer types (e.g., residential, commercial). The best performance is achieved with a rather simple model, consisting of a multiplicative superposition of weekly seasonalities $(S(t))$, a time varying trend $(T(t))$ and a 
random component $(R(t))$ accounting for stochastic variations and measurement noise

$$
d(t)=\bar{d} \cdot T(t) \cdot S(t) \cdot R(t)
$$

with $\bar{d}$ being the customer's base demand. For each AMR time series, the trend component $T(t)$ is estimated using a convolution filter and subsequently removed by dividing the original time series through $T(t)$, followed by estimation of $S(t)$ through periodical averages over the trend-free series (Seabold and Perktold 2010). After removing the seasonal component by dividing the trendfree series by $S(t)$, only the random component $R(t)$ remains. Subsequently, similarities in the individual seasonal patterns are identified through time series clustering (Steffelbauer et al. 2021). Furthermore, cluster analysis is used to identify the number of distinct patterns $n_{d}$ and outliers. For each demand node $i$ of the network model, a time-varying demand time series $\hat{d}_{i}$ is built as a superposition of the distinct patterns weighted by their individual averages $\bar{d}_{i j}$ associated with the patterns

$$
\hat{d}_{i}(t)=\sum_{j=1}^{n_{d}} \bar{d}_{i j} \cdot T_{j}(t) \cdot S_{j}(t)
$$

Note that the random time series components are neglected when building the estimates $\hat{d}_{i}$.

\section{Pipe roughness calibration}

Pipes with the same material, age, diameter, hydraulic conditions and locations are grouped in clusters with the same roughness value (in this case a Hazen-Williams (HW) coefficient)

$$
\mathbf{C}_{H W}=\mathbf{M}_{H W} \mathbf{x}
$$

where $\mathbf{M}_{H W}$ is the membership matrix of the $n_{p}$ pipes to $n_{c}$ clusters of $\mathrm{HW}$ coefficients, $\mathbf{x} \in \mathbb{R}^{n_{c}}$ is the vector of roughness cluster values to calibrate, and $\mathbf{C}_{H W} \in \mathbb{R}^{n_{p}}$ is the vector of $\mathrm{HW}$ coefficients of pipes. Roughness calibration aims to fit the measurements by adjusting the roughness coefficients 
of the hydraulic model. Following nonlinear regression equations have to be considered

$$
\left[\mathbf{z}^{j}\right]_{i}=\left[\mathbf{S} \mathbf{y}\left(t_{j}, \mathbf{x}\right)\right]_{i}+\varepsilon_{i j}
$$

where $\mathbf{y}\left(t_{j}, \mathbf{x}\right)$ is the hydraulic state that is implicitly defined by the extended period simulations at time $t_{j}, \mathbf{z}^{\mathbf{j}} \in \mathbb{R}^{n_{m}}$ is the vector of measurements at time $t_{j}, \mathbf{S}$ is the selection matrix to select state vectors that correspond to the measurements, and $\varepsilon_{i j} \sim \mathcal{N}\left(0, \sigma_{i j}{ }^{2}\right)$ are independent and identically distributed Gaussian error terms with zero expectation and standard deviation $\sigma_{i j}$.

The box-constrained WLS problem for parameter calibration consists of seeking to minimize the differentiable criterion

$$
\min _{\mathbf{x}^{L} \leqslant \mathbf{x} \leqslant \mathbf{x}^{U}} \mathrm{f}(\mathbf{x}) \triangleq \frac{1}{2} \sum_{j=1}^{n_{t}} \sum_{i=1}^{n_{m}} \mathrm{H}_{\kappa}\left(\frac{\left[\mathbf{S y}\left(t_{j}, \mathbf{x}\right)\right]_{i}-\left[\mathbf{z}^{j}\right]_{i}}{\sigma_{i j}}\right)+\frac{\alpha}{2}\left\|\mathbf{x}-\mathbf{x}^{0}\right\|_{2}{ }^{2},
$$

where in place of the traditional least-squares criterion the weighted Huber function $\mathrm{H}_{\kappa}$ with parameter $\kappa$ is used, as in Preis et al. (2011), to increase the robustness of parameter estimates against outliers, $n_{t}$ is the number of observation times, $n_{m}$ the number of measurements, $\mathbf{x}^{L}$ and $\mathbf{x}^{U}$ are the lower and upper bounds, $\mathbf{x}^{0}$ is prior information about $\mathbf{x}(e . g$. initial value in the EPANET file) and $\alpha$ is a Tikhonov regularization coefficient, which penalizes large departures from $\mathbf{x}^{0}$ for sufficiently large $\alpha$ and increases the robustness of parameter estimates against outliers. The state of the art algorithm for solving a differentiable WLS problem is the iterative Levenberg-Marquardt algorithm. At each iteration step, the gradient of $\mathrm{f}$ is calculated to estimate the Hessian at the last estimate $\mathbf{x}^{k}$. The gradient of $\mathrm{f}$ at $\mathbf{x}^{k}$ is:

$$
\nabla \mathrm{f}^{k}=\sum_{j=1}^{n_{t}} \mathbf{J}\left(t_{j}, \mathbf{x}^{k}\right)^{T} \mathbf{W}_{j} \tilde{\mathbf{R}}\left(t_{j}, \mathbf{x}^{k}\right)+\alpha\left(\mathbf{x}^{k}-\mathbf{x}^{0}\right)
$$

where $\mathbf{W}_{j}$ is the diagonal weight matrix at time $t_{j}, \mathbf{J}\left(t_{j}, \mathbf{x}^{k}\right)=\mathbf{S} \partial_{x} \mathbf{y}\left(t_{j}, \mathbf{x}^{k}\right)$ is the Jacobian matrix of the prediction function at $\mathbf{x}^{k}$, with $\partial_{x} \mathbf{y}$ using the postmultiplication by $\mathbf{P}=\mathbf{M}_{H W}$ as in Piller et al. 
${ }_{167}$ (2017), and $\tilde{\mathbf{R}}\left(t_{j}, \mathbf{x}^{k}\right)$ is the $\left(n_{m}, 1\right)$-vector of truncated unreduced residuals

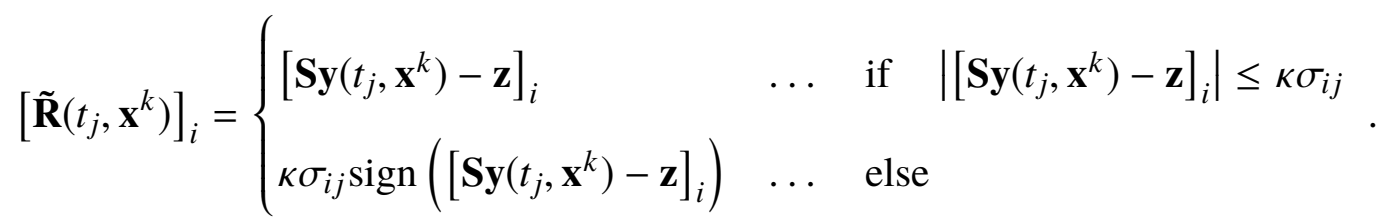

The estimate of the Hessian is following symmetric, positive definite matrix:

$$
\mathbf{H}_{k}=\sum_{j=1}^{n_{t}} \mathbf{J}\left(t_{j}, \mathbf{x}^{k}\right)^{T} \mathbf{W}_{j} \tilde{\mathbf{J}}\left(t_{j}, \mathbf{x}^{k}\right)+\alpha \mathbf{I}_{n c}=\sum_{j=1}^{n_{t}} \tilde{\mathbf{J}}\left(t_{j}, \mathbf{x}^{k}\right)^{T} \mathbf{W}_{j} \tilde{\mathbf{J}}\left(t_{j}, \mathbf{x}^{k}\right)+\alpha \mathbf{I}_{n c},
$$

where $\tilde{\mathbf{J}}$ is given by

$$
\left[\tilde{\mathbf{J}}\left(t_{j}, \mathbf{x}^{k}\right)\right]_{m n}=\left\{\begin{array}{lll}
{\left[\mathbf{J}\left(t_{j}, \mathbf{x}^{k}\right)\right]_{m n}} & \ldots & \text { if }\left|\left[\mathbf{S y}\left(t_{j}, \mathbf{x}^{k}\right)-\mathbf{z}\right]_{m}\right| \leq \kappa \sigma_{m j} \\
0 & \ldots & \text { else }
\end{array}\right.
$$

The constraints are taken into account through a saturation/desaturation process by checking the Karush-Kuhn-Tucker optimality conditions to identify the optimal Lagrange multipliers.

The projected Levenberg-Marquardt algorithm consists of solving following linear system

$$
\mathbf{x}^{k+1}=\mathbf{x}^{k}-\mathbf{C}_{k}^{T}\left(\mathbf{C}_{k} \mathbf{H}_{k} \mathbf{C}_{k}^{T}\right)^{-1} \mathbf{C}_{k} \nabla \mathbf{f}_{k}
$$

where $\mathbf{C}_{k}$ is the selection matrix for the unsaturated components $\mathbf{x}^{k}$. To cope with ill conditioned Hessians, a damping factor with a regularization parameter is introduced to scale the gradient according to the curvature

$$
\mathbf{H}_{k}(\lambda)=\mathbf{H}_{k}+\lambda\left[\operatorname{diag}\left(\mathbf{H}_{k}\right)+\phi \mathbf{I}_{n_{c}}\right]
$$

where $\phi$ is a positive parameter and $\lambda$ is the damping parameter. Furthermore, we make use of 
following relation to calculate confidence intervals for the roughness estimates (Piller 2019)

$$
-\left[\boldsymbol{\Delta}_{x}\right]_{i} \leqslant \delta \mathbf{x}_{i} \leqslant\left[\Delta_{x}\right]_{i}, \text { with } \mathbf{M}=\left(\mathbf{W}^{0.5} \mathbf{J}\right)^{+},\left[\boldsymbol{\Delta}_{x}\right]_{i}=3 \sum_{k=1}^{n_{m}}\left|\mathbf{M}_{i k}\right|, \mathrm{i}=1, \cdots, n_{c}
$$

with $\mathbf{J}$ is the block matrix $\mathbf{J}=\left(\begin{array}{lll}\mathbf{J}\left(t_{1}, \mathbf{x}\right)^{T} & \cdots & \mathbf{J}\left(t_{n_{t}}, \mathbf{x}\right)^{T}\end{array}\right)^{T}$ and $\mathbf{W}^{0.5}$ is the diagonal matrix $\mathbf{W}^{0.5}=\left(\mathbf{W}_{j}^{0.5}\right)=\left(\sigma_{i j}\right)$.

\section{The Dual Model}

We introduce a so-called "Dual Approach (DA)" for detecting and localizing leaks, that is depicted in Figure 2 (b). In the DA, the model is augmented with $n_{s}$ virtual reservoirs that are connected with pressure measurement nodes by valves. The origin of the name "dual" stems from the fact that, instead of using the fixed demand boundary condition at the sensor nodes (i.e. the original or "primal" hydraulic model), the measured pressure heads are used as fixed head boundary conditions at the corresponding virtual reservoirs. Consequently, the heads at the measurement nodes become free variables and imbalances in the system compared to a leak-free model lead to flows to the virtual reservoirs. If there are no leaks, and if we set the minor loss of each virtual reservoir's valve to zero, the two networks are equivalent. In the hydraulic model, we normally set these valves' minor loss to a sufficiently low but non-zero value, and so the primal and dual networks are 'numerically equivalent' but not mathematically equivalent.

If a new leak appears in the primal model, the residuals between measured and calculated pressures change. The pressure drops caused by higher flow velocities towards the leak in the real system are not observed in the model that is still based on the leak free system. In the dual approach, the measured pressure drop is applied to the fixed head reservoirs and, as a consequence, an additional outflow is generated. This outflow can be understood as an outflow residual or virtual leak flow. The advantage of the DA is that the calculated outflows act as amplifiers that deliver significant and localized signals even for small pressure drops. In addition, the outflows at the virtual reservoirs serve a good first estimate for the leak's size and location. 


\section{Dual Model Sensitivities}

We consider the dual WDN with $n_{p}$ pipes, $n_{s}$ virtual links and $n_{j}$ junction nodes at which the heads are unknown. We also denote the vector of unknown flows in the pipes and virtual links by $\mathbf{q} \in \mathbb{R}^{n_{p}+n_{s}}$, the unknown heads and demands at the (free) nodes by $\mathbf{h} \in \mathbb{R}^{n_{j}}$ and $\mathbf{d} \in \mathbb{R}^{n_{j}}$, respectively. The sensitivities of heads and pipe flow rates with respect to nodal outflows are derived among other sensitivities in Piller et al. (2017). The local sensitivities $\nabla_{\mathbf{d}} \mathbf{h}$ and $\nabla_{\mathbf{d}} \mathbf{q}$ can be calculated in demand driven analysis as follows

$$
\begin{aligned}
& \nabla_{\mathbf{d}} \mathbf{h}=-\left(\mathbf{A}^{T} \mathbf{F}^{-\mathbf{1}} \mathbf{A}\right)^{-1} \\
& \nabla_{\mathbf{d}} \mathbf{q}=-\mathbf{F}^{-1} \mathbf{A}\left(\mathbf{A}^{T} \mathbf{F}^{-\mathbf{1}} \mathbf{A}\right)^{-1},
\end{aligned}
$$

where $\mathbf{A}$ is the link-node-incidence matrix of the dual network graph reduced to junction nodes (all links, including pipes and virtual links, are taken), and $\mathbf{F}$ is the diagonal matrix of head loss derivatives with respect to $\mathbf{q}$.

Let $\mathbf{A}_{f} \in \mathbb{R}^{\left(n_{p}+n_{s}\right) \times\left(n_{f}+n_{s}\right)}$ be the link-node-incidence matrix of the dual network graph reduced to fixed-head nodes (the $n_{f}$ initial tanks and reservoirs, and the $n_{s}$ virtual reservoirs), and let $\mathbf{q}_{\text {in }}=\mathbf{A}_{f} \mathbf{q}$ represent the unknown flow rate entering in the system (leaving the fixed-head nodes if positive). Then the sensitivity of the $\mathbf{q}_{\text {in }}$ can be written as using Eq. (13)

$$
\nabla_{\mathbf{d}} \mathbf{q}_{i n}=-\mathbf{A}_{f}^{\mathbf{T}} \mathbf{F}^{-\mathbf{1}} \mathbf{A}\left(\mathbf{A}^{\mathbf{T}} \mathbf{F}^{-\mathbf{1}} \mathbf{A}\right)^{-\mathbf{1}}
$$

The Jacobian in Eq. (14) is the matrix of first order derivatives of the inflows calculated at virtual pressure nodes at measurement locations and real pressure boundary conditions such as reservoirs. The $(i, j)$ element of $\nabla_{\mathbf{d}} \mathbf{q}_{\text {in }}$ represents the first order change rate of the calculated in- or outflow at a fixed-head node $i$ as a consequence of a change in demand at node $j$.

In the dual model the in- and outflows at virtual reservoir are an indicator for a real existing leak or model errors. In a perfect model, where all the parameters are known, the calculated pressures of the dual model would be exactly the same as the measurements from a primal model. In the 
corresponding dual model, the calculated in- and outflows at junctions would be zero and the primal and the dual models would give approximately the same results (i.e. except for small numerical differences due to the minor losses across the virtual reservoir valves).

In presence of an unknown leak, the measured pressure heads and the values calculated by the leak-free primal model diverge. In the dual model, the pressures at the measurement nodes become free and the measurements are set as virtual fixed heads (Figure 2 (b)). The imbalance caused by the unknown leak is then expressed as in- and outflows calculated at pressure measurement nodes. However, as we have shown in the BattLeDIM (Steffelbauer et al. 2020), the sensitivity is much higher in the dual model. Inverting the problem acts as an amplifier of leaks. Another advantage is that the imbalances and the value in question (leaks) have the same unit of flow. The sum of all the imbalances normally gives a good first estimate of the size of the leak. For explanation of the amplifying effect, a deeper investigation of the equation (14) may be useful: from the balance of inflows and outflows, it is possible to deduce each column of $\nabla_{\mathbf{d}} \mathbf{q}_{\text {in }}$ including the fraction of inand outflows as a response to the change in outflow at the corresponding demand node equation

$$
\mathbf{1}_{n_{f}+n_{s}}{ }^{T} \mathbf{q}_{i n}=\mathbf{1}_{n_{j}}{ }^{T} \mathbf{d} \Rightarrow \mathbf{1}_{n_{f}+n_{s}}{ }^{T} \nabla_{\mathbf{d}} \mathbf{q}_{i n}=\mathbf{1}_{n_{j}}{ }^{T} .
$$

The sum of the column vector must be one. Naturally, the response should be an inflow for all fixed-head nodes.

\section{Leak detection and localization}

\section{Leak detection with the dual model}

Whereas in the past, human operators were in charge of small single supply areas, modern WU employees are responsible for multiple DMAs simultaneously (Bakker et al. 2014). That is why automatic anomaly detection algorithms are of particular interest for providing a rapid response to leaks and pipe burst (Romano et al. 2013). However, a correct estimation of the total leakage outflow over their time of existence (from the start $t_{S}$ until the end $t_{E}$ when they are repaired) is of utmost importance to assess water losses (Hamilton and McKenzie 2014). The correct identification of 
$t_{S}$ is also one of the objectives in the BattLeDIM (Vrachimis et al. 2020). We developed a twostage approach to tackle both tasks: (i) using anomaly detection algorithms to detect leaks as fast as possible, and (ii) using regression analysis to retrieve good leak start time $t_{S}$ estimates. For both approaches the virtual leak flows $\left[\mathbf{q}_{v}\right]_{i}=-\left[\mathbf{q}_{i n}\right]_{i+n_{f}}$ (the dual model's outflows to the virtual reservoirs) are used (see Figure 4, for example).

Two algorithms were used to detect leaks in the $\mathbf{q}_{v}$ : (i) the CUSUM algorithm, where a leak is detected when the cumulative sum of positive and negative differences in the signal exceeds a certain threshold $\tau_{1}$, (ii) and the likelihood ratio test (Peach et al. 1995), where a leak is detected if the ratio between the likelihood of the leak versus the leak-free case exceeds a certain threshold $\tau_{2}$. The ideal thresholds for both methods are obtained through calibration on leak free data.

Visual inspection of the virtual leakage outflows $\mathbf{q}_{v}$ of detected leaks revealed two different types of leaks. The first leak type $T_{I}$ is a sudden pipe burst that happen instantaneously at $t_{S}$

$$
q_{L}(t)=\left\{\begin{array}{l}
0 \quad \text { for } \quad t<t_{S} \\
q_{S} \text { for } t \geq t_{S}
\end{array}\right.
$$

where $q_{L}(t)$ is the leakage outflow over time and $q_{S}$ is the saturated (maximum) leak flow (e.g., Leak 3 in Figure 4). Note that leaks are not modeled as pressure dependent demands in contrast to the leaks generated in the BattLeDIM. The second leak type $T_{I I}$ is a slowly growing leak starting at $t_{S}$ and saturating at a certain time $t_{S A}$, modeled as a piecewise function with a quadratic growth rate before the saturation ((e.g., Leak 1, 2 and 4 in Figure 4).)

$$
q_{L}(t)=\left\{\begin{array}{l}
0 \text { for } t<t_{S} \\
a \cdot t^{2}+b \cdot t+c \text { for } t_{S} \leq t \leq t_{S A} \\
q_{S} \text { for } t>t_{S A}
\end{array} .\right.
$$

The coefficients of the quadratic outflow model connect the curves through following relationships $\mathrm{a}=\left(q_{S}-b\left(t_{S A}-t_{S}\right) /\left(t_{S A}{ }^{2}-t_{S}^{2}\right)\right.$ and $c=-a t_{S}^{2}-b t_{S}$. Additionally, it was found that leaks are 
evolving simultaneously in the system, which makes the detection more difficult. If a single leak evolves over time, a Bayesian inference approach based on Hamilton Monte Carlo (Hoffman and Gelman 2014) is used (e.g. in Area C) to identify the parameters $t_{S}, t_{S A}, q_{S}, a, b$, and $c$ plus the confidence intervals of the leak model parameters. In the case of multiple evolving leaks (Area $A \& B)$, differential evolution is used to identify the best combination of leak outflows over time plus the leak parameters of each single leak (Storn and Price 1997). The identified leak outflows were compared against the outcomes of the DA and subsequently used for the leak localization.

\section{Leak localization with the dual model}

The Pearson correlation for flow and pressure residuals and the first-order estimates using sensitivities are calculated for the localization (Perez et al. 2014). It is more convenient for implementation purposes to work with the pressure residuals and sensitivities of the original measurement nodes instead of using the inflow sensitivities in Eq. (14) (e.g. no need for calculating $\mathbf{A}_{f}$ and changing the set of variable pressure nodes). This does not affect the main idea, because the sensitivity of the head is equivalent to the headloss of the virtual valve and, hence, proportional to the flow sensitivity in the linearized system.

The vector of the sensitivities of measured head is determined by

$$
\nabla_{\mathbf{d}} \mathbf{h}_{\mathbf{m}}=-\mathbf{S}\left(\mathbf{A}^{T} \mathbf{F}^{-\mathbf{1}} \mathbf{A}\right)^{-1}
$$

The term $\mathbf{S}$ is the same selection matrix for the measurement nodes as in Eq. (4).

The difference between Eq. (18) and Eq. (14) consists in the multiplication by the derivative of the valve headloss: $\left([\mathbf{S h}]_{i}-h_{n_{f}+i}^{f}=K_{i}\left|\left[\mathbf{q}_{v}\right]_{i}\right|\left[\mathbf{q}_{v}\right]_{i} \Rightarrow \partial_{d_{j}}\left([\mathbf{S h}]_{i}\right)=-2 K_{i}\left|\left[\mathbf{q}_{v}\right]_{i}\right| \partial_{d_{j}}\left(\left[\mathbf{q}_{i n}\right]_{n_{f}+i}\right)\right.$ ). If the sensitivities following Eq. (18) are used, the pressure residuals are used for the calculation of the correlation, whereas the simulated external flows at the virtual reservoirs are considered in the case of Eq. (14).

It proved to be beneficial to calculate the correlations only for measurement nodes where the leak flow (calculated by the dual model) exceeds a certain threshold (e.g. $0.5 \mathrm{~L} / \mathrm{s})$. This adjustment 
eliminates the noise from the pressure measurements and stabilizes the calculated set of candidates for the unknown leak. The Pearson correlation $\rho_{\mathbf{r}, \mathbf{S}_{(\cdot \mathbf{i})}}$ is calculated as

$$
\rho_{\mathbf{r}, \mathbf{S}_{(\cdot \mathbf{i})}}=\frac{\operatorname{cov}\left(\mathbf{r}, \mathbf{S}_{(\cdot, \mathbf{i})}\right)}{\sigma_{\mathbf{r}} \cdot \sigma_{\mathbf{S}_{(, \mathbf{i})}}}
$$

where $\mathbf{r}$ is the vector of residuals, $\mathbf{S}_{(\cdot, \mathbf{i})}$ is the sensitivity vector of node $i, \operatorname{cov}($.$) is the co-variance$ and $\sigma_{\mathbf{r}}$ and $\sigma_{\mathbf{S}_{(\cdot, i)}}$ are the standard deviations of the residual vector and the sensitivity vector, respectively. The residuals and the sensitivity coefficients are very small. However, this did not show any negative impact in the allocation in our tests. In contrast, the system is stabilized by the additional pressure boundary conditions, which makes the correlation more stable compared to the conventional primal model approach. One important limitation of the correlation method is that it does not work for two or more leaks appearing at the same time. Therefore, a single leak must first be isolated in time from other leaks in order to be localized. The leakage curves that have been calculated for detection serve as a basis for choosing the best time for allocation, and we use a step by step procedure for localizing simultaneously growing leaks.

1. Identification of time interval that starts briefly before the new unknown leak starts and ends before the next leak starts. The time intervals from $t_{S}$ to $t_{S A}$ are found by a combination of CUSUM or likelihood ratio tests with Hamilton Monte Carlo or differential evolution (depending on the single or multiple leak case) as described in the leak detection paragraph in the methods section.

2. Initialize calculation for the selected time interval (load all measurements as well as the estimated demands)

3. Run Extended Period Simulations for selected time interval; for each time step do:

(a) Update boundary conditions via toolkit functions including demand patterns, heads at virtual reservoirs, pump flow.

(b) Update all known leaks with their calculated leak flows as fixed demands and define the 
start time of the unknown leak based on the results of the detection.

(c) Simulation of the time step (here the EPANET toolkit is used) and after each time step with active new unknown leak, calculate correlation in Eq. (19) for all nodes based on the sensitivities.

(d) Consider only the nodes with a correlation score higher than a given minimum threshold (e.g. 0.95) and add those eligible correlations to the sum of correlation taken over all calculated time steps.

4. The node with the highest correlation sum is identified as the candidate for the new leak within this time interval.

5. The new leak is added to the list of known leaks and the leakage flow is considered as known demand for the localization of the next leak and the procedure is repeated from point 1 until all leaks have been identified in the given period.

\section{L-Town case study and measurement data}

The case study network L-Town was provided by the organizers of the BattLeDIM (Vrachimis et al. 2020). L-Town is a small hypothetical town based on a real WDN in Cyprus with approximately 10,000 inhabitants, which receive water from two reservoirs. The WDN consists of pipes with diameters ranging from $63 \mathrm{~mm}$ to $225 \mathrm{~mm}$ and a total pipe length of $43 \mathrm{~km}$. L-Town consists of three distinct hydraulic areas: (i) Area A is the main part of the network, (ii) Area B is a low lying part that is supplied through a pressure reduction valve, and (iii) Area $\mathrm{C}$ is an area with higher elevation that is supplied by an elevated tank fed from Area A through a pumping station. An overview of the network and the location of the three measurement zones can be found in Figure 2.

To enhance the water loss monitoring capabilities, the WU of L-Town installed three flow meters (two at the reservoirs and one at the pumping station), a tank level sensor and 33 pressure sensors (depicted as circles in Figure 2). All sensors measure and transmit data every 5 minutes to the utility's supervisory control and data acquisition (SCADA) system. Additionally, the WU installed 82 smart water meters or AMRs in Area C, measuring three different customer types: residential, 
commercial and industrial. There is no flow meter installed at the tank that feeds Area C. Therefore, a virtual inflow measurement to Area $\mathrm{C}$ has to be reconstructed from the tank level measurements and the inflow measurement measured at the pump that supplies the tank.

The dataset of the BattLeDIM contains two years of sensor data for years 2018 (historical dataset) and 2019 (validation dataset), an EPANET model of the water distribution network, plus the time and repair location of ten pipe bursts that have been fixed in 2018. Three types of leaks exist: (i) small background leaks with $1 \%-5 \%$ of the average inflow, (ii) medium pipe breaks with $5 \%-10 \%$, and (iii) large pipe bursts with leakage flows of more than $10 \%$ of the average system inflow $\left(\approx 180 \mathrm{~m}^{3} / \mathrm{h}\right)$. Large leakages with outflows over $15 \mathrm{~m}^{3} / \mathrm{h}$ are fixed by the water utility after a reasonable amount of time within two months. The leakages have two different time profiles, (i) either abrupt pipe bursts with constant leak flow rates, (ii) or background leakages with growing leak rates which evolve over time until large outflow rates at which they remain constant. In total, 14 leakages occurred in 2018 with outflow rates between 5 to $35 \mathrm{~m}^{3} / \mathrm{h}$, of which 10 leaks have been repaired. The remaining 4 leaks are not repaired and continue into the 2019 validation dataset. The BattLeDIM challenge is to find the 19 leaks that happened in 2019 plus the 4 remaining leaks. The outflows and locations of the 33 leaks can be found in Figures 7 to 10 (dashed lines in the outflow time series plots and circles in the location overview plots). More details on the dataset can be found in (Vrachimis et al. 2020).

\section{RESULTS AND DISCUSSION}

\section{Demand calibration}

Each AMR time series is decomposed into its trend, seasonal (with a period length of a week), and random components using the multiplicative time series model described in Eq. (1). Subsequently, cluster analysis is used to identify similarities in the trend and seasonal patterns. Two distinct demand patterns emerge in the trend $T(t)$ and in the seasonal components $S(t)$, a residential $\left(T_{\mathrm{R}}(t), S_{\mathrm{R}}(t)\right)$ and a commercial $\left(T_{\mathrm{C}}(t), S_{\mathrm{C}}(t)\right)$ one. The seasonal and the trend components are shown in Figure 3 for each AMR measurement. Furthermore, some patterns are found to be a superposition of both pattern types. These patterns belong to houses with mixed user groups (e.g. 
commercial space in the ground floor and apartments in the floors above). Subsequently, these patterns are called mixed patterns. Generally, all demand patterns can be described through the superposition (see Eq.(2)) of the residential and the commercial pattern. During workdays (Monday to Friday), water consumption follows a similar behavior, whereas during the weekend (Saturday and Sunday) higher consumption during late hours occur as the result of night life (see Figure 3 (a)). Furthermore, cluster analysis revealed four outlier pattern in the AMR measurements. After closer examination, these outlier patterns were explained as industrial users with a periodicity differing from a week (i.e. 9, 11 or 13 days). Hence, those industrial users do not follow the same pattern of consumption as described in Eq. (2) and are not further used in the demand modeling. The trend components in Figure 3 (b) show higher water usage during July/August, and lower in December/January.

The demand model is used to model the unmeasured customers within the L-town network. Additionally, a virtual inflow measurement of Area $\mathrm{C}$ has been constructed from the pump flow measurements and the tank's water level. This virtual inflow is used to (i) validate the demand model and to (ii) estimate the leak outflow in Area C. Figure 4 (a) shows the estimated leakage outflow, which is constructed as the difference between the virtual inflow measurement and the total estimated demand for Area C. Three different strategies for the demand estimation are used in Area C. First, only the measured demand at the AMRs is subtracted (just AMR in Figure 4 (a)), which leads to an overestimation or an offset of the leak flow, because of the unmeasured customers. Second, the demand for the whole zone is estimated based on the model as described in Eq. 2 using the base demands from the BattLeDIM EPANET model (Inferred), which leads to a high noise in the leak outflow estimates. Third, the AMR measurements are combined with demand estimates for the unmeasured customers (Combined). The last approach leads to the best leak outflow estimates with low levels of noise as well as no offset. Clearly, four different leaks can be seen in the data, three are growing over time until they are saturated (Leak 1, 2, and 4), and a sudden pipe burst (Leak 3). This information proved to be useful for the leakage modeling (see Eq. (16) and (17)). 


\section{Roughness calibration}

The internal diameters of pipes are nominal diameters defined by a discrete number of values that depend on the manufacturer and the material. In the L-Town INP file, it is assumed that the outside diameters of plastic pipes are entered instead of the inside diameters, which is first corrected with the most usual inside diameter for PVC and PE pipes (see Table 1).

After inspection and several tests, the pipes are divided in six different roughness clusters according to their diameter, material, initial roughness values and managing zones in which they are located : Because of the small number of observations and pipes, one cluster with $C_{H W}=x_{5}$ is assigned for Zone $\mathrm{B}$ and one to Zone $\mathrm{C}\left(x_{6}\right)$. Cluster with same $x_{1}$ roughness value consists of the plastic pipes in Zone A; pipes in cluster 2 are in Zone A with diameters $100 \mathrm{~mm}$ or $150 \mathrm{~mm}$, and original INP roughness $x_{2}=120$. Similarly, pipes in zone A with diameters $100 \mathrm{~mm}$ or $150 \mathrm{~mm}$ and original $C_{H W}=140$ define the cluster 3: $x_{3}=140$. Finally, cluster 4 is made of pipes with internal diameter $200 \mathrm{~mm}$ in Zone A. Figure 2 shows an overview of the roughness groups. Through visual inspection of the measurements from the first week of 2018, it is assumed that no leaks are present in the dataset during that time. Consequently, measurements for this week are used for the roughness calibration. The roughness calibration is performed for the six clusters, $n_{c}=6$, and by solving the WLS problem in Eq. (5) with $\kappa=3, \alpha=0$ and box constraints $x^{L}=60$ and $x^{U}=160$ with the Levenberg-Marquardt method (10). The $n_{s}=33$ pressure measurements in Figure 2 are used $\left(n_{m}=33\right)$. They repeat every five minutes for 7 days $\left(n_{t}=2016\right)$. All measurements are chosen to be of the same accuracy $\sigma_{i j}=1$.

The algorithm converges after 11 iterations. The results are given in terms of estimates in Table 2. For the first cluster, plastic pipes in Zone A, the initial estimate $x_{1}^{0}=146$ belongs to the $99 \%$ confidence interval $[141.9,163.7]$. Consequently, the final estimate 152.8 is not significantly different from the initial value. However, the initial estimates for the other five clusters differ significantly from the point estimates at iteration $k=11$ (they do not belong to the five $99 \%$ confidence intervals). Based on the confidence intervals and the initial estimates, the bold values are selected. The pressure residuals are represented in Figure 2. It can be seen that the mean 
squared error (MSE) is about $6 \mathrm{~cm} \mathrm{H}_{2} \mathrm{O}$.

\section{Virtual leak flows with the dual model}

A dual model is constructed from the EPANET model containing the calibrated pipe roughnesses and demand patterns from the demand calibration. The heads of the virtual reservoirs are set to the corresponding pressure measurements. If leaks appear in the network, the dual model reacts with virtual leak outflows caused by the pressure differences of the hydraulic model and the lower reservoir heads. The virtual leak flows for each sensor location within Area $\mathrm{C}$ are depicted in Figure 4 (b). Furthermore, the total sum of all virtual leak flows is shown. This sum gives a good first approximation of the leak size. The second leak in Area $\mathrm{C}$ was repaired and, hence, its end time and its location (pipe p31) are known. The leak is closest to sensor node n31, which shows the strongest reaction to the leak by producing the biggest virtual outflow. Same reasoning leads to the conclusion that Leak 1 is close to sensor $n 1$, Leak 3 is in proximity of $n 31$, and Leak 4 is somewhere in the middle of all three sensors.

Comparison of Figure 4 (a) with the total virtual leak outflow in (b) shows that the real leakage outflows have similar magnitudes as the virtual outflows. However, the dual model seems to underestimate the real outflows in Area C slightly.

Figure 5 shows an comparison of the effect of leakages on the measured pressure signals versus the virtual leak flows in the dual model for the first two leakages in 2019 that appear in Area A (pipe p523 and p810). In this Figure, solid lines are four hour moving averages, whereas the shaded lines are the original five minutes signal. The dual model amplifies the leak signal compared to the pressures (compare Figure 5 (a) and (b)). Furthermore, the leaks have a more local effect on the virtual leak flows than in the pressures, which allows already a rough estimation of the leak's location. The sum of all virtual leak outflows in Figure 5 (c) gives already a good estimate of the leak sizes, which are approximately $27 \mathrm{~m}^{3} / \mathrm{h}$ for each leak.

\section{Leak Detection}

Two different signals are used for leak detection; (i) the flow residual between the measured inflow and total demand plus already known leaks in an area, (ii) the dual model's outflows to the 
virtual reservoirs (see Figure 4 or Figure 5). Two different types of leaks are found in the data instant bursts and leaks that are growing over time. Growing leakage flows are modeled with the quadratic function in Eq. (17). Data from the dual model is used to identify the leak start times and their shapes (i.e. instant or growing). For that reason, thresholds are extracted from the DA flows at each sensor using the leak free case in the first week of 2018. If the DA signal exceeds the threshold, a leak is detected in the system. The detection time is used as the start time of the leak for our BattLeDIM solution. To estimate the leakage outflow, the start times and the shapes of the leaks are used to fit the leak shape on the flow residuals. If a single leak evolves over time, Bayesian inference is used, for multiple simultaneously appearing leaks, a faster differential evolution is used to identify the best combination of leak outflows over time. The detected leaks are double checked against the DA and subsequently used for the leak localization.

The results for leak detection and localization for 2019 are summarized in Table 3. Additionally, the leak detection and localization results are broken down by the different areas are shown in Figures 7 to 10, where shaded lines are daily moving averages of the real leakages, solid dashed lines are the estimated leakages. Circles in the network maps are the real leak locations, while crosses show our estimates. The leak detection results for Area $\mathrm{C}$ are shown in Figure 7 (a). The shapes of the leaks are resembled very well by our method for all three leaks, and the differences in the final leak outflows are negligible for Area C. The sudden pipe burst (Leak C3 at pipe p280) is detected instantaneously, while the detection of the growing leaks takes a bit longer. Nevertheless, leakages are detected on average within less than 9 days. A less conservative detection threshold potentially decreases the detection time.

The leak detection results for Area B are shown in Figure 8 (a), where the instant pipe burst is perfectly detected, although the leakage outflow is slightly overestimated.

The leak detection results for Area A are shown in Figure 9. For a better visibility of the simultaneously appearing leaks, the Figure is split into the two half-years of 2019, with (a) for the first half until July, and (b) showing the second half of the year. Additionally, the leaks from the first half are depicted as gray shaded lines in Figure 9 (b) as they are still present in the network. Sudden 
pipe bursts are again detected instantaneously, while the thresholds for growing leaks seemed a bit too conservative. However, the shapes of all leaks are very well described through the coefficients that our model found. One leak that started in February 2018 at pipe p427 with a magnitude of $5 m^{3} / h$ is not detected at all. All leak shapes are identified correctly until August, when Leak A17 at pipe p721 appears (see Figure 9 (b)). However, this leak is detected very late and its size is underestimated by almost $5 \mathrm{~m}^{3} / \mathrm{h}$. This influences the detection of subsequent leaks, which results in a decrease in the detection as well as the localization performance. Nevertheless, leakages in Zone A were detected within 10 days on average.

\section{Leak Localization}

For the localization of the leaks the network is divided into two separate parts $(A+B$ and $C)$ and the pump is replaced by the flow measurement for Zone A and B. All calculations are executed by use of EPANET 2.00.12 (Rossman 2000) and the EPANET toolkit integrated in an application for data management and visualization that is exclusively developed for the performance of the project.

Figure 6 visualizes the GUI-output at a certain time step. The circles indicate the locations of the pressure measurement nodes and the numbers show the calculated in- and outflows calculated by the dual model. The two biggest virtual reservoirs outflows are marked by a bigger circle as expected in the neighborhood of these two nodes. The diamonds show the nodes with highest correlation scores at the current time and the bigger diamonds show the nodes with highest correlation sum. Their size is scaled by the sum value which means that they are growing over time.

Figure 7 (b) shows the localization results for Area C. Leak $\mathrm{C} 1$ is perfectly isolated at the real location (p257). Leak C3 is found within $50 \mathrm{~m}$ of the real leak. However, if the closed valve in Area $\mathrm{C}$ is added to the hydraulic model, the isolation of this leak might improve further. Leak $\mathrm{C} 4$ is not localized correctly, since the distance exceeds $300 \mathrm{~m}$ as stated in the BattLeDIM rules. Reasons for that might be that the closed valve is not taken into account, or the fact that we are using demand driven models, while the BattLeDIM organizers used a pressure-driven model. The more leakages occur in the network, the greater the difference between a demand-driven and a pressure-driven demand model become, and the more inexact our localization gets, since the localization errors 
accumulate. On average, all leaks are found within $130 \mathrm{~m}$ of the real leak in Area C. For Area B, the leak is perfectly isolated in time as well as in space (see Figure 8).

The results for Area A can be found in Figure 10, and are split again into half-years. Figure 10 (a) also contains the leak that was not detected by our method (white cross). Early leaks are isolated almost perfectly, while the localization gets worse during later simulations. This might be a consequence of the demand-driven model that is used. For the leaks in Figure 10 (a), the average distance of the real leaks to the estimated leak position is around $150 \mathrm{~m}$. During later simulations, this distance increases to $250 \mathrm{~m}$ (see Figure 10 (b) and Table 3). It has to be noted that a typo occurred while submitting the results for the BattLeDIM. Leak p654 was inserted as p645. Taken this into account, the final score of the Team Under Pressure would even further increase from already the highest rate of true positives of $65 \%$ of all participating teams.

\section{CONCLUSION}

In this work, we present a novel solution to detect and isolate multiple-leaks in WDN that we developed while participating in the BattLeDIM competition. Our method consists of calibrating the nodal demand and pipe roughness, and introducing a dual model for the calibrated primal problem to detect and locate leaks.

The calibration uses time series analysis and cluster analysis to build a multiplicative predictive model for ultimately two network-wide demand models, a residential and a commercial model. This is used for both, (i) modeling unknown demands over time in the hydraulic model, as well as distinguishing leakages and consumption in the measurements. Subsequently, six roughness clusters were calibrated using 33 pressure loggers for the first week of 2018. Confidence intervals are given for the least-squares estimates. The pressure residuals are very well reproduced for the entire week with a small root mean square error of $6 \mathrm{~cm}$.

The core of our method is a dual model that transforms a pressure measurement node into a free junction node plus a link to a virtual reservoir, whose head is equal to the measured values. Significant inflows or outflows, either sudden or gradual, to these virtual reservoirs are indications of leaks. In the dual model, the pressure signal is transformed into a virtual leakage outflow 
signal that is easier to analyze since it amplifies and localizes the effects of leaks in the network. Sensitivities of nodal pressures to virtual outflows are also derived. They are essential to understand the behavior of the model at first order.

For leak detection, the CUSUM algorithm and likelihood-ratio tests are used jointly on the virtual leak flows, where the parameters are tuned to limit the global false positive rate under normal operation conditions. When multiple leaks are present, differential evolution is used to identify the best combination of leak modeling parameters over time (i.e. start times and shapes of leaks over time). These detection methods were employed for both, the primal and the dual data. The localization is achieved by analyzing the correlation between the calibrated pressure (or virtual inlet-outlet model predictions) and the corresponding first-order leakage impulse response predictions at the candidate nodes. This solution recovered $65 \%$ of true leaks with only four false positives in all of 2019, which is a notable result (shared \#1 ranking).

Using a pressure-driven model instead of a demand-driven one, improving the calibration by reliably detecting closed valves, as well as using less conservative threshold parameters for the detection of the growing leaks might increase the already notable result further. Certainly, a lot of potential lies in a deeper understanding of the dual model to further improve the detection and isolation of multiple simultaneously occurring leaks.

With 33 pressure sensors, the BattLeDIM dataset contains an unrealistic high number of sensors in a WDN of that size. Indeed, the leak detection and localization performance will decrease with a lower number of sensors. However, optimal sensor placement algorithms might recover similar leak detection and localization performances with fewer sensors. Furthermore, the BattLeDIM organizers constructed the nodal demand patterns through a superposition of residential and commercial demands multiplied with noise. That is why we were able to almost precisely reconstruct the demands on the unmeasured locations through the information contained in the AMR data with our demand calibration approach. In reality, demand patterns are more variable (Steffelbauer et al. 2021). Consequently, the dual model might perform worse in systems with limited demand information and, hence, less accurate demand models. 
That is why for future work, we want to focus on optimal sensor placement (Steffelbauer and Fuchs-Hanusch 2016a) with the dual model and on applying the dual model on challenging real data sets, with model errors, outliers, uncertainty, and more variable and realistic water demands. Furthermore, we are planning to investigate the importance of each step for the final classification, enhancing the method to reduce the false positive rate, and study the effect of the dual model on fitness landscapes of WDN optimization problems (Steffelbauer and Fuchs-Hanusch 2016b).

\section{DATA AVAILABILITY STATEMENT}

All data, models, or code that support the findings of this study are available from the corresponding author upon reasonable request.

\section{ACKNOWLEDGMENTS}

This project has received funding from the European Union's Horizon 2020 research and innovation programme under the Marie Skłodowska-Curie grant agreement No 707404. The opinions expressed in this document reflect only the author's view. The European Commission is not responsible for any use that may be made of the information it contains. One author of this paper was supported in part by the German Ministry for Education and Research (BMBF Project W-Net 4.0 02WIK1477C). 


\section{APPENDIX}

AMR automatic meter reading

BattLeDIM Battle of the Leak Detection and Isolation Methods

CUSUM cumulative sum control chart

DA Dual Approach

DMA district metered area

HW Hazen-Williams

MSE mean squared error

SCADA supervisory control and data acquisition

TSA time series analysis

WLS weighted least squares

WDN water distribution network

WU water utility

\section{REFERENCES}

Adedeji, K. B., Hamam, Y., Abe, B. T., and Abu-Mahfouz, A. M. (2017). "Towards Achieving a Reliable Leakage Detection and Localization Algorithm for Application in Water Piping Networks: An Overview." IEEE Access, 5, 20272-20285.

Bakker, M., Vreeburg, J. H. G., Van De Roer, M., and Rietveld, L. C. (2014). "Heuristic burst detection method using flow and pressure measurements." Journal of Hydroinformatics, 16(5), 1194-1209.

Farley, M. and Trow, S. (2003). Losses in water distribution networks: A practitioner's guide to assessment. IWA publishing.

Goulet, J.-A., Coutu, S., and Smith, I. F. (2013). "Model falsification diagnosis and sensor placement for leak detection in pressurized pipe networks." Advanced Engineering Informatics, 27(2), 261269. 
Gupta, A. and Kulat, K. D. (2018). "A selective literature review on leak management techniques for water distribution system.” Water Resources Management, 32(10), 3247-3269.

Hamilton, S. and McKenzie, R. (2014). Water management and water loss. IWA Publishing.

Hoffman, M. D. and Gelman, A. (2014). “The no-U-turn sampler: Adaptively setting path lengths in Hamiltonian Monte Carlo.” Journal of Machine Learning Research.

Hu, Z., Chen, B., Chen, W., Tan, D., and Shen, D. (2021). "Review of model-based and data-driven approaches for leak detection and location in water distribution systems." Water Supply.

Hutton, C. J., Kapelan, Z., Vamvakeridou-Lyroudia, L., and Savić, D. A. (2014). "Dealing with Uncertainty in Water Distribution System Models: A Framework for Real-Time Modeling and Data Assimilation.” Journal of Water Resources Planning and Management, 140(2), 169-183.

Moser, G., Paal, S. G., and Smith, I. F. (2017). "Measurement system design for leak detection in hydraulic pressurized networks." Structure and Infrastructure Engineering, 13(7), 918-928.

OECD (2016). Water Governance in Cities. OECD Publishing, Paris, <https://www.oecdilibrary.org/content/publication/9789264251090-en>.

Peach, N., Basseville, M., and Nikiforov, I. V. (1995). "Detection of Abrupt Changes: Theory and Applications.." Journal of the Royal Statistical Society. Series A (Statistics in Society), 158(1).

Pérez, R., Puig, V., Pascual, J., Quevedo, J., Landeros, E., and Peralta, A. (2011). "Methodology for leakage isolation using pressure sensitivity analysis in water distribution networks." Control Engineering Practice, 19(10), 1157-1167.

Perez, R., Sanz, G., Puig, V., Quevedo, J., Cuguero Escofet, M. A., Nejjari, F., Meseguer, J., Cembrano, G., Mirats Tur, J. M., and Sarrate, R. (2014). "Leak localization in water networks: A model-based methodology using pressure sensors applied to a real network in barcelona [applications of control]." IEEE Control Systems.

Piller, O. (2019). “Water distribution system modeling and optimization.” French habilitation, Ecole doctorale Sciences des métiers de l'ingénieur, ED 432, Paris, France.

Piller, O., Elhay, S., Deuerlein, J., and Simpson, A. R. (2017). "Local sensitivity of pressuredriven modeling and demand-driven modeling steady-state solutions to variations in parameters." 
journal of Water Resources Planning and Management, 143(2).

Preis, A., Whittle, A. J., Ostfeld, A., and Perelman, L. (2011). "Efficient hydraulic state estimation technique using reduced models of urban water networks." journal of Water Resources Planning and Management, 137(4), 343-351.

Pudar, R. S. and Liggett, J. A. (1992). "Leaks in pipe networks.” journal of Hydraulic Engineering, $118(7), 1031-1046$.

Puust, R., Kapelan, Z., Savic, D. A., and Koppel, T. (2010). “A review of methods for leakage management in pipe networks." Urban Water journal, 7(1), 25-45.

Romano, M., Kapelan, Z., and Savić, D. A. (2013). "Geostatistical techniques for approximate location of pipe burst events in water distribution systems." Journal of Hydroinformatics, 15(3).

Rossman, L. A. (2000). Epanet 2 User 's Manual. US EPA.

Sanz, G., Pérez, R., Kapelan, Z., and Savic, D. (2016). "Leak detection and localization through demand components calibration." journal of Water Resources Planning and Management, 142(2), 04015057.

Savic, D. A., Kapelan, Z. S., and Jonkergouw, P. M. (2009). “Quo vadis water distribution model calibration?." Urban Water Journal.

Seabold, S. and Perktold, J. (2010). "Statsmodels: Econometric and statistical modeling with python." 9th Python in Science Conference.

Shumway, R. H. and Stoffer, D. S. (2010). Time Series Analysis and Its Applications With R Examples. Springer International Publishing, 4 edition.

Soldevila, A., Blesa, J., Tornil-Sin, S., Duviella, E., Fernandez-Canti, R. M., and Puig, V. (2016). "Leak localization in water distribution networks using a mixed model-based/data-driven approach.” Control Engineering Practice, 55, 162-173.

Soldevila, A., Fernandez-Canti, R. M., Blesa, J., Tornil-Sin, S., and Puig, V. (2017). "Leak localization in water distribution networks using bayesian classifiers." Journal of Process Control, $55,1-9$.

Steffelbauer, D. B., Blokker, E. J. M., Buchberger, S. G., Knobbe, A., and Abraham, E. (2021). 
"Dynamic time warping clustering to discover socioeconomic characteristics in smart water meter data." journal of Water Resources Planning and Management, 147(6), 04021026.

Steffelbauer, D. B., Deuerlein, J., Gilbert, D., Piller, O., and Abraham, E. (2020). “A Dual Model For Leak Detection and Localization." BattLeDIM: Battle of the Leakage Detection and Isolation Methods, Vrachimis, Eliades, Taormina, Ostfeld, Kapelan, Liu, Kyriakou, Pavlou, Qiu, and Polycarppou, eds., Beijing, China, Zenodo, <https://doi.org/10.5281/zenodo.3923907>.

Steffelbauer, D. B. and Fuchs-Hanusch, D. (2016a). "Efficient Sensor Placement for Leak Localization Considering Uncertainties.” Water Resources Management, 30(14), 5517-5533.

Steffelbauer, D. B. and Fuchs-Hanusch, D. (2016b). "Fitness landscapes and distance metrics for model-based leakage localization." 2016 3rd Conference on Control and Fault-Tolerant Systems (SysTol), IEEE, 343-348, <http://ieeexplore.ieee.org/document/7739774/>.

Storn, R. and Price, K. (1997). "Differential Evolution - A Simple and Efficient Heuristic for Global Optimization over Continuous Spaces." Journal of Global Optimization.

Vrachimis, S. G., Eliades, D. G., Taormina, R., Ostfeld, A., Kapelan, Z., Liu, S., Kyriakou, M. S., Pavlou, P., Qiu, M., and Polycarpou, M. (2020). "Dataset of BattLeDIM: Battle of the Leakage Detection and Isolation Methods." dataset, <https://doi.org/10.5281/zenodo.4017659> (September).

Wu, Z. Y. (2008). "Innovative Optimization Model for Water Distribution Leakage Detection." Bentley Methods Solution Center, 1-8. 


\section{List of Tables}

1 Original pipe characteristics in the INP file and corresponding cluster membership; in red the original external parameters that were corrected for PVC and PE pipes. . 30

2 Calibration of HW coefficients; the first three columns are the lower bound, initial estimate, and upper bound values for the six clusters; the last three columns are the $99 \%$ confidence intervals centered on the value at convergence; in bold the final point estimate. . . . . . . . . . . . . . . . . 31

3 Results of leak detection and localization: The true location, the start time and the maximum leakage outflow $\max \left(Q_{L}\right)$ are taken from the BattLeDIM solutions. The estimated location is found with the leak localization algorithm. $t_{D}$ is the detection time measured in hours since the true start time of the leak. The distance between the true and the estimated leak location is the shortest topological distance over the pipes in meter. Zone shows in which area of the network the leak is located. Perfectly located leaks are shown in boldface (plus minus $10 \mathrm{~m}$ ), while leaks with a distance greater than $300 \mathrm{~m}$ (missed leaks according to the BattLeDIM rules) are highlighted with an asterisk. . . . . . . . . . . . . . . 32 
TABLE 1. Original pipe characteristics in the INP file and corresponding cluster membership; in red the original external parameters that were corrected for PVC and PE pipes.

\begin{tabular}{l|c|c|c|c|r}
\hline $\begin{array}{l}\text { Diameter } \\
\text { in mm }\end{array}$ & C $_{H W}$ & Zone & $\begin{array}{c}\text { Cluster } \sharp \\
\text { in Eq. (3) }\end{array}$ & $\sharp$ pipes & $\begin{array}{r}\text { Length } \\
\text { in m }\end{array}$ \\
\hline $53.6(63)$ & 146 & $\mathrm{~A}$ & 1 & 3 & 71.40 \\
$53.6(63)$ & 146 & $\mathrm{~B}$ & 5 & 1 & 9.21 \\
$64(75)$ & 146 & $\mathrm{~A}$ & 1 & 1 & 60.08 \\
100 & 120 & $\mathrm{~A}$ & 2 & 76 & 3639.10 \\
100 & 120 & $\mathrm{~B}$ & 5 & 25 & 1190.11 \\
100 & 140 & $\mathrm{~A}$ & 3 & 500 & 24069.65 \\
100 & 140 & $\mathrm{C}$ & 6 & 104 & 5201.60 \\
150 & 120 & $\mathrm{~A}$ & 2 & 7 & 313.62 \\
150 & 140 & $\mathrm{~A}$ & 3 & 90 & 4102.87 \\
150 & 120 & $\mathrm{~B}$ & 5 & 6 & 226.56 \\
$141(160)$ & 146 & $\mathrm{~A}$ & 1 & 16 & 713.73 \\
200 & 90 & $\mathrm{~A}$ & 4 & 59 & 2749.71 \\
200 & 90 & $\mathrm{C}$ & 6 & 5 & 195.90 \\
$198.2(225)$ & 146 & $\mathrm{~A}$ & 1 & 12 & 558.58 \\
\hline
\end{tabular}


TABLE 2. Calibration of HW coefficients; the first three columns are the lower bound, initial estimate, and upper bound values for the six clusters; the last three columns are the $99 \%$ confidence intervals centered on the value at convergence; in bold the final point estimate.

\begin{tabular}{c|c|c|c|c|c|c}
\hline Cluster $\sharp$ & $\mathbf{x}^{L}$ & $\mathbf{x}^{0}$ & $\mathbf{x}^{U}$ & $\mathbf{x}^{11}-\Delta_{x}$ & $\mathbf{x}^{11}$ & $\mathbf{x}^{11}+\Delta_{x}$ \\
\hline 1 & 60 & $\mathbf{1 4 6}$ & 160 & 141.9 & 152.8 & 163.7 \\
2 & 60 & 120 & 160 & 108.1 & $\mathbf{1 0 9 . 7}$ & 111.3 \\
3 & 60 & 140 & 160 & 141.1 & $\mathbf{1 4 1 . 6}$ & 142.1 \\
4 & 60 & 90 & 160 & 126.5 & $\mathbf{1 2 6 . 8}$ & 127.1 \\
5 & 60 & 136 & 160 & 100.4 & $\mathbf{1 1 1 . 2}$ & 122.0 \\
6 & 60 & 133 & 160 & 133.1 & $\mathbf{1 3 4 . 0}$ & 134.9 \\
\hline
\end{tabular}


TABLE 3. Results of leak detection and localization: The true location, the start time and the maximum leakage outflow $\max \left(Q_{L}\right)$ are taken from the BattLeDIM solutions. The estimated location is found with the leak localization algorithm. $t_{D}$ is the detection time measured in hours since the true start time of the leak. The distance between the true and the estimated leak location is the shortest topological distance over the pipes in meter. Zone shows in which area of the network the leak is located. Perfectly located leaks are shown in boldface (plus minus $10 \mathrm{~m}$ ), while leaks with a distance greater than $300 \mathrm{~m}$ (missed leaks according to the BattLeDIM rules) are highlighted with an asterisk.

\begin{tabular}{|c|c|c|c|c|c|c|}
\hline $\begin{array}{l}\text { True Loc. } \\
\text { - }\end{array}$ & $\begin{array}{c}\text { start time } \\
-\end{array}$ & $\begin{array}{r}\max \left(Q_{L}\right) \\
\left(m^{3} / h\right)\end{array}$ & $\begin{array}{l}\text { Est. Loc. } \\
-\end{array}$ & $\begin{array}{r}t_{D} \\
(h)\end{array}$ & $\begin{array}{r}\text { Distance } \\
(m)\end{array}$ & $\begin{array}{c}\text { Zone } \\
-\end{array}$ \\
\hline p427 & 2018-02-13 08:25 & 5.11 & - & - & - & A \\
\hline p654 & 2018-07-05 03:40 & 5.49 & p654 & 956.33 & 0 & A \\
\hline p810 & 2018-07-28 03:05 & 6.91 & p810 & 668.92 & 0 & A \\
\hline p523 & 2019-01-15 23:00 & 28.39 & p500 & 0.00 & 205 & A \\
\hline p827 & 2019-01-24 18:30 & 26.46 & p827 & -0.08 & 0 & A \\
\hline p653 & 2019-03-03 13:10 & 18.28 & p655 & 273.42 & 106 & A \\
\hline p710 & 2019-03-24 14:15 & 5.58 & p702 & 0.00 & 222 & A \\
\hline p514 & 2019-04-02 20:40 & 15.58 & p226 & 0.00 & 90 & A \\
\hline $\mathrm{p} 331^{(*)}$ & 2019-04-20 10:10 & 10.93 & p905 & 0.00 & 355 & A \\
\hline p193 ${ }^{(*)}$ & 2019-05-19 10:40 & 10.36 & p185 & 417.33 & 398 & A \\
\hline p142 & 2019-06-12 19:55 & 27.04 & p623 & 0.00 & 2 & A \\
\hline p586 & 2019-07-26 14:40 & 20.52 & p586 & 215.50 & 0 & A \\
\hline p721 ${ }^{(*)}$ & 2019-08-02 03:00 & 13.18 & p703 & 222.92 & 354 & A \\
\hline p800 & 2019-08-16 14:00 & 21.95 & p820 & 110.50 & 196 & A \\
\hline p123 & 2019-09-13 20:05 & 9.19 & p201 & 588.33 & 133 & A \\
\hline p455 & 2019-10-03 14:00 & 11.05 & p109 & 584.92 & 142 & A \\
\hline p762 & 2019-10-09 10:15 & 15.71 & p745 & 301.00 & 179 & A \\
\hline $\mathrm{p} 426^{(*)}$ & 2019-10-25 13:25 & 13.56 & p42 & 0.00 & 779 & A \\
\hline p879 & 2019-11-20 11:55 & 10.93 & p884 & 342.50 & 256 & A \\
\hline p680 & 2019-07-10 08:45 & 5.37 & p680 & 0.00 & 0 & B \\
\hline p257 & 2018-01-08 13:30 & 6.87 & p257 & 104.50 & 0 & $\mathrm{C}$ \\
\hline p280 & 2019-02-10 13:05 & 5.26 & p251 & 0.00 & 49 & $\mathrm{C}$ \\
\hline $\mathrm{p} 277^{(*)}$ & 2019-05-30 21:55 & 7.36 & $\mathrm{p} 8$ & 541.83 & 358 & $\mathrm{C}$ \\
\hline
\end{tabular}




\section{List of Figures}

1 Overview of the hierarchical leak detection and isolation approach from left to right: Starting with the data analysis (measurements and EPANET model), then model calibration (nodal demand and pipe roughness), followed by simulations with the dual model approach, to finally detect and localize leaks. . . . . . . . . . . 35

2 Network colored by calibration clusters of Hazen-Williams roughness coefficients. Pressure measurements are shown as circles. In a) the roughness iterations are plotted ; in b), the inset shows the principle of the dual model, where the pressure measurements are replaced by the combination of a valve and a virtual reservoir whose head is equal to the measured head $h_{i}$; the dual model transforms $h_{i}$ into virtual leakage flows $q_{v_{i}}$; in (c) the pressure residuals are shown for the first week of 2018; and finally, in (d) the minimum, maximum, and root mean square errors (RMSE) are shown in increasing RMSE order. . . . . . . . . . . . . . . . 36

3 Weekly seasonality (a) and yearly trend (b) extracted from the AMR measurements for the different customer types (Residential and Commercial) and nodes consisting of a mix of them (Mixed). . . . . . . . . . . . . . 37

4 Leakage outflow in Area C (a) estimated by comparing the "virtual" inflow measurement and the demand model and (b) as provided by the dual model. . . . . . . 38

5 Dual model signals for first two leaks in Area A in 2019 (location at pipes p827 and p523 with magnitudes of approximately $27 \mathrm{~m}^{3} / \mathrm{h}$ each). (a) Pressure measurements $p$ over time, (b) sharp and localized signal of the virtual leak outflows $q_{v}$ over time calculated by the dual model at the same measurement locations, (c) the sum over all virtual leak flows in the dual model serves as good estimates for leak size. . . . 39

6 Snapshot of the leakage isolation tool: calculated outflows at virtual reservoirs of sensor nodes and correlation results: small diamonds for current time step and large diamonds for sum of all time steps (the size represents the score) . . . . . . . . . . 40 
7 Results of leak detection and localization for the unknown leaks in Area C in 2019:

(a) Identified leakage outflows over time and (b) estimated locations of the leaks. Crosses are the estimated leak locations, circles indicate the real locations. . . . . . 41

8 Results of leak detection and localization for the unknown leaks in Area B in 2019:

(a) Identified leakage outflows over time; and (b) estimated locations of the leaks. The Cross is the estimated leak location, the circle indicates the real location. . . . 42

9 Results of leak detection for the unknown leaks in Area A in 2019: (a) Leakage outflows for the first half of the year / leaks, and (b) for the second half of the year / leaks. The second half also includes the ongoing leaks from (a) as shaded lines. Additionally, the missed detected leak at pipe p427 is shown in (a) . . . . . . . . . 43

10 Results of leak localization for the unknown leaks in Area A in 2019: (a) First half of the year from January to June, and (b) for the second half of the year from July to December. The not detected leak at pipe 427 is shown as a white cross in (a). Crosses are the estimated leak locations, circles indicate the real locations. . . . . . 44 


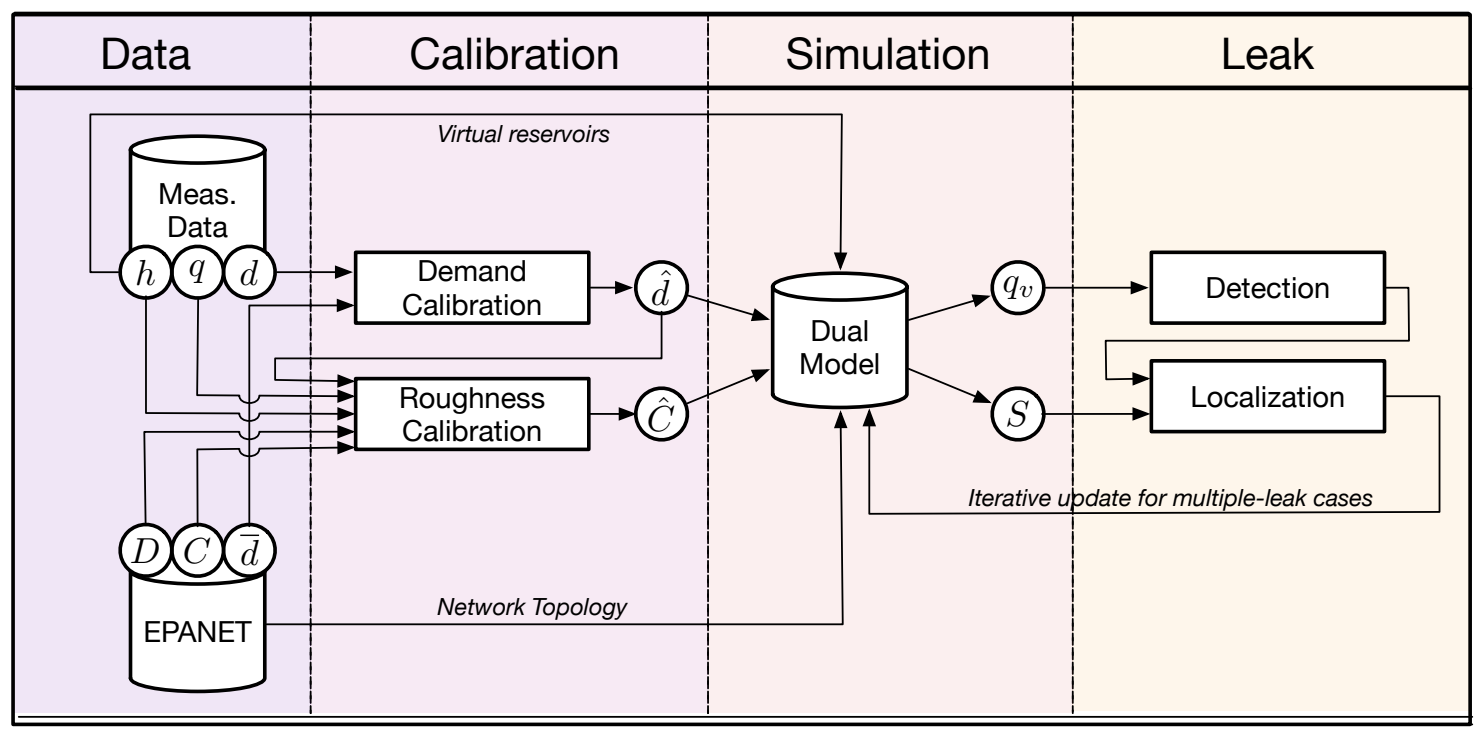

Fig. 1. Overview of the hierarchical leak detection and isolation approach from left to right: Starting with the data analysis (measurements and EPANET model), then model calibration (nodal demand and pipe roughness), followed by simulations with the dual model approach, to finally detect and localize leaks. 

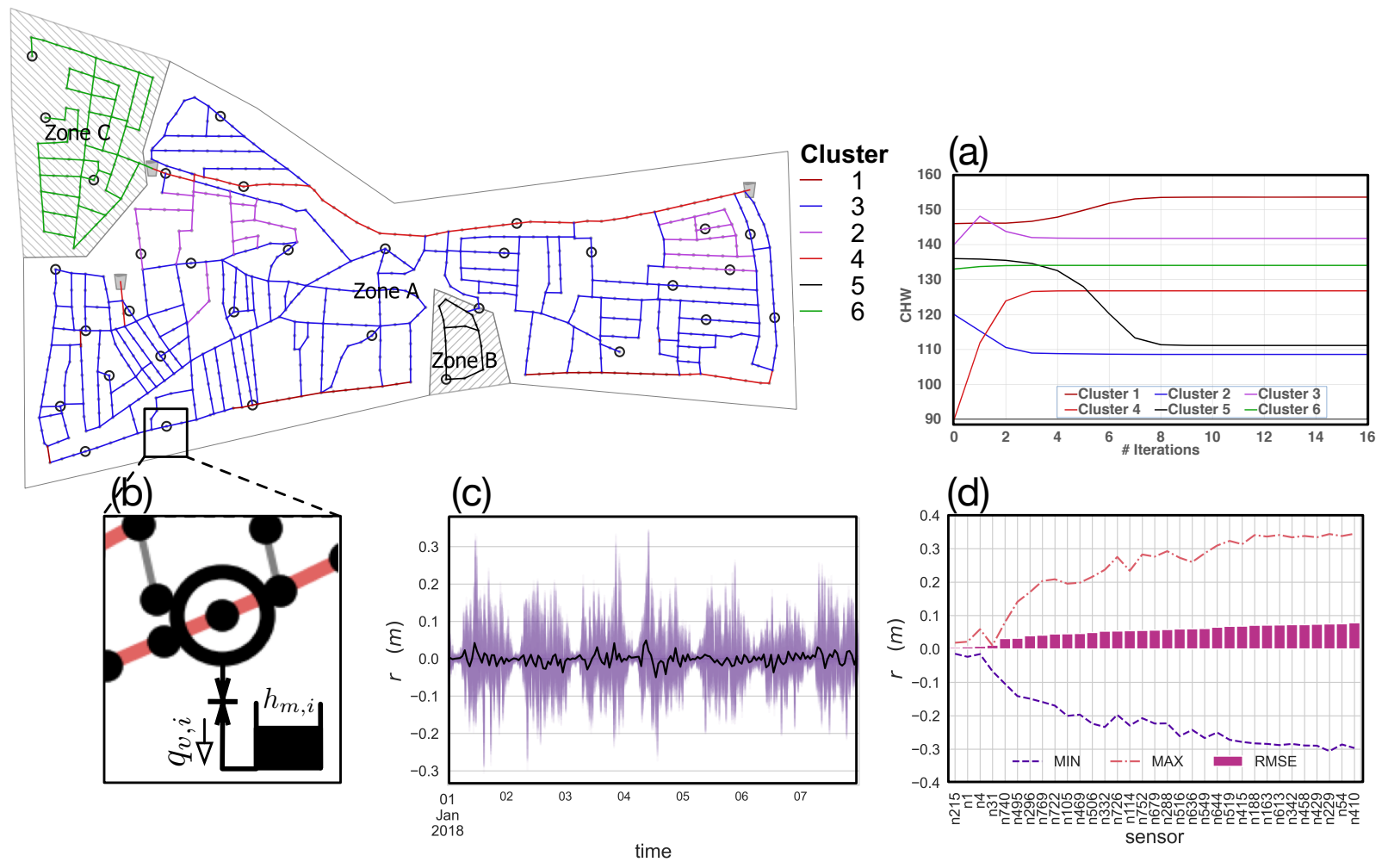

Fig. 2. Network colored by calibration clusters of Hazen-Williams roughness coefficients. Pressure measurements are shown as circles. In a) the roughness iterations are plotted; in b), the inset shows the principle of the dual model, where the pressure measurements are replaced by the combination of a valve and a virtual reservoir whose head is equal to the measured head $h_{i}$; the dual model transforms $h_{i}$ into virtual leakage flows $q_{v_{i}}$; in (c) the pressure residuals are shown for the first week of 2018; and finally, in (d) the minimum, maximum, and root mean square errors (RMSE) are shown in increasing RMSE order. 

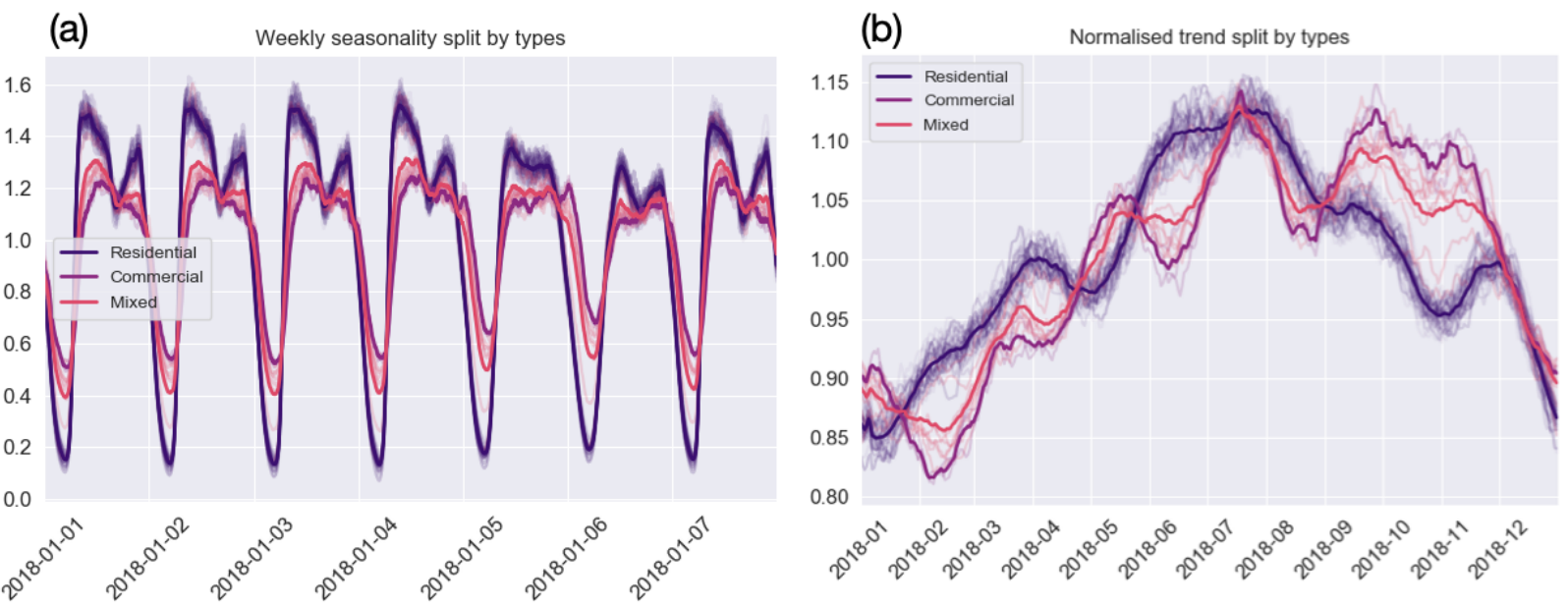

Fig. 3. Weekly seasonality (a) and yearly trend (b) extracted from the AMR measurements for the different customer types (Residential and Commercial) and nodes consisting of a mix of them (Mixed). 

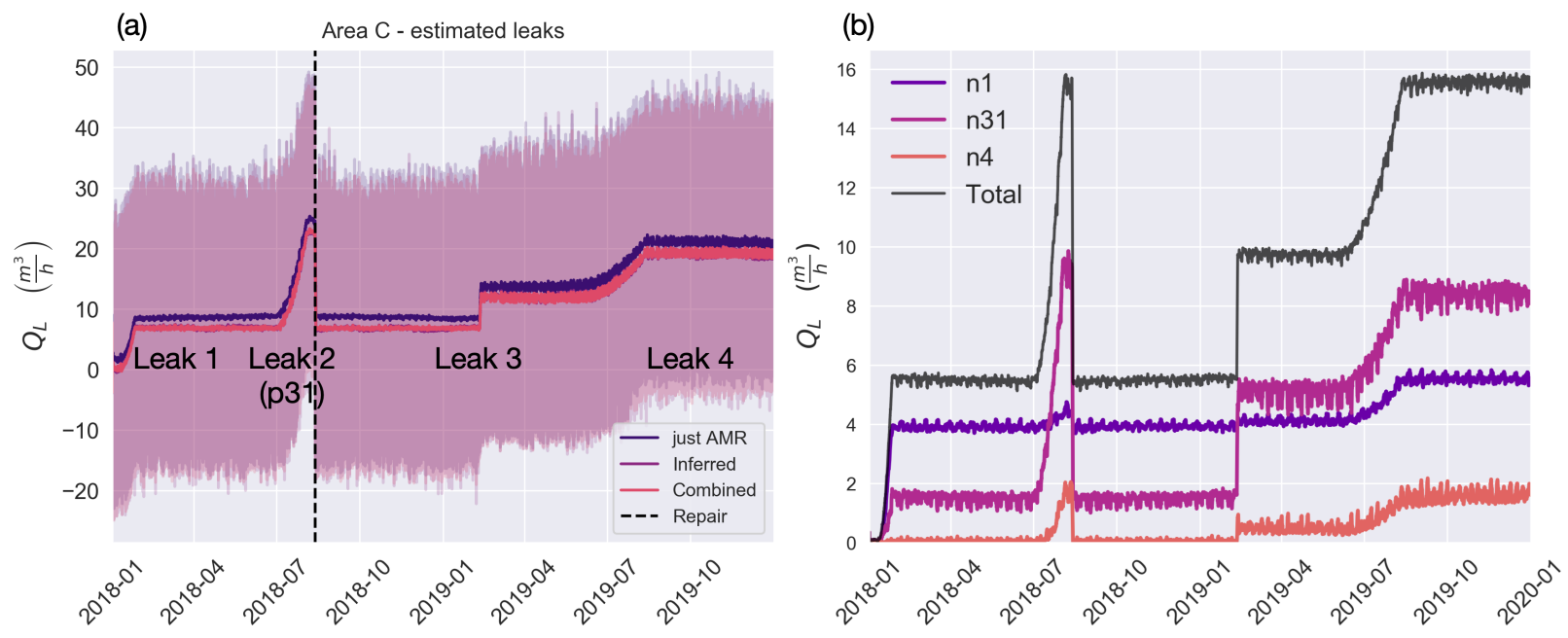

Fig. 4. Leakage outflow in Area C (a) estimated by comparing the "virtual" inflow measurement and the demand model and (b) as provided by the dual model. 

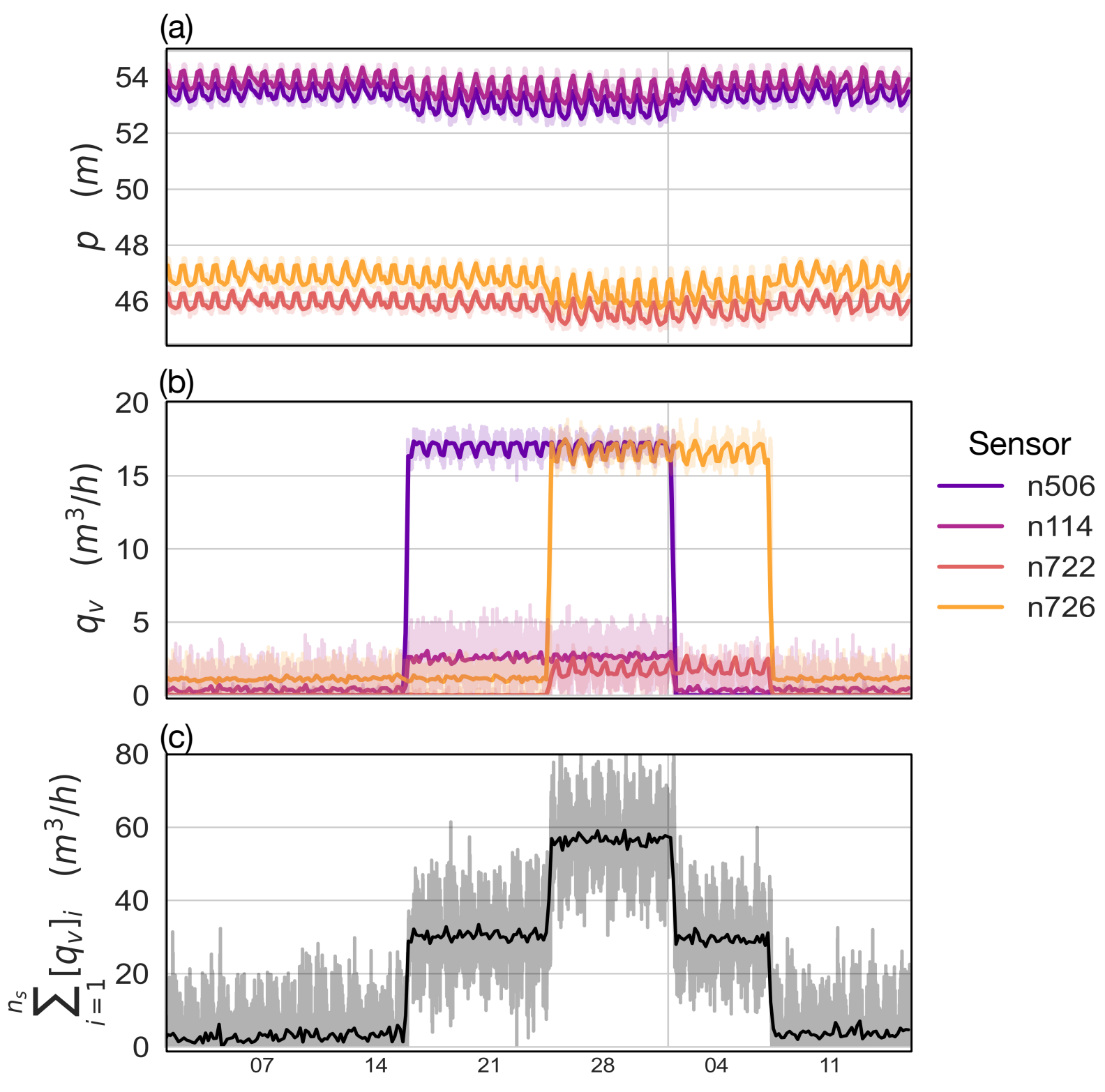

Jan

Feb 2019

\section{time}

Fig. 5. Dual model signals for first two leaks in Area A in 2019 (location at pipes p 827 and p523 with magnitudes of approximately $27 \mathrm{~m}^{3} / h$ each). (a) Pressure measurements $p$ over time, (b) sharp and localized signal of the virtual leak outflows $q_{v}$ over time calculated by the dual model at the same measurement locations, (c) the sum over all virtual leak flows in the dual model serves as good estimates for leak size. 


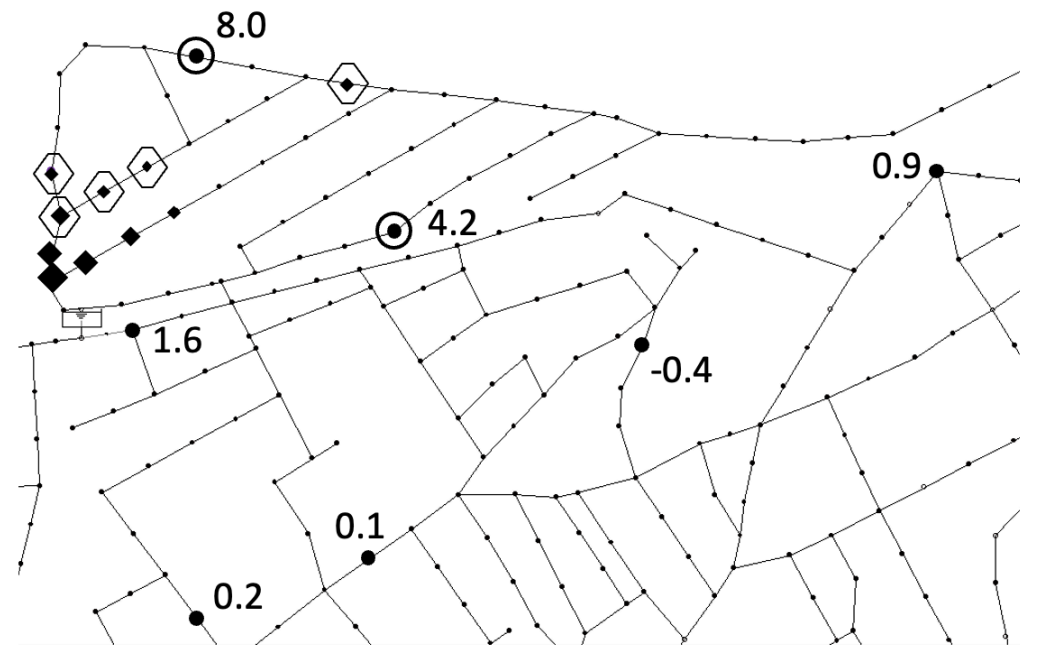
Nodes with highest
- correlations over all
time steps (size $=$ score)
Nodes with highest
- correlations at current time step
Sensor nodes with
0.1 calculated outflows [L/s]
Sensor nodes with
(-) calculated outflow above $5 \mathrm{~L} / \mathrm{s}$

Fig. 6. Snapshot of the leakage isolation tool: calculated outflows at virtual reservoirs of sensor nodes and correlation results: small diamonds for current time step and large diamonds for sum of all time steps (the size represents the score). 
(a)

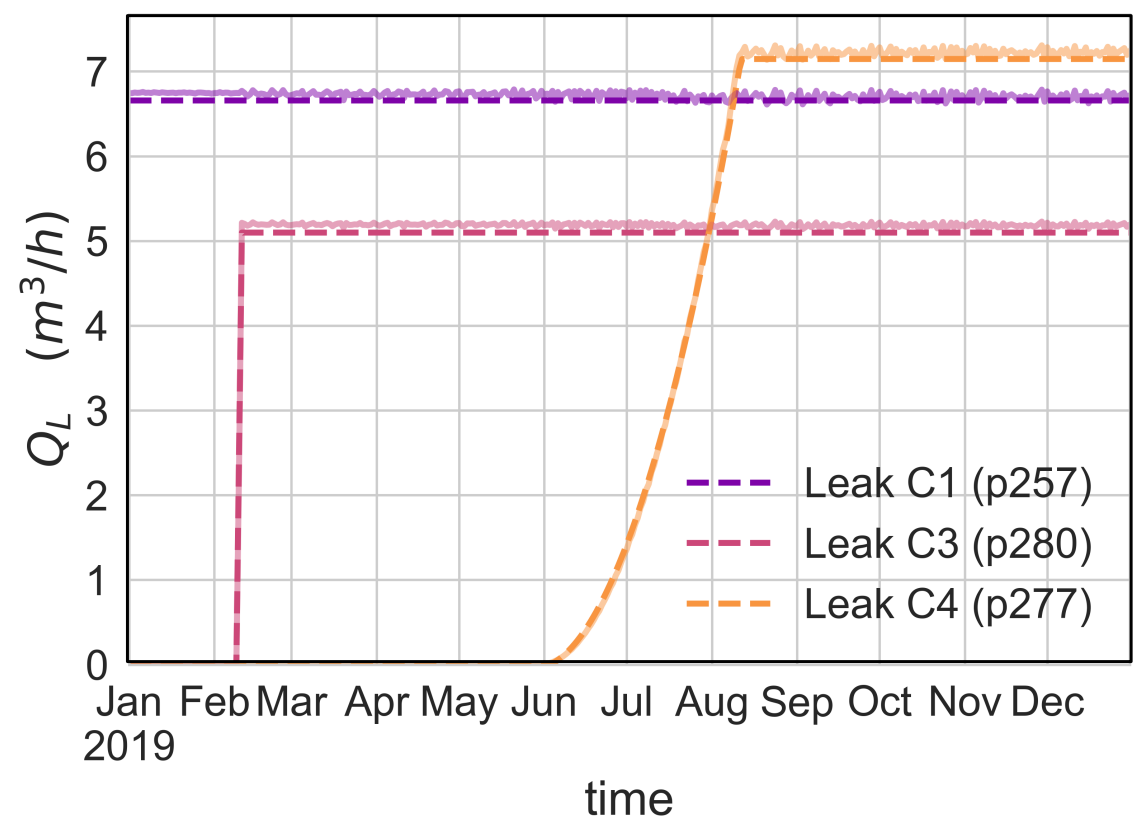

(b)

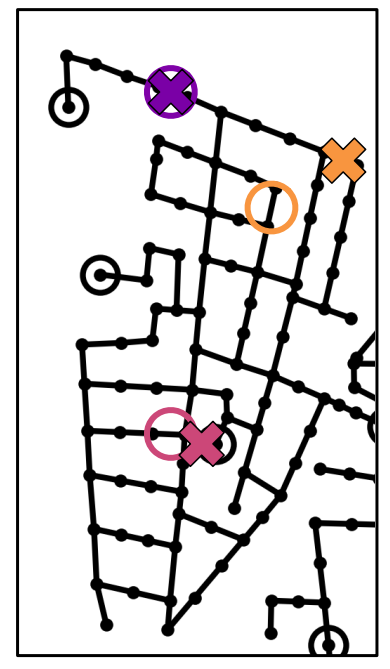

Fig. 7. Results of leak detection and localization for the unknown leaks in Area C in 2019: (a) Identified leakage outflows over time and (b) estimated locations of the leaks. Crosses are the estimated leak locations, circles indicate the real locations. 
(a)

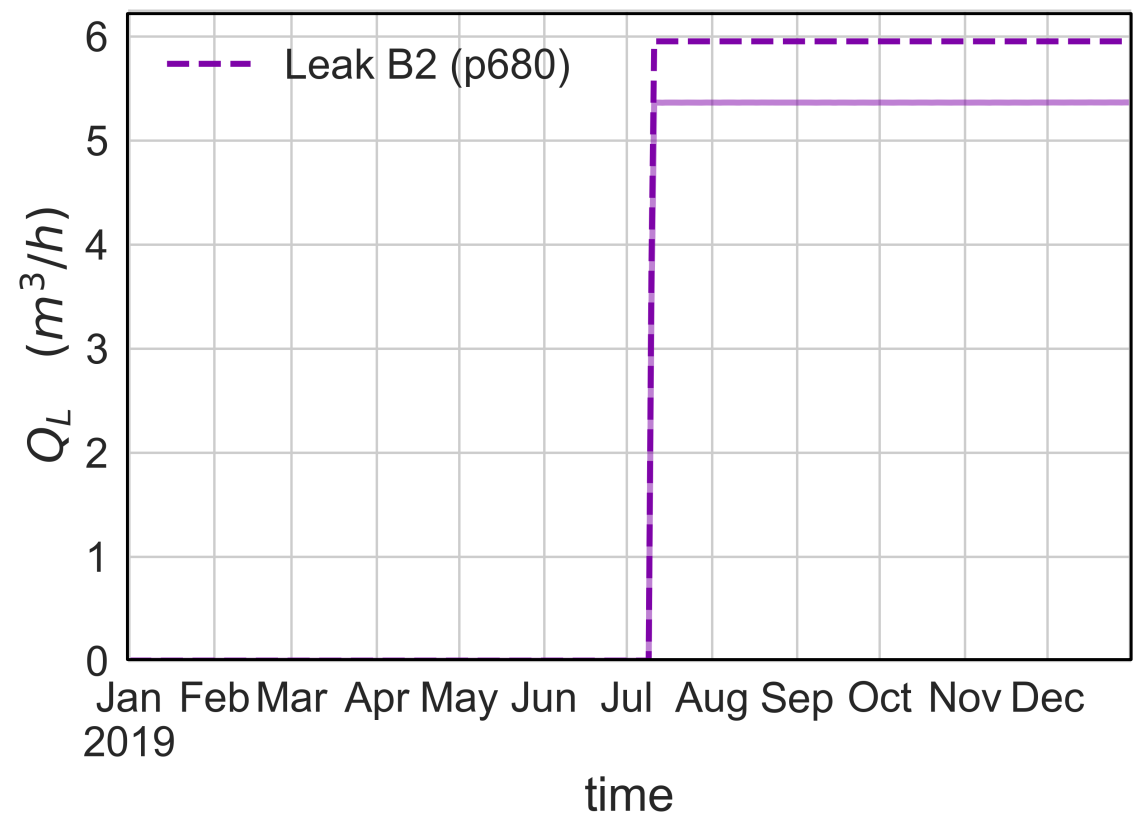

(b)

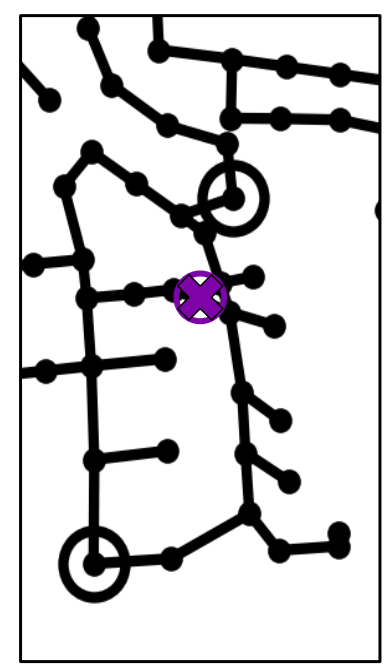

Fig. 8. Results of leak detection and localization for the unknown leaks in Area B in 2019: (a) Identified leakage outflows over time; and (b) estimated locations of the leaks. The Cross is the estimated leak location, the circle indicates the real location. 

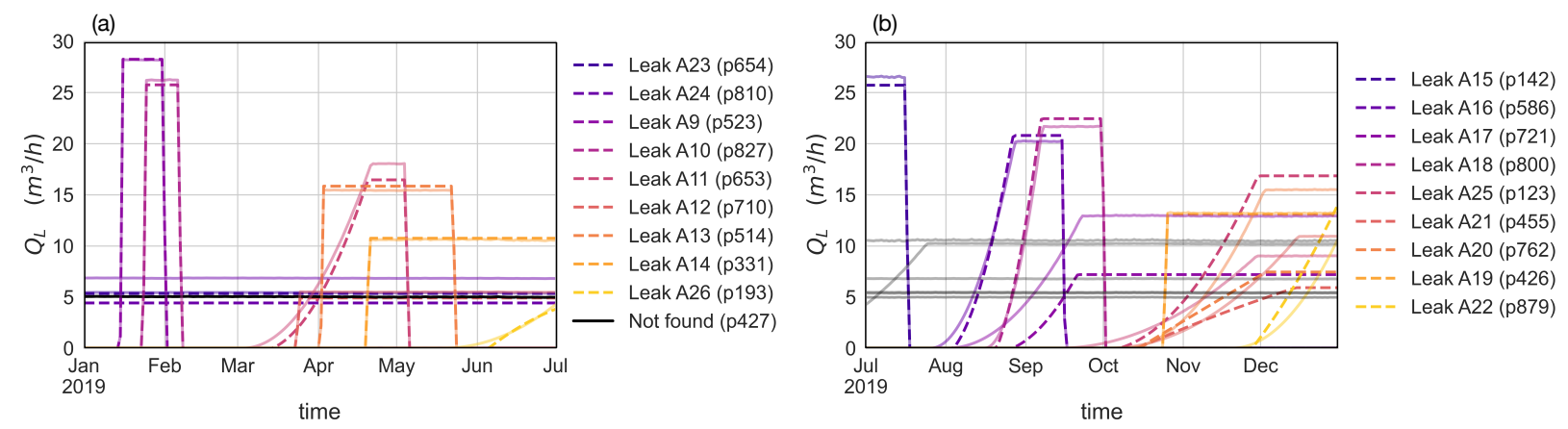

Fig. 9. Results of leak detection for the unknown leaks in Area A in 2019: (a) Leakage outflows for the first half of the year / leaks, and (b) for the second half of the year / leaks. The second half also includes the ongoing leaks from (a) as shaded lines. Additionally, the missed detected leak at pipe $\mathrm{p} 427$ is shown in (a). 
(a)

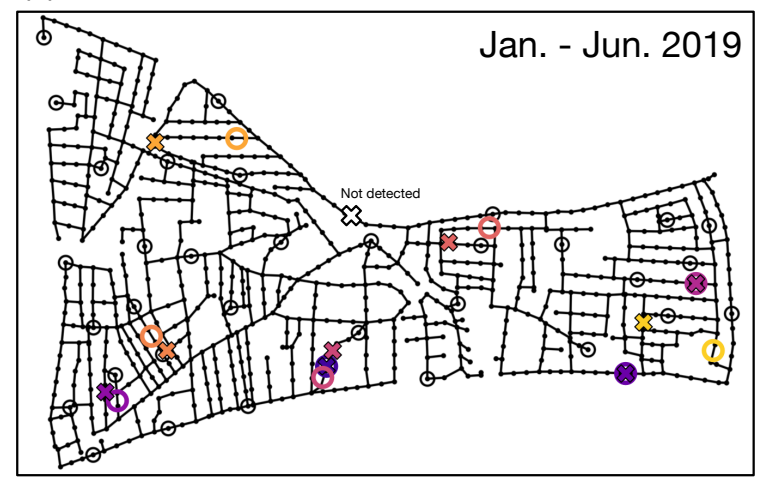

(b)

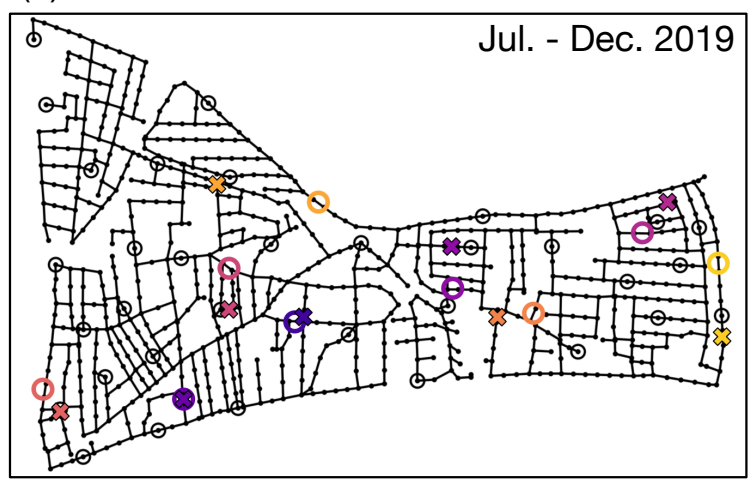

Fig. 10. Results of leak localization for the unknown leaks in Area A in 2019: (a) First half of the year from January to June, and (b) for the second half of the year from July to December. The not detected leak at pipe 427 is shown as a white cross in (a). Crosses are the estimated leak locations, circles indicate the real locations. 


\title{
PRESSURE-LEAK DUALITY FOR LEAK DETECTION AND LOCALIZATION IN WATER DISTRIBUTION SYSTEMS
}

\author{
David B. Steffelbauer ${ }^{1,2}$, Jochen Deuerlein ${ }^{3,5}$, Denis Gilbert ${ }^{4}$, Edo Abraham ${ }^{2}$, and Olivier Piller ${ }^{4,5}$ \\ ${ }^{1}$ Department of Civil and Environmental Engineering, Norwegian Univ. of Science and \\ Technology (NTNU), S.P. Andersens veg 5, 7031 Trondheim, Norway. Email: \\ david.steffelbauer@ntnu.no \\ ${ }^{2}$ Water Management Department, TU Delft, Stevinweg 1, 2628 CN, The Netherlands. \\ 33S Consult GmbH, Albtalstrasse 13, 76137 Karlsruhe, Germany \\ ${ }^{4}$ INRAE, ETBX Research Unit, Aqua Department, F-33612 Cestas, France \\ ${ }^{5}$ School of Civil, Environmental and Mining Engineering, University of Adelaide, South Australia \\ 5005, Australia
}

\begin{abstract}
Water utilities are challenged to reduce their water losses through detecting, localizing, and repairing leaks as fast as possible in their aging distribution systems. In this work, we solve this challenging problem by detecting multiple leaks simultaneously in a water distribution network for the Battle of the Leak Detection and Isolation Methods. The performance of leak detection and localization depends on how well the system roughness and demand are calibrated. In addition, existing leaks affect the diagnosis performance unless they are identified and explicitly represented in the model. To circumvent this "chicken-and-egg" dilemma, we decompose the problem into multiple levels of decision making (a hierarchical approach) where we iteratively improve the water distribution network model and so are able to solve the multi-leak diagnosis problem.

First, a combination of time series and cluster analysis is used on smart meter data to build patterns for demand models. Second, point and interval estimates of pipe roughnesses are retrieved
\end{abstract}


using least squares to calibrate the hydraulic model, utilizing the demand models from the first step. Finally, the calibrated primal model is transformed into a dual model that intrinsically combines sensor data and network hydraulics. This dual model automatically converts small pressure deviations caused by leaks into sharp and localized signals in the form of virtual leak flows. Analytical derivations of sensitivities with respect to these virtual leak flows are calculated and used to estimate the leakage impulse responses at candidate nodes. Subsequently, we use the dual network to (i) detect the start time of the leaks and (ii) compute the Pearson correlation of pressure residuals, which allows further localization of leaks. This novel dual modeling approach resulted in the highest true-positive rates for leak isolation among all participating teams in the competition.

\section{INTRODUCTION}

The detection, localization and control of leakage from aging water distribution networks (WDNs) remains one of the main challenges for water utilities (WUs), because the direct financial cost of water loss can be high. By detecting and dealing with leaks and bursts fast, utilities can also mitigate deterioration of pipes and surrounding infrastructure in addition to lost revenues (Gupta and Kulat 2018). The aim to reduce leakage is further motivated by stringent regulations and financial incentives (OECD 2016).

Conventional techniques for detecting leakage include random and regular sounding surveys using listening sticks and acoustic loggers (Adedeji et al. 2017), and step-testing of metered subsystems as district metered areas (DMAs) through gradual valve closures (Farley and Trow 2003; Wu 2008). More advanced leakage pin-pointing methods like leak noise correlators, pigmounted acoustic sensing and gas-injection techniques (Puust et al. 2010) are the most precise at locating leaks. However, all these techniques come with expensive equipment cost and are manhour intensive, and so are not scalable. In addition, the suppression of leakage sound signatures by reduced pressures in active pressure management or increasing use of plastic pipes in the network has also made these methods less effective (Wu 2008; Puust et al. 2010).

More recent advanced approaches use model-based analysis of near real-time telemetry data 
from pressure sensors and flow meters distributed over the network. Starting with the work of Pudar and Liggett 1992, model-based leak localization was intensively studied with diverse set of methods ranging from sensitivity matrix-based approaches (Pérez et al. 2011; Perez et al. 2014), meta-heuristic optimization (Wu 2008; Steffelbauer and Fuchs-Hanusch 2016b), error-domain model falsification (Goulet et al. 2013; Moser et al. 2017), to combinations of model-based and data-driven approaches (Soldevila et al. 2016; Soldevila et al. 2017). An extensive review of leak localization techniques including their limitations can be found in $\mathrm{Hu}$ et al. 2021. This manuscript deals with a novel model-based approach that leverages time-series analysis of demand models and new hydraulic modeling approaches for both detecting and localizing potential leaks. One of the main challenges for model-based leak detection approaches is the sparse number of pressure sensors compared to the number of candidate leak location nodes. For methods that solve for multiple leaks by posing inverse problems to determine leak parameters in the network model (Pudar and Liggett 1992) (i.e. leak levels and locations), this creates an under-determined and ill-posed problem. Additionally, the performance of model-based approaches can also be very sensitive to errors in two important model parameters: the demand at nodes and pipe roughness coefficients (Hutton et al. 2014). Sanz et al. 2016 reduce this error by including existing leaks in the calibration process. This is done by co-optimizing the calibration and detection, and updating the calibrated model through iteration as new data becomes available and leaks are discovered and fixed. This is achieved through an iterative calibration process, where demands at nodes are composed of geographically distributed demand components. Due to the fact that a leak occurs as a less geographically spread component in this approach, they become easier to find. The method of Sanz et al. 2016 belongs to a class of methods that rely on first-order pressure sensitivities to changes in demand at nodes, and the projection of pressure residuals (differences of measured pressures from leak free case, usually retrieved from time series or well calibrated hydraulic models) onto the sensitivities (Sanz et al. 2016). However, this class of methods have the limitations that they assume a single leak in the system at one time, and are known to be less reliable for small leak sizes, since the leak induced pressure deviations and, hence, the pressure residuals are very small in that case. 
In this manuscript, we address these limitations of pressure residual projection approaches (i.e., the applicability on single as well as small leaks) by combining multiple methods. As in Sanz et al. 2016, we utilize an iterative calibration of the system roughness and demand parameters using multiple measurements, including automatic meter readings (AMRs). To deal with multiple leaks, we separate the detection and localization process; time series analysis (TSA) is used to automatically find deviations in demand and flow measurements, thus, estimating the start and end time of multiple growing and non-growing leaks that can coincide. The detected leaks are then localized by using a residual projection approach (Steffelbauer et al. 2020), where the model is updated when leaks are discovered or fixed. A new duality-based approach is then proposed to improve the sensitivity of the localization process to smaller leaks. We formulate a dual network model, where thanks to a mathematical trick — by transforming the network model with pressure measurements to an equivalent model with additional virtual reservoirs and valves — we are able to translate pressure heads directly to virtual leakage outflows at the measurement locations, which provide a first estimate for the leak's size and location in the network.

Subsequently, we use the virtual leak flows of the dual model for leak detection with anomaly detection algorithms (i.e., the cumulative sum control chart (CUSUM) algorithm and the likelihood ratio test (Peach et al. 1995)) to obtain information on the leak start-time; and the residual-based localization to retrieve the location of the leak. Finally, the information from the detection and localization methods are combined to get accurate estimates for the actual size and location of the leaks.

In the next section, an exposition of the different methods will be presented. We will then discuss the results using the L-Town network model of the Battle of the Leak Detection and Isolation Methods (BattLeDIM) competition (Vrachimis et al. 2020), which the authors of this manuscript won under the team name Under Pressure. The final section will present the conclusions, limitations and future directions to improve the proposed method.

\section{METHODS}




\section{Overview}

We solve the leak detection and isolation problem through utilizing a hierarchical approach. An overview of the two stages where different methods are combined as well as the order in which they are applied is illustrated in Figure 1, depicting how we attempted to find leaks in the measurement data via model calibration and then simulation with the dual model. In the first stage, the hydraulic model is calibrated, since a well-calibrated model is essential to reliably localize leaks (Savic et al. 2009). The model is itself calibrated in two-stages; starting with demand calibration and followed by pipe roughness parameter estimation. The demand calibration method makes use of TSA on AMR data $d$, and infers estimated demands $\hat{d}$ to unmeasured nodes with respect to their average demand $\bar{d}$ stored in the EPANET file. The pipe roughnesses $\hat{C}$ are estimated through solving a differentiable, constrained, weighted least squares (WLS) problem, which uses the estimated demands $\hat{d}$, measured pressure heads $h$, and the initial roughness values $C$ as found in the original EPANET file. In the second, a dual model is built based on the calibrated values $(\hat{d}$ and $\hat{C})$ and used for leak detection and localization, where pressure measurements are replaced with virtual reservoirs. The dual model magnifies leak signals by transforming pressures in virtual leakage outflows $q_{v}$. Moreover, dual model leak sensitivities $S$ are computed. Finally, the sensitivities $S$ and virtual flows $q_{v}$ are used to locate the leaks with a correlation-based method similar to Sanz et al. 2016. In cases with multiple leaks that appear simultaneously, the leaks are localized one by one, eliminated from the dual model, and the remaining leaks are detected and located subsequently through an iterative approach.

\section{Calibration}

\section{Nodal demand calibration}

The AMRs data is used to develop a demand model through TSA for the unmeasured customers within the network. Various time series models (Shumway and Stoffer 2010) are tested on the AMRs aiming to extract weekly seasonalities and yearly trends for different customer types (e.g., residential, commercial). The best performance is achieved with a rather simple model, consisting of a multiplicative superposition of weekly seasonalities $(S(t))$, a time varying trend $(T(t))$ and a 
random component $(R(t))$ accounting for stochastic variations and measurement noise

$$
d(t)=\bar{d} \cdot T(t) \cdot S(t) \cdot R(t)
$$

with $\bar{d}$ being the customer's base demand. For each AMR time series, the trend component $T(t)$ is estimated using a convolution filter and subsequently removed by dividing the original time series through $T(t)$, followed by estimation of $S(t)$ through periodical averages over the trend-free series (Seabold and Perktold 2010). After removing the seasonal component by dividing the trendfree series by $S(t)$, only the random component $R(t)$ remains. Subsequently, similarities in the individual seasonal patterns are identified through time series clustering (Steffelbauer et al. 2021). Furthermore, cluster analysis is used to identify the number of distinct patterns $n_{d}$ and outliers. For each demand node $i$ of the network model, a time-varying demand time series $\hat{d}_{i}$ is built as a superposition of the distinct patterns weighted by their individual averages $\bar{d}_{i j}$ associated with the patterns

$$
\hat{d}_{i}(t)=\sum_{j=1}^{n_{d}} \bar{d}_{i j} \cdot T_{j}(t) \cdot S_{j}(t)
$$

Note that the random time series components are neglected when building the estimates $\hat{d}_{i}$.

\section{Pipe roughness calibration}

Pipes with the same material, age, diameter, hydraulic conditions and locations are grouped in clusters with the same roughness value (in this case a Hazen-Williams (HW) coefficient)

$$
\mathbf{C}_{H W}=\mathbf{M}_{H W} \mathbf{x}
$$

where $\mathbf{M}_{H W}$ is the membership matrix of the $n_{p}$ pipes to $n_{c}$ clusters of $\mathrm{HW}$ coefficients, $\mathbf{x} \in \mathbb{R}^{n_{c}}$ is the vector of roughness cluster values to calibrate, and $\mathbf{C}_{H W} \in \mathbb{R}^{n_{p}}$ is the vector of $\mathrm{HW}$ coefficients of pipes. Roughness calibration aims to fit the measurements by adjusting the roughness coefficients 
of the hydraulic model. Following nonlinear regression equations have to be considered

$$
\left[\mathbf{z}^{j}\right]_{i}=\left[\mathbf{S} \mathbf{y}\left(t_{j}, \mathbf{x}\right)\right]_{i}+\varepsilon_{i j}
$$

where $\mathbf{y}\left(t_{j}, \mathbf{x}\right)$ is the hydraulic state that is implicitly defined by the extended period simulations at time $t_{j}, \mathbf{z}^{\mathbf{j}} \in \mathbb{R}^{n_{m}}$ is the vector of measurements at time $t_{j}, \mathbf{S}$ is the selection matrix to select state vectors that correspond to the measurements, and $\varepsilon_{i j} \sim \mathcal{N}\left(0, \sigma_{i j}{ }^{2}\right)$ are independent and identically distributed Gaussian error terms with zero expectation and standard deviation $\sigma_{i j}$.

The box-constrained WLS problem for parameter calibration consists of seeking to minimize the differentiable criterion

$$
\min _{\mathbf{x}^{L} \leqslant \mathbf{x} \leqslant \mathbf{x}^{U}} \mathrm{f}(\mathbf{x}) \triangleq \frac{1}{2} \sum_{j=1}^{n_{t}} \sum_{i=1}^{n_{m}} \mathrm{H}_{\kappa}\left(\frac{\left[\mathbf{S y}\left(t_{j}, \mathbf{x}\right)\right]_{i}-\left[\mathbf{z}^{j}\right]_{i}}{\sigma_{i j}}\right)+\frac{\alpha}{2}\left\|\mathbf{x}-\mathbf{x}^{0}\right\|_{2}{ }^{2},
$$

where in place of the traditional least-squares criterion the weighted Huber function $\mathrm{H}_{\kappa}$ with parameter $\kappa$ is used, as in Preis et al. (2011), to increase the robustness of parameter estimates against outliers, $n_{t}$ is the number of observation times, $n_{m}$ the number of measurements, $\mathbf{x}^{L}$ and $\mathbf{x}^{U}$ are the lower and upper bounds, $\mathbf{x}^{0}$ is prior information about $\mathbf{x}(e . g$. initial value in the EPANET file) and $\alpha$ is a Tikhonov regularization coefficient, which penalizes large departures from $\mathbf{x}^{0}$ for sufficiently large $\alpha$ and increases the robustness of parameter estimates against outliers. The state of the art algorithm for solving a differentiable WLS problem is the iterative Levenberg-Marquardt algorithm. At each iteration step, the gradient of $\mathrm{f}$ is calculated to estimate the Hessian at the last estimate $\mathbf{x}^{k}$. The gradient of $\mathrm{f}$ at $\mathbf{x}^{k}$ is:

$$
\nabla \mathrm{f}^{k}=\sum_{j=1}^{n_{t}} \mathbf{J}\left(t_{j}, \mathbf{x}^{k}\right)^{T} \mathbf{W}_{j} \tilde{\mathbf{R}}\left(t_{j}, \mathbf{x}^{k}\right)+\alpha\left(\mathbf{x}^{k}-\mathbf{x}^{0}\right)
$$

where $\mathbf{W}_{j}$ is the diagonal weight matrix at time $t_{j}, \mathbf{J}\left(t_{j}, \mathbf{x}^{k}\right)=\mathbf{S} \partial_{x} \mathbf{y}\left(t_{j}, \mathbf{x}^{k}\right)$ is the Jacobian matrix of the prediction function at $\mathbf{x}^{k}$, with $\partial_{x} \mathbf{y}$ using the postmultiplication by $\mathbf{P}=\mathbf{M}_{H W}$ as in Piller et al. 
${ }_{167}$ (2017), and $\tilde{\mathbf{R}}\left(t_{j}, \mathbf{x}^{k}\right)$ is the $\left(n_{m}, 1\right)$-vector of truncated unreduced residuals

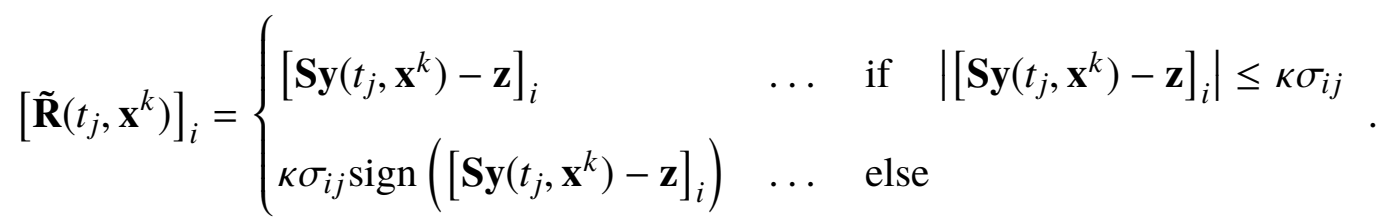

The estimate of the Hessian is following symmetric, positive definite matrix:

$$
\mathbf{H}_{k}=\sum_{j=1}^{n_{t}} \mathbf{J}\left(t_{j}, \mathbf{x}^{k}\right)^{T} \mathbf{W}_{j} \tilde{\mathbf{J}}\left(t_{j}, \mathbf{x}^{k}\right)+\alpha \mathbf{I}_{n c}=\sum_{j=1}^{n_{t}} \tilde{\mathbf{J}}\left(t_{j}, \mathbf{x}^{k}\right)^{T} \mathbf{W}_{j} \tilde{\mathbf{J}}\left(t_{j}, \mathbf{x}^{k}\right)+\alpha \mathbf{I}_{n c},
$$

where $\tilde{\mathbf{J}}$ is given by

$$
\left[\tilde{\mathbf{J}}\left(t_{j}, \mathbf{x}^{k}\right)\right]_{m n}=\left\{\begin{array}{lll}
{\left[\mathbf{J}\left(t_{j}, \mathbf{x}^{k}\right)\right]_{m n}} & \ldots & \text { if }\left|\left[\mathbf{S y}\left(t_{j}, \mathbf{x}^{k}\right)-\mathbf{z}\right]_{m}\right| \leq \kappa \sigma_{m j} \\
0 & \ldots & \text { else }
\end{array}\right.
$$

The constraints are taken into account through a saturation/desaturation process by checking the Karush-Kuhn-Tucker optimality conditions to identify the optimal Lagrange multipliers.

The projected Levenberg-Marquardt algorithm consists of solving following linear system

$$
\mathbf{x}^{k+1}=\mathbf{x}^{k}-\mathbf{C}_{k}^{T}\left(\mathbf{C}_{k} \mathbf{H}_{k} \mathbf{C}_{k}^{T}\right)^{-1} \mathbf{C}_{k} \nabla \mathbf{f}_{k}
$$

where $\mathbf{C}_{k}$ is the selection matrix for the unsaturated components $\mathbf{x}^{k}$. To cope with ill conditioned Hessians, a damping factor with a regularization parameter is introduced to scale the gradient according to the curvature

$$
\mathbf{H}_{k}(\lambda)=\mathbf{H}_{k}+\lambda\left[\operatorname{diag}\left(\mathbf{H}_{k}\right)+\phi \mathbf{I}_{n_{c}}\right]
$$

where $\phi$ is a positive parameter and $\lambda$ is the damping parameter. Furthermore, we make use of 
following relation to calculate confidence intervals for the roughness estimates (Piller 2019)

$$
-\left[\boldsymbol{\Delta}_{x}\right]_{i} \leqslant \delta \mathbf{x}_{i} \leqslant\left[\Delta_{x}\right]_{i}, \text { with } \mathbf{M}=\left(\mathbf{W}^{0.5} \mathbf{J}\right)^{+},\left[\boldsymbol{\Delta}_{x}\right]_{i}=3 \sum_{k=1}^{n_{m}}\left|\mathbf{M}_{i k}\right|, \mathrm{i}=1, \cdots, n_{c}
$$

with $\mathbf{J}$ is the block matrix $\mathbf{J}=\left(\begin{array}{lll}\mathbf{J}\left(t_{1}, \mathbf{x}\right)^{T} & \cdots & \mathbf{J}\left(t_{n_{t}}, \mathbf{x}\right)^{T}\end{array}\right)^{T}$ and $\mathbf{W}^{0.5}$ is the diagonal matrix $\mathbf{W}^{0.5}=\left(\mathbf{W}_{j}^{0.5}\right)=\left(\sigma_{i j}\right)$.

\section{The Dual Model}

We introduce a so-called "Dual Approach (DA)" for detecting and localizing leaks, that is depicted in Figure 2 (b). In the DA, the model is augmented with $n_{s}$ virtual reservoirs that are connected with pressure measurement nodes by valves. The origin of the name "dual" stems from the fact that, instead of using the fixed demand boundary condition at the sensor nodes (i.e. the original or "primal" hydraulic model), the measured pressure heads are used as fixed head boundary conditions at the corresponding virtual reservoirs. Consequently, the heads at the measurement nodes become free variables and imbalances in the system compared to a leak-free model lead to flows to the virtual reservoirs. If there are no leaks, and if we set the minor loss of each virtual reservoir's valve to zero, the two networks are equivalent. In the hydraulic model, we normally set these valves' minor loss to a sufficiently low but non-zero value, and so the primal and dual networks are 'numerically equivalent' but not mathematically equivalent.

If a new leak appears in the primal model, the residuals between measured and calculated pressures change. The pressure drops caused by higher flow velocities towards the leak in the real system are not observed in the model that is still based on the leak free system. In the dual approach, the measured pressure drop is applied to the fixed head reservoirs and, as a consequence, an additional outflow is generated. This outflow can be understood as an outflow residual or virtual leak flow. The advantage of the DA is that the calculated outflows act as amplifiers that deliver significant and localized signals even for small pressure drops. In addition, the outflows at the virtual reservoirs serve a good first estimate for the leak's size and location. 


\section{Dual Model Sensitivities}

We consider the dual WDN with $n_{p}$ pipes, $n_{s}$ virtual links and $n_{j}$ junction nodes at which the heads are unknown. We also denote the vector of unknown flows in the pipes and virtual links by $\mathbf{q} \in \mathbb{R}^{n_{p}+n_{s}}$, the unknown heads and demands at the (free) nodes by $\mathbf{h} \in \mathbb{R}^{n_{j}}$ and $\mathbf{d} \in \mathbb{R}^{n_{j}}$, respectively. The sensitivities of heads and pipe flow rates with respect to nodal outflows are derived among other sensitivities in Piller et al. (2017). The local sensitivities $\nabla_{\mathbf{d}} \mathbf{h}$ and $\nabla_{\mathbf{d}} \mathbf{q}$ can be calculated in demand driven analysis as follows

$$
\begin{aligned}
& \nabla_{\mathbf{d}} \mathbf{h}=-\left(\mathbf{A}^{T} \mathbf{F}^{-\mathbf{1}} \mathbf{A}\right)^{-1} \\
& \nabla_{\mathbf{d}} \mathbf{q}=-\mathbf{F}^{-1} \mathbf{A}\left(\mathbf{A}^{T} \mathbf{F}^{-\mathbf{1}} \mathbf{A}\right)^{-1},
\end{aligned}
$$

where $\mathbf{A}$ is the link-node-incidence matrix of the dual network graph reduced to junction nodes (all links, including pipes and virtual links, are taken), and $\mathbf{F}$ is the diagonal matrix of head loss derivatives with respect to $\mathbf{q}$.

Let $\mathbf{A}_{f} \in \mathbb{R}^{\left(n_{p}+n_{s}\right) \times\left(n_{f}+n_{s}\right)}$ be the link-node-incidence matrix of the dual network graph reduced to fixed-head nodes (the $n_{f}$ initial tanks and reservoirs, and the $n_{s}$ virtual reservoirs), and let $\mathbf{q}_{\text {in }}=\mathbf{A}_{f} \mathbf{q}$ represent the unknown flow rate entering in the system (leaving the fixed-head nodes if positive). Then the sensitivity of the $\mathbf{q}_{\text {in }}$ can be written as using Eq. (13)

$$
\nabla_{\mathbf{d}} \mathbf{q}_{i n}=-\mathbf{A}_{f}^{\mathbf{T}} \mathbf{F}^{-\mathbf{1}} \mathbf{A}\left(\mathbf{A}^{\mathbf{T}} \mathbf{F}^{-\mathbf{1}} \mathbf{A}\right)^{-\mathbf{1}}
$$

The Jacobian in Eq. (14) is the matrix of first order derivatives of the inflows calculated at virtual pressure nodes at measurement locations and real pressure boundary conditions such as reservoirs. The $(i, j)$ element of $\nabla_{\mathbf{d}} \mathbf{q}_{\text {in }}$ represents the first order change rate of the calculated in- or outflow at a fixed-head node $i$ as a consequence of a change in demand at node $j$.

In the dual model the in- and outflows at virtual reservoir are an indicator for a real existing leak or model errors. In a perfect model, where all the parameters are known, the calculated pressures of the dual model would be exactly the same as the measurements from a primal model. In the 
corresponding dual model, the calculated in- and outflows at junctions would be zero and the primal and the dual models would give approximately the same results (i.e. except for small numerical differences due to the minor losses across the virtual reservoir valves).

In presence of an unknown leak, the measured pressure heads and the values calculated by the leak-free primal model diverge. In the dual model, the pressures at the measurement nodes become free and the measurements are set as virtual fixed heads (Figure 2 (b)). The imbalance caused by the unknown leak is then expressed as in- and outflows calculated at pressure measurement nodes. However, as we have shown in the BattLeDIM (Steffelbauer et al. 2020), the sensitivity is much higher in the dual model. Inverting the problem acts as an amplifier of leaks. Another advantage is that the imbalances and the value in question (leaks) have the same unit of flow. The sum of all the imbalances normally gives a good first estimate of the size of the leak. For explanation of the amplifying effect, a deeper investigation of the equation (14) may be useful: from the balance of inflows and outflows, it is possible to deduce each column of $\nabla_{\mathbf{d}} \mathbf{q}_{\text {in }}$ including the fraction of inand outflows as a response to the change in outflow at the corresponding demand node equation

$$
\mathbf{1}_{n_{f}+n_{s}}{ }^{T} \mathbf{q}_{i n}=\mathbf{1}_{n_{j}}{ }^{T} \mathbf{d} \Rightarrow \mathbf{1}_{n_{f}+n_{s}}{ }^{T} \nabla_{\mathbf{d}} \mathbf{q}_{i n}=\mathbf{1}_{n_{j}}{ }^{T} .
$$

The sum of the column vector must be one. Naturally, the response should be an inflow for all fixed-head nodes.

\section{Leak detection and localization}

\section{Leak detection with the dual model}

Whereas in the past, human operators were in charge of small single supply areas, modern WU employees are responsible for multiple DMAs simultaneously (Bakker et al. 2014). That is why automatic anomaly detection algorithms are of particular interest for providing a rapid response to leaks and pipe burst (Romano et al. 2013). However, a correct estimation of the total leakage outflow over their time of existence (from the start $t_{S}$ until the end $t_{E}$ when they are repaired) is of utmost importance to assess water losses (Hamilton and McKenzie 2014). The correct identification of 
$t_{S}$ is also one of the objectives in the BattLeDIM (Vrachimis et al. 2020). We developed a twostage approach to tackle both tasks: (i) using anomaly detection algorithms to detect leaks as fast as possible, and (ii) using regression analysis to retrieve good leak start time $t_{S}$ estimates. For both approaches the virtual leak flows $\left[\mathbf{q}_{v}\right]_{i}=-\left[\mathbf{q}_{i n}\right]_{i+n_{f}}$ (the dual model's outflows to the virtual reservoirs) are used (see Figure 4, for example).

Two algorithms were used to detect leaks in the $\mathbf{q}_{v}$ : (i) the CUSUM algorithm, where a leak is detected when the cumulative sum of positive and negative differences in the signal exceeds a certain threshold $\tau_{1}$, (ii) and the likelihood ratio test (Peach et al. 1995), where a leak is detected if the ratio between the likelihood of the leak versus the leak-free case exceeds a certain threshold $\tau_{2}$. The ideal thresholds for both methods are obtained through calibration on leak free data.

Visual inspection of the virtual leakage outflows $\mathbf{q}_{v}$ of detected leaks revealed two different types of leaks. The first leak type $T_{I}$ is a sudden pipe burst that happen instantaneously at $t_{S}$

$$
q_{L}(t)=\left\{\begin{array}{l}
0 \quad \text { for } \quad t<t_{S} \\
q_{S} \text { for } t \geq t_{S}
\end{array}\right.
$$

where $q_{L}(t)$ is the leakage outflow over time and $q_{S}$ is the saturated (maximum) leak flow (e.g., Leak 3 in Figure 4). Note that leaks are not modeled as pressure dependent demands in contrast to the leaks generated in the BattLeDIM. The second leak type $T_{I I}$ is a slowly growing leak starting at $t_{S}$ and saturating at a certain time $t_{S A}$, modeled as a piecewise function with a quadratic growth rate before the saturation ((e.g., Leak 1, 2 and 4 in Figure 4).)

$$
q_{L}(t)=\left\{\begin{array}{l}
0 \text { for } t<t_{S} \\
a \cdot t^{2}+b \cdot t+c \text { for } t_{S} \leq t \leq t_{S A} \\
q_{S} \text { for } t>t_{S A}
\end{array} .\right.
$$

The coefficients of the quadratic outflow model connect the curves through following relationships $\mathrm{a}=\left(q_{S}-b\left(t_{S A}-t_{S}\right) /\left(t_{S A}{ }^{2}-t_{S}^{2}\right)\right.$ and $c=-a t_{S}^{2}-b t_{S}$. Additionally, it was found that leaks are 
evolving simultaneously in the system, which makes the detection more difficult. If a single leak evolves over time, a Bayesian inference approach based on Hamilton Monte Carlo (Hoffman and Gelman 2014) is used (e.g. in Area C) to identify the parameters $t_{S}, t_{S A}, q_{S}, a, b$, and $c$ plus the confidence intervals of the leak model parameters. In the case of multiple evolving leaks (Area $A \& B)$, differential evolution is used to identify the best combination of leak outflows over time plus the leak parameters of each single leak (Storn and Price 1997). The identified leak outflows were compared against the outcomes of the DA and subsequently used for the leak localization.

\section{Leak localization with the dual model}

The Pearson correlation for flow and pressure residuals and the first-order estimates using sensitivities are calculated for the localization (Perez et al. 2014). It is more convenient for implementation purposes to work with the pressure residuals and sensitivities of the original measurement nodes instead of using the inflow sensitivities in Eq. (14) (e.g. no need for calculating $\mathbf{A}_{f}$ and changing the set of variable pressure nodes). This does not affect the main idea, because the sensitivity of the head is equivalent to the headloss of the virtual valve and, hence, proportional to the flow sensitivity in the linearized system.

The vector of the sensitivities of measured head is determined by

$$
\nabla_{\mathbf{d}} \mathbf{h}_{\mathbf{m}}=-\mathbf{S}\left(\mathbf{A}^{T} \mathbf{F}^{-\mathbf{1}} \mathbf{A}\right)^{-1}
$$

The term $\mathbf{S}$ is the same selection matrix for the measurement nodes as in Eq. (4).

The difference between Eq. (18) and Eq. (14) consists in the multiplication by the derivative of the valve headloss: $\left([\mathbf{S h}]_{i}-h_{n_{f}+i}^{f}=K_{i}\left|\left[\mathbf{q}_{v}\right]_{i}\right|\left[\mathbf{q}_{v}\right]_{i} \Rightarrow \partial_{d_{j}}\left([\mathbf{S h}]_{i}\right)=-2 K_{i}\left|\left[\mathbf{q}_{v}\right]_{i}\right| \partial_{d_{j}}\left(\left[\mathbf{q}_{i n}\right]_{n_{f}+i}\right)\right.$ ). If the sensitivities following Eq. (18) are used, the pressure residuals are used for the calculation of the correlation, whereas the simulated external flows at the virtual reservoirs are considered in the case of Eq. (14).

It proved to be beneficial to calculate the correlations only for measurement nodes where the leak flow (calculated by the dual model) exceeds a certain threshold (e.g. $0.5 \mathrm{~L} / \mathrm{s})$. This adjustment 
eliminates the noise from the pressure measurements and stabilizes the calculated set of candidates for the unknown leak. The Pearson correlation $\rho_{\mathbf{r}, \mathbf{S}_{(\cdot \mathbf{i})}}$ is calculated as

$$
\rho_{\mathbf{r}, \mathbf{S}_{(\cdot \mathbf{i})}}=\frac{\operatorname{cov}\left(\mathbf{r}, \mathbf{S}_{(\cdot, \mathbf{i})}\right)}{\sigma_{\mathbf{r}} \cdot \sigma_{\mathbf{S}_{(, \mathbf{i})}}}
$$

where $\mathbf{r}$ is the vector of residuals, $\mathbf{S}_{(\cdot, \mathbf{i})}$ is the sensitivity vector of node $i, \operatorname{cov}($.$) is the co-variance$ and $\sigma_{\mathbf{r}}$ and $\sigma_{\mathbf{S}_{(\cdot, i)}}$ are the standard deviations of the residual vector and the sensitivity vector, respectively. The residuals and the sensitivity coefficients are very small. However, this did not show any negative impact in the allocation in our tests. In contrast, the system is stabilized by the additional pressure boundary conditions, which makes the correlation more stable compared to the conventional primal model approach. One important limitation of the correlation method is that it does not work for two or more leaks appearing at the same time. Therefore, a single leak must first be isolated in time from other leaks in order to be localized. The leakage curves that have been calculated for detection serve as a basis for choosing the best time for allocation, and we use a step by step procedure for localizing simultaneously growing leaks.

1. Identification of time interval that starts briefly before the new unknown leak starts and ends before the next leak starts. The time intervals from $t_{S}$ to $t_{S A}$ are found by a combination of CUSUM or likelihood ratio tests with Hamilton Monte Carlo or differential evolution (depending on the single or multiple leak case) as described in the leak detection paragraph in the methods section.

2. Initialize calculation for the selected time interval (load all measurements as well as the estimated demands)

3. Run Extended Period Simulations for selected time interval; for each time step do:

(a) Update boundary conditions via toolkit functions including demand patterns, heads at virtual reservoirs, pump flow.

(b) Update all known leaks with their calculated leak flows as fixed demands and define the 
start time of the unknown leak based on the results of the detection.

(c) Simulation of the time step (here the EPANET toolkit is used) and after each time step with active new unknown leak, calculate correlation in Eq. (19) for all nodes based on the sensitivities.

(d) Consider only the nodes with a correlation score higher than a given minimum threshold (e.g. 0.95) and add those eligible correlations to the sum of correlation taken over all calculated time steps.

4. The node with the highest correlation sum is identified as the candidate for the new leak within this time interval.

5. The new leak is added to the list of known leaks and the leakage flow is considered as known demand for the localization of the next leak and the procedure is repeated from point 1 until all leaks have been identified in the given period.

\section{L-Town case study and measurement data}

The case study network L-Town was provided by the organizers of the BattLeDIM (Vrachimis et al. 2020). L-Town is a small hypothetical town based on a real WDN in Cyprus with approximately 10,000 inhabitants, which receive water from two reservoirs. The WDN consists of pipes with diameters ranging from $63 \mathrm{~mm}$ to $225 \mathrm{~mm}$ and a total pipe length of $43 \mathrm{~km}$. L-Town consists of three distinct hydraulic areas: (i) Area A is the main part of the network, (ii) Area B is a low lying part that is supplied through a pressure reduction valve, and (iii) Area $\mathrm{C}$ is an area with higher elevation that is supplied by an elevated tank fed from Area A through a pumping station. An overview of the network and the location of the three measurement zones can be found in Figure 2.

To enhance the water loss monitoring capabilities, the WU of L-Town installed three flow meters (two at the reservoirs and one at the pumping station), a tank level sensor and 33 pressure sensors (depicted as circles in Figure 2). All sensors measure and transmit data every 5 minutes to the utility's supervisory control and data acquisition (SCADA) system. Additionally, the WU installed 82 smart water meters or AMRs in Area C, measuring three different customer types: residential, 
commercial and industrial. There is no flow meter installed at the tank that feeds Area C. Therefore, a virtual inflow measurement to Area $\mathrm{C}$ has to be reconstructed from the tank level measurements and the inflow measurement measured at the pump that supplies the tank.

The dataset of the BattLeDIM contains two years of sensor data for years 2018 (historical dataset) and 2019 (validation dataset), an EPANET model of the water distribution network, plus the time and repair location of ten pipe bursts that have been fixed in 2018. Three types of leaks exist: (i) small background leaks with $1 \%-5 \%$ of the average inflow, (ii) medium pipe breaks with $5 \%-10 \%$, and (iii) large pipe bursts with leakage flows of more than $10 \%$ of the average system inflow $\left(\approx 180 \mathrm{~m}^{3} / \mathrm{h}\right)$. Large leakages with outflows over $15 \mathrm{~m}^{3} / \mathrm{h}$ are fixed by the water utility after a reasonable amount of time within two months. The leakages have two different time profiles, (i) either abrupt pipe bursts with constant leak flow rates, (ii) or background leakages with growing leak rates which evolve over time until large outflow rates at which they remain constant. In total, 14 leakages occurred in 2018 with outflow rates between 5 to $35 \mathrm{~m}^{3} / \mathrm{h}$, of which 10 leaks have been repaired. The remaining 4 leaks are not repaired and continue into the 2019 validation dataset. The BattLeDIM challenge is to find the 19 leaks that happened in 2019 plus the 4 remaining leaks. The outflows and locations of the 33 leaks can be found in Figures 7 to 10 (dashed lines in the outflow time series plots and circles in the location overview plots). More details on the dataset can be found in (Vrachimis et al. 2020).

\section{RESULTS AND DISCUSSION}

\section{Demand calibration}

Each AMR time series is decomposed into its trend, seasonal (with a period length of a week), and random components using the multiplicative time series model described in Eq. (1). Subsequently, cluster analysis is used to identify similarities in the trend and seasonal patterns. Two distinct demand patterns emerge in the trend $T(t)$ and in the seasonal components $S(t)$, a residential $\left(T_{\mathrm{R}}(t), S_{\mathrm{R}}(t)\right)$ and a commercial $\left(T_{\mathrm{C}}(t), S_{\mathrm{C}}(t)\right)$ one. The seasonal and the trend components are shown in Figure 3 for each AMR measurement. Furthermore, some patterns are found to be a superposition of both pattern types. These patterns belong to houses with mixed user groups (e.g. 
commercial space in the ground floor and apartments in the floors above). Subsequently, these patterns are called mixed patterns. Generally, all demand patterns can be described through the superposition (see Eq.(2)) of the residential and the commercial pattern. During workdays (Monday to Friday), water consumption follows a similar behavior, whereas during the weekend (Saturday and Sunday) higher consumption during late hours occur as the result of night life (see Figure 3 (a)). Furthermore, cluster analysis revealed four outlier pattern in the AMR measurements. After closer examination, these outlier patterns were explained as industrial users with a periodicity differing from a week (i.e. 9, 11 or 13 days). Hence, those industrial users do not follow the same pattern of consumption as described in Eq. (2) and are not further used in the demand modeling. The trend components in Figure 3 (b) show higher water usage during July/August, and lower in December/January.

The demand model is used to model the unmeasured customers within the L-town network. Additionally, a virtual inflow measurement of Area $\mathrm{C}$ has been constructed from the pump flow measurements and the tank's water level. This virtual inflow is used to (i) validate the demand model and to (ii) estimate the leak outflow in Area C. Figure 4 (a) shows the estimated leakage outflow, which is constructed as the difference between the virtual inflow measurement and the total estimated demand for Area C. Three different strategies for the demand estimation are used in Area C. First, only the measured demand at the AMRs is subtracted (just AMR in Figure 4 (a)), which leads to an overestimation or an offset of the leak flow, because of the unmeasured customers. Second, the demand for the whole zone is estimated based on the model as described in Eq. 2 using the base demands from the BattLeDIM EPANET model (Inferred), which leads to a high noise in the leak outflow estimates. Third, the AMR measurements are combined with demand estimates for the unmeasured customers (Combined). The last approach leads to the best leak outflow estimates with low levels of noise as well as no offset. Clearly, four different leaks can be seen in the data, three are growing over time until they are saturated (Leak 1, 2, and 4), and a sudden pipe burst (Leak 3). This information proved to be useful for the leakage modeling (see Eq. (16) and (17)). 


\section{Roughness calibration}

The internal diameters of pipes are nominal diameters defined by a discrete number of values that depend on the manufacturer and the material. In the L-Town INP file, it is assumed that the outside diameters of plastic pipes are entered instead of the inside diameters, which is first corrected with the most usual inside diameter for PVC and PE pipes (see Table 1).

After inspection and several tests, the pipes are divided in six different roughness clusters according to their diameter, material, initial roughness values and managing zones in which they are located : Because of the small number of observations and pipes, one cluster with $C_{H W}=x_{5}$ is assigned for Zone $\mathrm{B}$ and one to Zone $\mathrm{C}\left(x_{6}\right)$. Cluster with same $x_{1}$ roughness value consists of the plastic pipes in Zone A; pipes in cluster 2 are in Zone A with diameters $100 \mathrm{~mm}$ or $150 \mathrm{~mm}$, and original INP roughness $x_{2}=120$. Similarly, pipes in zone A with diameters $100 \mathrm{~mm}$ or $150 \mathrm{~mm}$ and original $C_{H W}=140$ define the cluster 3: $x_{3}=140$. Finally, cluster 4 is made of pipes with internal diameter $200 \mathrm{~mm}$ in Zone A. Figure 2 shows an overview of the roughness groups. Through visual inspection of the measurements from the first week of 2018, it is assumed that no leaks are present in the dataset during that time. Consequently, measurements for this week are used for the roughness calibration. The roughness calibration is performed for the six clusters, $n_{c}=6$, and by solving the WLS problem in Eq. (5) with $\kappa=3, \alpha=0$ and box constraints $x^{L}=60$ and $x^{U}=160$ with the Levenberg-Marquardt method (10). The $n_{s}=33$ pressure measurements in Figure 2 are used $\left(n_{m}=33\right)$. They repeat every five minutes for 7 days $\left(n_{t}=2016\right)$. All measurements are chosen to be of the same accuracy $\sigma_{i j}=1$.

The algorithm converges after 11 iterations. The results are given in terms of estimates in Table 2. For the first cluster, plastic pipes in Zone A, the initial estimate $x_{1}^{0}=146$ belongs to the $99 \%$ confidence interval $[141.9,163.7]$. Consequently, the final estimate 152.8 is not significantly different from the initial value. However, the initial estimates for the other five clusters differ significantly from the point estimates at iteration $k=11$ (they do not belong to the five $99 \%$ confidence intervals). Based on the confidence intervals and the initial estimates, the bold values are selected. The pressure residuals are represented in Figure 2. It can be seen that the mean 
squared error (MSE) is about $6 \mathrm{~cm} \mathrm{H}_{2} \mathrm{O}$.

\section{Virtual leak flows with the dual model}

A dual model is constructed from the EPANET model containing the calibrated pipe roughnesses and demand patterns from the demand calibration. The heads of the virtual reservoirs are set to the corresponding pressure measurements. If leaks appear in the network, the dual model reacts with virtual leak outflows caused by the pressure differences of the hydraulic model and the lower reservoir heads. The virtual leak flows for each sensor location within Area $\mathrm{C}$ are depicted in Figure 4 (b). Furthermore, the total sum of all virtual leak flows is shown. This sum gives a good first approximation of the leak size. The second leak in Area $\mathrm{C}$ was repaired and, hence, its end time and its location (pipe p31) are known. The leak is closest to sensor node n31, which shows the strongest reaction to the leak by producing the biggest virtual outflow. Same reasoning leads to the conclusion that Leak 1 is close to sensor $n 1$, Leak 3 is in proximity of $n 31$, and Leak 4 is somewhere in the middle of all three sensors.

Comparison of Figure 4 (a) with the total virtual leak outflow in (b) shows that the real leakage outflows have similar magnitudes as the virtual outflows. However, the dual model seems to underestimate the real outflows in Area C slightly.

Figure 5 shows an comparison of the effect of leakages on the measured pressure signals versus the virtual leak flows in the dual model for the first two leakages in 2019 that appear in Area A (pipe p523 and p810). In this Figure, solid lines are four hour moving averages, whereas the shaded lines are the original five minutes signal. The dual model amplifies the leak signal compared to the pressures (compare Figure 5 (a) and (b)). Furthermore, the leaks have a more local effect on the virtual leak flows than in the pressures, which allows already a rough estimation of the leak's location. The sum of all virtual leak outflows in Figure 5 (c) gives already a good estimate of the leak sizes, which are approximately $27 \mathrm{~m}^{3} / \mathrm{h}$ for each leak.

\section{Leak Detection}

Two different signals are used for leak detection; (i) the flow residual between the measured inflow and total demand plus already known leaks in an area, (ii) the dual model's outflows to the 
virtual reservoirs (see Figure 4 or Figure 5). Two different types of leaks are found in the data instant bursts and leaks that are growing over time. Growing leakage flows are modeled with the quadratic function in Eq. (17). Data from the dual model is used to identify the leak start times and their shapes (i.e. instant or growing). For that reason, thresholds are extracted from the DA flows at each sensor using the leak free case in the first week of 2018. If the DA signal exceeds the threshold, a leak is detected in the system. The detection time is used as the start time of the leak for our BattLeDIM solution. To estimate the leakage outflow, the start times and the shapes of the leaks are used to fit the leak shape on the flow residuals. If a single leak evolves over time, Bayesian inference is used, for multiple simultaneously appearing leaks, a faster differential evolution is used to identify the best combination of leak outflows over time. The detected leaks are double checked against the DA and subsequently used for the leak localization.

The results for leak detection and localization for 2019 are summarized in Table 3. Additionally, the leak detection and localization results are broken down by the different areas are shown in Figures 7 to 10, where shaded lines are daily moving averages of the real leakages, solid dashed lines are the estimated leakages. Circles in the network maps are the real leak locations, while crosses show our estimates. The leak detection results for Area $\mathrm{C}$ are shown in Figure 7 (a). The shapes of the leaks are resembled very well by our method for all three leaks, and the differences in the final leak outflows are negligible for Area C. The sudden pipe burst (Leak C3 at pipe p280) is detected instantaneously, while the detection of the growing leaks takes a bit longer. Nevertheless, leakages are detected on average within less than 9 days. A less conservative detection threshold potentially decreases the detection time.

The leak detection results for Area B are shown in Figure 8 (a), where the instant pipe burst is perfectly detected, although the leakage outflow is slightly overestimated.

The leak detection results for Area A are shown in Figure 9. For a better visibility of the simultaneously appearing leaks, the Figure is split into the two half-years of 2019, with (a) for the first half until July, and (b) showing the second half of the year. Additionally, the leaks from the first half are depicted as gray shaded lines in Figure 9 (b) as they are still present in the network. Sudden 
pipe bursts are again detected instantaneously, while the thresholds for growing leaks seemed a bit too conservative. However, the shapes of all leaks are very well described through the coefficients that our model found. One leak that started in February 2018 at pipe p427 with a magnitude of $5 m^{3} / h$ is not detected at all. All leak shapes are identified correctly until August, when Leak A17 at pipe p721 appears (see Figure 9 (b)). However, this leak is detected very late and its size is underestimated by almost $5 \mathrm{~m}^{3} / \mathrm{h}$. This influences the detection of subsequent leaks, which results in a decrease in the detection as well as the localization performance. Nevertheless, leakages in Zone A were detected within 10 days on average.

\section{Leak Localization}

For the localization of the leaks the network is divided into two separate parts $(A+B$ and $C)$ and the pump is replaced by the flow measurement for Zone A and B. All calculations are executed by use of EPANET 2.00.12 (Rossman 2000) and the EPANET toolkit integrated in an application for data management and visualization that is exclusively developed for the performance of the project.

Figure 6 visualizes the GUI-output at a certain time step. The circles indicate the locations of the pressure measurement nodes and the numbers show the calculated in- and outflows calculated by the dual model. The two biggest virtual reservoirs outflows are marked by a bigger circle as expected in the neighborhood of these two nodes. The diamonds show the nodes with highest correlation scores at the current time and the bigger diamonds show the nodes with highest correlation sum. Their size is scaled by the sum value which means that they are growing over time.

Figure 7 (b) shows the localization results for Area C. Leak $\mathrm{C} 1$ is perfectly isolated at the real location (p257). Leak C3 is found within $50 \mathrm{~m}$ of the real leak. However, if the closed valve in Area $\mathrm{C}$ is added to the hydraulic model, the isolation of this leak might improve further. Leak $\mathrm{C} 4$ is not localized correctly, since the distance exceeds $300 \mathrm{~m}$ as stated in the BattLeDIM rules. Reasons for that might be that the closed valve is not taken into account, or the fact that we are using demand driven models, while the BattLeDIM organizers used a pressure-driven model. The more leakages occur in the network, the greater the difference between a demand-driven and a pressure-driven demand model become, and the more inexact our localization gets, since the localization errors 
accumulate. On average, all leaks are found within $130 \mathrm{~m}$ of the real leak in Area C. For Area B, the leak is perfectly isolated in time as well as in space (see Figure 8).

The results for Area A can be found in Figure 10, and are split again into half-years. Figure 10 (a) also contains the leak that was not detected by our method (white cross). Early leaks are isolated almost perfectly, while the localization gets worse during later simulations. This might be a consequence of the demand-driven model that is used. For the leaks in Figure 10 (a), the average distance of the real leaks to the estimated leak position is around $150 \mathrm{~m}$. During later simulations, this distance increases to $250 \mathrm{~m}$ (see Figure 10 (b) and Table 3). It has to be noted that a typo occurred while submitting the results for the BattLeDIM. Leak p654 was inserted as p645. Taken this into account, the final score of the Team Under Pressure would even further increase from already the highest rate of true positives of $65 \%$ of all participating teams.

\section{CONCLUSION}

In this work, we present a novel solution to detect and isolate multiple-leaks in WDN that we developed while participating in the BattLeDIM competition. Our method consists of calibrating the nodal demand and pipe roughness, and introducing a dual model for the calibrated primal problem to detect and locate leaks.

The calibration uses time series analysis and cluster analysis to build a multiplicative predictive model for ultimately two network-wide demand models, a residential and a commercial model. This is used for both, (i) modeling unknown demands over time in the hydraulic model, as well as distinguishing leakages and consumption in the measurements. Subsequently, six roughness clusters were calibrated using 33 pressure loggers for the first week of 2018. Confidence intervals are given for the least-squares estimates. The pressure residuals are very well reproduced for the entire week with a small root mean square error of $6 \mathrm{~cm}$.

The core of our method is a dual model that transforms a pressure measurement node into a free junction node plus a link to a virtual reservoir, whose head is equal to the measured values. Significant inflows or outflows, either sudden or gradual, to these virtual reservoirs are indications of leaks. In the dual model, the pressure signal is transformed into a virtual leakage outflow 
signal that is easier to analyze since it amplifies and localizes the effects of leaks in the network. Sensitivities of nodal pressures to virtual outflows are also derived. They are essential to understand the behavior of the model at first order.

For leak detection, the CUSUM algorithm and likelihood-ratio tests are used jointly on the virtual leak flows, where the parameters are tuned to limit the global false positive rate under normal operation conditions. When multiple leaks are present, differential evolution is used to identify the best combination of leak modeling parameters over time (i.e. start times and shapes of leaks over time). These detection methods were employed for both, the primal and the dual data. The localization is achieved by analyzing the correlation between the calibrated pressure (or virtual inlet-outlet model predictions) and the corresponding first-order leakage impulse response predictions at the candidate nodes. This solution recovered $65 \%$ of true leaks with only four false positives in all of 2019, which is a notable result (shared \#1 ranking).

Using a pressure-driven model instead of a demand-driven one, improving the calibration by reliably detecting closed valves, as well as using less conservative threshold parameters for the detection of the growing leaks might increase the already notable result further. Certainly, a lot of potential lies in a deeper understanding of the dual model to further improve the detection and isolation of multiple simultaneously occurring leaks.

With 33 pressure sensors, the BattLeDIM dataset contains an unrealistic high number of sensors in a WDN of that size. Indeed, the leak detection and localization performance will decrease with a lower number of sensors. However, optimal sensor placement algorithms might recover similar leak detection and localization performances with fewer sensors. Furthermore, the BattLeDIM organizers constructed the nodal demand patterns through a superposition of residential and commercial demands multiplied with noise. That is why we were able to almost precisely reconstruct the demands on the unmeasured locations through the information contained in the AMR data with our demand calibration approach. In reality, demand patterns are more variable (Steffelbauer et al. 2021). Consequently, the dual model might perform worse in systems with limited demand information and, hence, less accurate demand models. 
That is why for future work, we want to focus on optimal sensor placement (Steffelbauer and Fuchs-Hanusch 2016a) with the dual model and on applying the dual model on challenging real data sets, with model errors, outliers, uncertainty, and more variable and realistic water demands. Furthermore, we are planning to investigate the importance of each step for the final classification, enhancing the method to reduce the false positive rate, and study the effect of the dual model on fitness landscapes of WDN optimization problems (Steffelbauer and Fuchs-Hanusch 2016b).

\section{DATA AVAILABILITY STATEMENT}

All data, models, or code that support the findings of this study are available from the corresponding author upon reasonable request.

\section{ACKNOWLEDGMENTS}

This project has received funding from the European Union's Horizon 2020 research and innovation programme under the Marie Skłodowska-Curie grant agreement No 707404. The opinions expressed in this document reflect only the author's view. The European Commission is not responsible for any use that may be made of the information it contains. One author of this paper was supported in part by the German Ministry for Education and Research (BMBF Project W-Net 4.0 02WIK1477C). 


\section{APPENDIX}

AMR automatic meter reading

BattLeDIM Battle of the Leak Detection and Isolation Methods

CUSUM cumulative sum control chart

DA Dual Approach

DMA district metered area

HW Hazen-Williams

MSE mean squared error

SCADA supervisory control and data acquisition

TSA time series analysis

WLS weighted least squares

WDN water distribution network

WU water utility

\section{REFERENCES}

Adedeji, K. B., Hamam, Y., Abe, B. T., and Abu-Mahfouz, A. M. (2017). "Towards Achieving a Reliable Leakage Detection and Localization Algorithm for Application in Water Piping Networks: An Overview." IEEE Access, 5, 20272-20285.

Bakker, M., Vreeburg, J. H. G., Van De Roer, M., and Rietveld, L. C. (2014). "Heuristic burst detection method using flow and pressure measurements." Journal of Hydroinformatics, 16(5), 1194-1209.

Farley, M. and Trow, S. (2003). Losses in water distribution networks: A practitioner's guide to assessment. IWA publishing.

Goulet, J.-A., Coutu, S., and Smith, I. F. (2013). "Model falsification diagnosis and sensor placement for leak detection in pressurized pipe networks." Advanced Engineering Informatics, 27(2), 261269. 
Gupta, A. and Kulat, K. D. (2018). "A selective literature review on leak management techniques for water distribution system.” Water Resources Management, 32(10), 3247-3269.

Hamilton, S. and McKenzie, R. (2014). Water management and water loss. IWA Publishing.

Hoffman, M. D. and Gelman, A. (2014). “The no-U-turn sampler: Adaptively setting path lengths in Hamiltonian Monte Carlo.” Journal of Machine Learning Research.

Hu, Z., Chen, B., Chen, W., Tan, D., and Shen, D. (2021). "Review of model-based and data-driven approaches for leak detection and location in water distribution systems." Water Supply.

Hutton, C. J., Kapelan, Z., Vamvakeridou-Lyroudia, L., and Savić, D. A. (2014). "Dealing with Uncertainty in Water Distribution System Models: A Framework for Real-Time Modeling and Data Assimilation.” Journal of Water Resources Planning and Management, 140(2), 169-183.

Moser, G., Paal, S. G., and Smith, I. F. (2017). "Measurement system design for leak detection in hydraulic pressurized networks." Structure and Infrastructure Engineering, 13(7), 918-928.

OECD (2016). Water Governance in Cities. OECD Publishing, Paris, <https://www.oecdilibrary.org/content/publication/9789264251090-en>.

Peach, N., Basseville, M., and Nikiforov, I. V. (1995). "Detection of Abrupt Changes: Theory and Applications.." Journal of the Royal Statistical Society. Series A (Statistics in Society), 158(1).

Pérez, R., Puig, V., Pascual, J., Quevedo, J., Landeros, E., and Peralta, A. (2011). "Methodology for leakage isolation using pressure sensitivity analysis in water distribution networks." Control Engineering Practice, 19(10), 1157-1167.

Perez, R., Sanz, G., Puig, V., Quevedo, J., Cuguero Escofet, M. A., Nejjari, F., Meseguer, J., Cembrano, G., Mirats Tur, J. M., and Sarrate, R. (2014). "Leak localization in water networks: A model-based methodology using pressure sensors applied to a real network in barcelona [applications of control]." IEEE Control Systems.

Piller, O. (2019). “Water distribution system modeling and optimization.” French habilitation, Ecole doctorale Sciences des métiers de l'ingénieur, ED 432, Paris, France.

Piller, O., Elhay, S., Deuerlein, J., and Simpson, A. R. (2017). "Local sensitivity of pressuredriven modeling and demand-driven modeling steady-state solutions to variations in parameters." 
journal of Water Resources Planning and Management, 143(2).

Preis, A., Whittle, A. J., Ostfeld, A., and Perelman, L. (2011). "Efficient hydraulic state estimation technique using reduced models of urban water networks." journal of Water Resources Planning and Management, 137(4), 343-351.

Pudar, R. S. and Liggett, J. A. (1992). "Leaks in pipe networks.” journal of Hydraulic Engineering, $118(7), 1031-1046$.

Puust, R., Kapelan, Z., Savic, D. A., and Koppel, T. (2010). “A review of methods for leakage management in pipe networks." Urban Water journal, 7(1), 25-45.

Romano, M., Kapelan, Z., and Savić, D. A. (2013). "Geostatistical techniques for approximate location of pipe burst events in water distribution systems." Journal of Hydroinformatics, 15(3).

Rossman, L. A. (2000). Epanet 2 User 's Manual. US EPA.

Sanz, G., Pérez, R., Kapelan, Z., and Savic, D. (2016). "Leak detection and localization through demand components calibration." journal of Water Resources Planning and Management, 142(2), 04015057.

Savic, D. A., Kapelan, Z. S., and Jonkergouw, P. M. (2009). “Quo vadis water distribution model calibration?." Urban Water Journal.

Seabold, S. and Perktold, J. (2010). "Statsmodels: Econometric and statistical modeling with python." 9th Python in Science Conference.

Shumway, R. H. and Stoffer, D. S. (2010). Time Series Analysis and Its Applications With R Examples. Springer International Publishing, 4 edition.

Soldevila, A., Blesa, J., Tornil-Sin, S., Duviella, E., Fernandez-Canti, R. M., and Puig, V. (2016). "Leak localization in water distribution networks using a mixed model-based/data-driven approach.” Control Engineering Practice, 55, 162-173.

Soldevila, A., Fernandez-Canti, R. M., Blesa, J., Tornil-Sin, S., and Puig, V. (2017). "Leak localization in water distribution networks using bayesian classifiers." Journal of Process Control, $55,1-9$.

Steffelbauer, D. B., Blokker, E. J. M., Buchberger, S. G., Knobbe, A., and Abraham, E. (2021). 
"Dynamic time warping clustering to discover socioeconomic characteristics in smart water meter data." journal of Water Resources Planning and Management, 147(6), 04021026.

Steffelbauer, D. B., Deuerlein, J., Gilbert, D., Piller, O., and Abraham, E. (2020). “A Dual Model For Leak Detection and Localization." BattLeDIM: Battle of the Leakage Detection and Isolation Methods, Vrachimis, Eliades, Taormina, Ostfeld, Kapelan, Liu, Kyriakou, Pavlou, Qiu, and Polycarppou, eds., Beijing, China, Zenodo, <https://doi.org/10.5281/zenodo.3923907>.

Steffelbauer, D. B. and Fuchs-Hanusch, D. (2016a). "Efficient Sensor Placement for Leak Localization Considering Uncertainties.” Water Resources Management, 30(14), 5517-5533.

Steffelbauer, D. B. and Fuchs-Hanusch, D. (2016b). "Fitness landscapes and distance metrics for model-based leakage localization." 2016 3rd Conference on Control and Fault-Tolerant Systems (SysTol), IEEE, 343-348, <http://ieeexplore.ieee.org/document/7739774/>.

Storn, R. and Price, K. (1997). "Differential Evolution - A Simple and Efficient Heuristic for Global Optimization over Continuous Spaces." Journal of Global Optimization.

Vrachimis, S. G., Eliades, D. G., Taormina, R., Ostfeld, A., Kapelan, Z., Liu, S., Kyriakou, M. S., Pavlou, P., Qiu, M., and Polycarpou, M. (2020). "Dataset of BattLeDIM: Battle of the Leakage Detection and Isolation Methods." dataset, <https://doi.org/10.5281/zenodo.4017659> (September).

Wu, Z. Y. (2008). "Innovative Optimization Model for Water Distribution Leakage Detection." Bentley Methods Solution Center, 1-8. 


\section{List of Tables}

1 Original pipe characteristics in the INP file and corresponding cluster membership; in red the original external parameters that were corrected for PVC and PE pipes. . 30

2 Calibration of HW coefficients; the first three columns are the lower bound, initial estimate, and upper bound values for the six clusters; the last three columns are the $99 \%$ confidence intervals centered on the value at convergence; in bold the final point estimate. . . . . . . . . . . . . . . . . 31

3 Results of leak detection and localization: The true location, the start time and the maximum leakage outflow $\max \left(Q_{L}\right)$ are taken from the BattLeDIM solutions. The estimated location is found with the leak localization algorithm. $t_{D}$ is the detection time measured in hours since the true start time of the leak. The distance between the true and the estimated leak location is the shortest topological distance over the pipes in meter. Zone shows in which area of the network the leak is located. Perfectly located leaks are shown in boldface (plus minus $10 \mathrm{~m}$ ), while leaks with a distance greater than $300 \mathrm{~m}$ (missed leaks according to the BattLeDIM rules) are highlighted with an asterisk. . . . . . . . . . . . . . . 32 
TABLE 1. Original pipe characteristics in the INP file and corresponding cluster membership; in red the original external parameters that were corrected for PVC and PE pipes.

\begin{tabular}{l|c|c|c|c|r}
\hline $\begin{array}{l}\text { Diameter } \\
\text { in mm }\end{array}$ & C $_{H W}$ & Zone & $\begin{array}{c}\text { Cluster } \sharp \\
\text { in Eq. (3) }\end{array}$ & $\sharp$ pipes & $\begin{array}{r}\text { Length } \\
\text { in m }\end{array}$ \\
\hline $53.6(63)$ & 146 & $\mathrm{~A}$ & 1 & 3 & 71.40 \\
$53.6(63)$ & 146 & $\mathrm{~B}$ & 5 & 1 & 9.21 \\
$64(75)$ & 146 & $\mathrm{~A}$ & 1 & 1 & 60.08 \\
100 & 120 & $\mathrm{~A}$ & 2 & 76 & 3639.10 \\
100 & 120 & $\mathrm{~B}$ & 5 & 25 & 1190.11 \\
100 & 140 & $\mathrm{~A}$ & 3 & 500 & 24069.65 \\
100 & 140 & $\mathrm{C}$ & 6 & 104 & 5201.60 \\
150 & 120 & $\mathrm{~A}$ & 2 & 7 & 313.62 \\
150 & 140 & $\mathrm{~A}$ & 3 & 90 & 4102.87 \\
150 & 120 & $\mathrm{~B}$ & 5 & 6 & 226.56 \\
$141(160)$ & 146 & $\mathrm{~A}$ & 1 & 16 & 713.73 \\
200 & 90 & $\mathrm{~A}$ & 4 & 59 & 2749.71 \\
200 & 90 & $\mathrm{C}$ & 6 & 5 & 195.90 \\
$198.2(225)$ & 146 & $\mathrm{~A}$ & 1 & 12 & 558.58 \\
\hline
\end{tabular}


TABLE 2. Calibration of HW coefficients; the first three columns are the lower bound, initial estimate, and upper bound values for the six clusters; the last three columns are the $99 \%$ confidence intervals centered on the value at convergence; in bold the final point estimate.

\begin{tabular}{c|c|c|c|c|c|c}
\hline Cluster $\sharp$ & $\mathbf{x}^{L}$ & $\mathbf{x}^{0}$ & $\mathbf{x}^{U}$ & $\mathbf{x}^{11}-\Delta_{x}$ & $\mathbf{x}^{11}$ & $\mathbf{x}^{11}+\Delta_{x}$ \\
\hline 1 & 60 & $\mathbf{1 4 6}$ & 160 & 141.9 & 152.8 & 163.7 \\
2 & 60 & 120 & 160 & 108.1 & $\mathbf{1 0 9 . 7}$ & 111.3 \\
3 & 60 & 140 & 160 & 141.1 & $\mathbf{1 4 1 . 6}$ & 142.1 \\
4 & 60 & 90 & 160 & 126.5 & $\mathbf{1 2 6 . 8}$ & 127.1 \\
5 & 60 & 136 & 160 & 100.4 & $\mathbf{1 1 1 . 2}$ & 122.0 \\
6 & 60 & 133 & 160 & 133.1 & $\mathbf{1 3 4 . 0}$ & 134.9 \\
\hline
\end{tabular}


TABLE 3. Results of leak detection and localization: The true location, the start time and the maximum leakage outflow $\max \left(Q_{L}\right)$ are taken from the BattLeDIM solutions. The estimated location is found with the leak localization algorithm. $t_{D}$ is the detection time measured in hours since the true start time of the leak. The distance between the true and the estimated leak location is the shortest topological distance over the pipes in meter. Zone shows in which area of the network the leak is located. Perfectly located leaks are shown in boldface (plus minus $10 \mathrm{~m}$ ), while leaks with a distance greater than $300 \mathrm{~m}$ (missed leaks according to the BattLeDIM rules) are highlighted with an asterisk.

\begin{tabular}{|c|c|c|c|c|c|c|}
\hline $\begin{array}{l}\text { True Loc. } \\
\text { - }\end{array}$ & $\begin{array}{c}\text { start time } \\
-\end{array}$ & $\begin{array}{r}\max \left(Q_{L}\right) \\
\left(m^{3} / h\right)\end{array}$ & $\begin{array}{l}\text { Est. Loc. } \\
-\end{array}$ & $\begin{array}{r}t_{D} \\
(h)\end{array}$ & $\begin{array}{r}\text { Distance } \\
(m)\end{array}$ & $\begin{array}{c}\text { Zone } \\
-\end{array}$ \\
\hline p427 & 2018-02-13 08:25 & 5.11 & - & - & - & A \\
\hline p654 & 2018-07-05 03:40 & 5.49 & p654 & 956.33 & 0 & A \\
\hline p810 & 2018-07-28 03:05 & 6.91 & p810 & 668.92 & 0 & A \\
\hline p523 & 2019-01-15 23:00 & 28.39 & p500 & 0.00 & 205 & A \\
\hline p827 & 2019-01-24 18:30 & 26.46 & p827 & -0.08 & 0 & A \\
\hline p653 & 2019-03-03 13:10 & 18.28 & p655 & 273.42 & 106 & A \\
\hline p710 & 2019-03-24 14:15 & 5.58 & p702 & 0.00 & 222 & A \\
\hline p514 & 2019-04-02 20:40 & 15.58 & p226 & 0.00 & 90 & A \\
\hline $\mathrm{p} 331^{(*)}$ & 2019-04-20 10:10 & 10.93 & p905 & 0.00 & 355 & A \\
\hline p193 ${ }^{(*)}$ & 2019-05-19 10:40 & 10.36 & p185 & 417.33 & 398 & A \\
\hline p142 & 2019-06-12 19:55 & 27.04 & p623 & 0.00 & 2 & A \\
\hline p586 & 2019-07-26 14:40 & 20.52 & p586 & 215.50 & 0 & A \\
\hline p721 ${ }^{(*)}$ & 2019-08-02 03:00 & 13.18 & p703 & 222.92 & 354 & A \\
\hline p800 & 2019-08-16 14:00 & 21.95 & p820 & 110.50 & 196 & A \\
\hline p123 & 2019-09-13 20:05 & 9.19 & p201 & 588.33 & 133 & A \\
\hline p455 & 2019-10-03 14:00 & 11.05 & p109 & 584.92 & 142 & A \\
\hline p762 & 2019-10-09 10:15 & 15.71 & p745 & 301.00 & 179 & A \\
\hline $\mathrm{p} 426^{(*)}$ & 2019-10-25 13:25 & 13.56 & p42 & 0.00 & 779 & A \\
\hline p879 & 2019-11-20 11:55 & 10.93 & p884 & 342.50 & 256 & A \\
\hline p680 & 2019-07-10 08:45 & 5.37 & p680 & 0.00 & 0 & B \\
\hline p257 & 2018-01-08 13:30 & 6.87 & p257 & 104.50 & 0 & $\mathrm{C}$ \\
\hline p280 & 2019-02-10 13:05 & 5.26 & p251 & 0.00 & 49 & $\mathrm{C}$ \\
\hline $\mathrm{p} 277^{(*)}$ & 2019-05-30 21:55 & 7.36 & $\mathrm{p} 8$ & 541.83 & 358 & $\mathrm{C}$ \\
\hline
\end{tabular}




\section{List of Figures}

1 Overview of the hierarchical leak detection and isolation approach from left to right: Starting with the data analysis (measurements and EPANET model), then model calibration (nodal demand and pipe roughness), followed by simulations with the dual model approach, to finally detect and localize leaks. . . . . . . . . . . 35

2 Network colored by calibration clusters of Hazen-Williams roughness coefficients. Pressure measurements are shown as circles. In a) the roughness iterations are plotted ; in b), the inset shows the principle of the dual model, where the pressure measurements are replaced by the combination of a valve and a virtual reservoir whose head is equal to the measured head $h_{i}$; the dual model transforms $h_{i}$ into virtual leakage flows $q_{v_{i}}$; in (c) the pressure residuals are shown for the first week of 2018; and finally, in (d) the minimum, maximum, and root mean square errors (RMSE) are shown in increasing RMSE order. . . . . . . . . . . . . . . . 36

3 Weekly seasonality (a) and yearly trend (b) extracted from the AMR measurements for the different customer types (Residential and Commercial) and nodes consisting of a mix of them (Mixed). . . . . . . . . . . . . . 37

4 Leakage outflow in Area C (a) estimated by comparing the "virtual" inflow measurement and the demand model and (b) as provided by the dual model. . . . . . . 38

5 Dual model signals for first two leaks in Area A in 2019 (location at pipes p827 and p523 with magnitudes of approximately $27 \mathrm{~m}^{3} / \mathrm{h}$ each). (a) Pressure measurements $p$ over time, (b) sharp and localized signal of the virtual leak outflows $q_{v}$ over time calculated by the dual model at the same measurement locations, (c) the sum over all virtual leak flows in the dual model serves as good estimates for leak size. . . . 39

6 Snapshot of the leakage isolation tool: calculated outflows at virtual reservoirs of sensor nodes and correlation results: small diamonds for current time step and large diamonds for sum of all time steps (the size represents the score) . . . . . . . . . . 40 
7 Results of leak detection and localization for the unknown leaks in Area C in 2019:

(a) Identified leakage outflows over time and (b) estimated locations of the leaks. Crosses are the estimated leak locations, circles indicate the real locations. . . . . . 41

8 Results of leak detection and localization for the unknown leaks in Area B in 2019:

(a) Identified leakage outflows over time; and (b) estimated locations of the leaks. The Cross is the estimated leak location, the circle indicates the real location. . . . 42

9 Results of leak detection for the unknown leaks in Area A in 2019: (a) Leakage outflows for the first half of the year / leaks, and (b) for the second half of the year / leaks. The second half also includes the ongoing leaks from (a) as shaded lines. Additionally, the missed detected leak at pipe p427 is shown in (a) . . . . . . . . . 43

10 Results of leak localization for the unknown leaks in Area A in 2019: (a) First half of the year from January to June, and (b) for the second half of the year from July to December. The not detected leak at pipe 427 is shown as a white cross in (a). Crosses are the estimated leak locations, circles indicate the real locations. . . . . . 44 


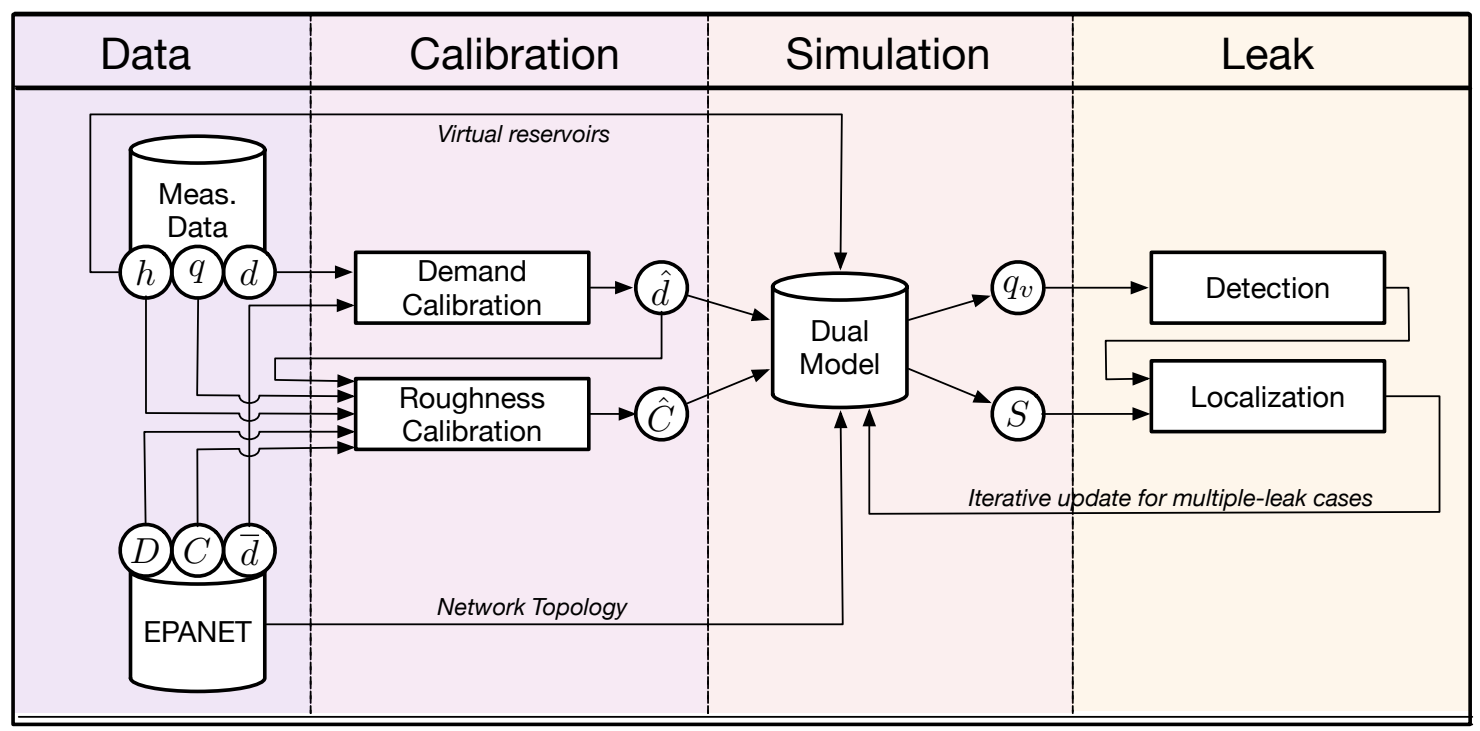

Fig. 1. Overview of the hierarchical leak detection and isolation approach from left to right: Starting with the data analysis (measurements and EPANET model), then model calibration (nodal demand and pipe roughness), followed by simulations with the dual model approach, to finally detect and localize leaks. 

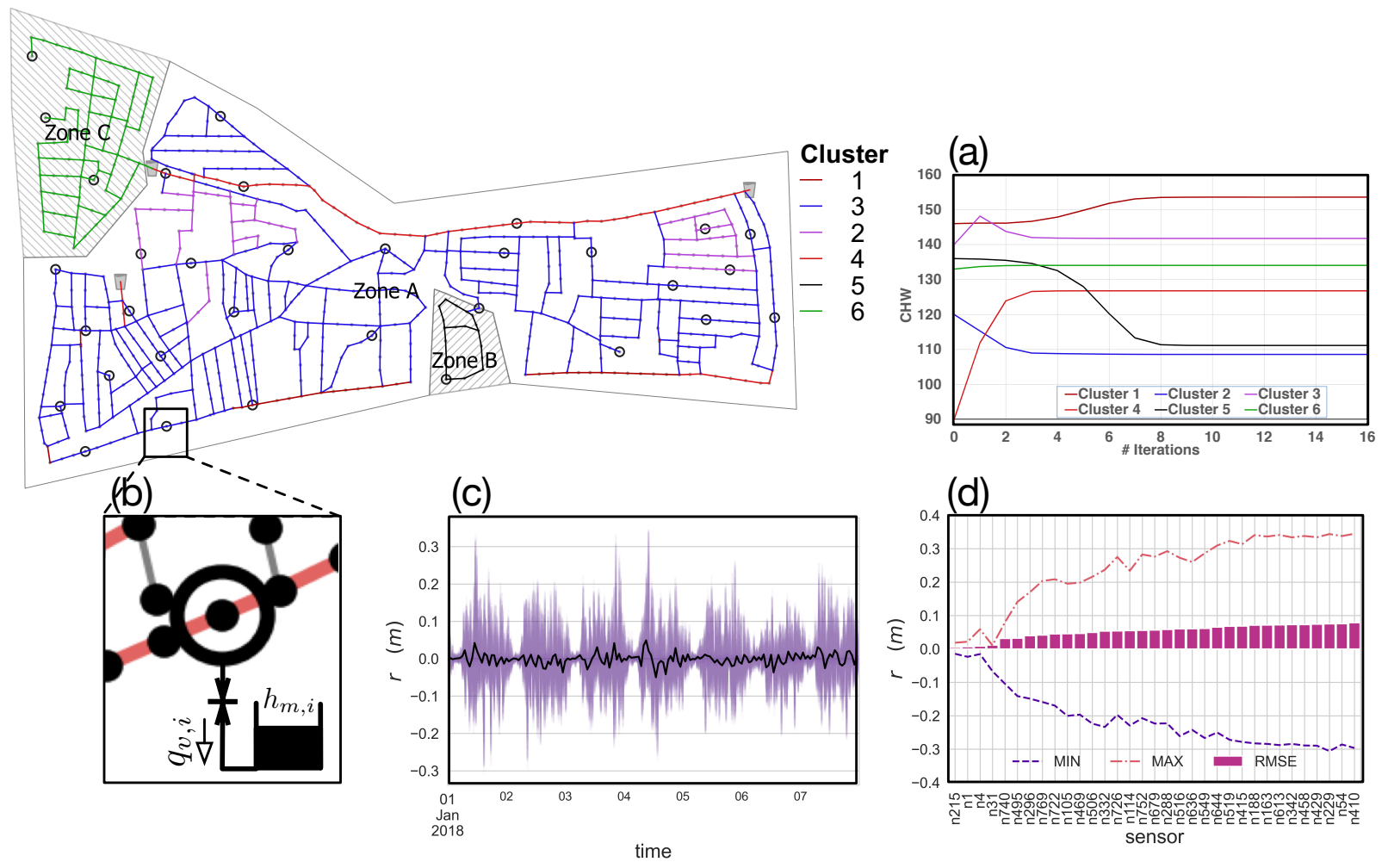

Fig. 2. Network colored by calibration clusters of Hazen-Williams roughness coefficients. Pressure measurements are shown as circles. In a) the roughness iterations are plotted; in b), the inset shows the principle of the dual model, where the pressure measurements are replaced by the combination of a valve and a virtual reservoir whose head is equal to the measured head $h_{i}$; the dual model transforms $h_{i}$ into virtual leakage flows $q_{v_{i}}$; in (c) the pressure residuals are shown for the first week of 2018; and finally, in (d) the minimum, maximum, and root mean square errors (RMSE) are shown in increasing RMSE order. 

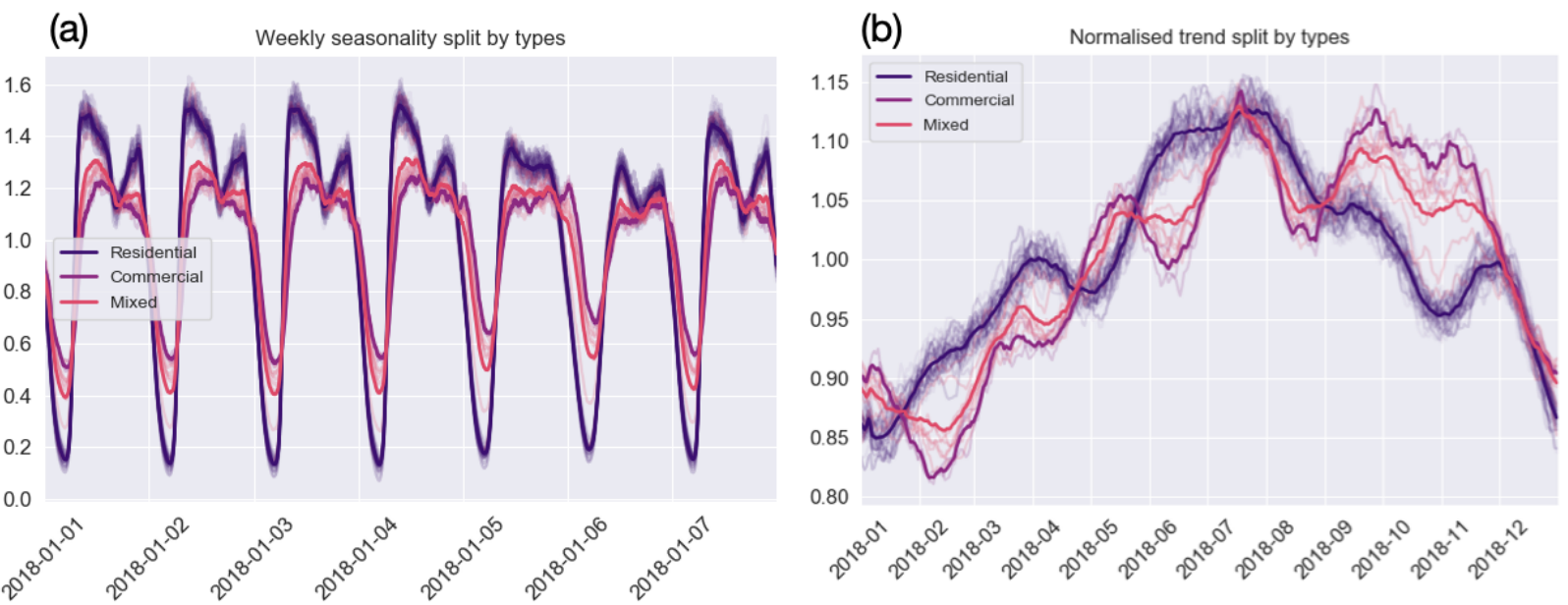

Fig. 3. Weekly seasonality (a) and yearly trend (b) extracted from the AMR measurements for the different customer types (Residential and Commercial) and nodes consisting of a mix of them (Mixed). 

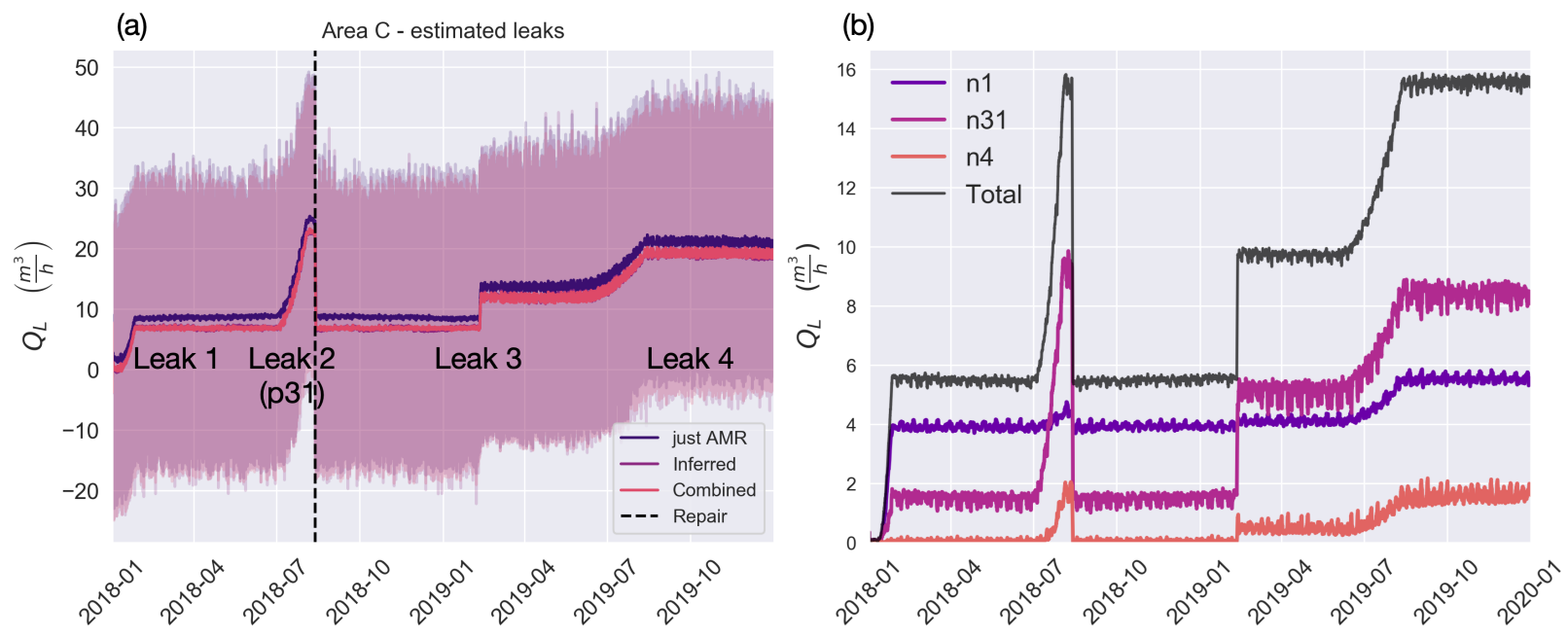

Fig. 4. Leakage outflow in Area C (a) estimated by comparing the "virtual" inflow measurement and the demand model and (b) as provided by the dual model. 

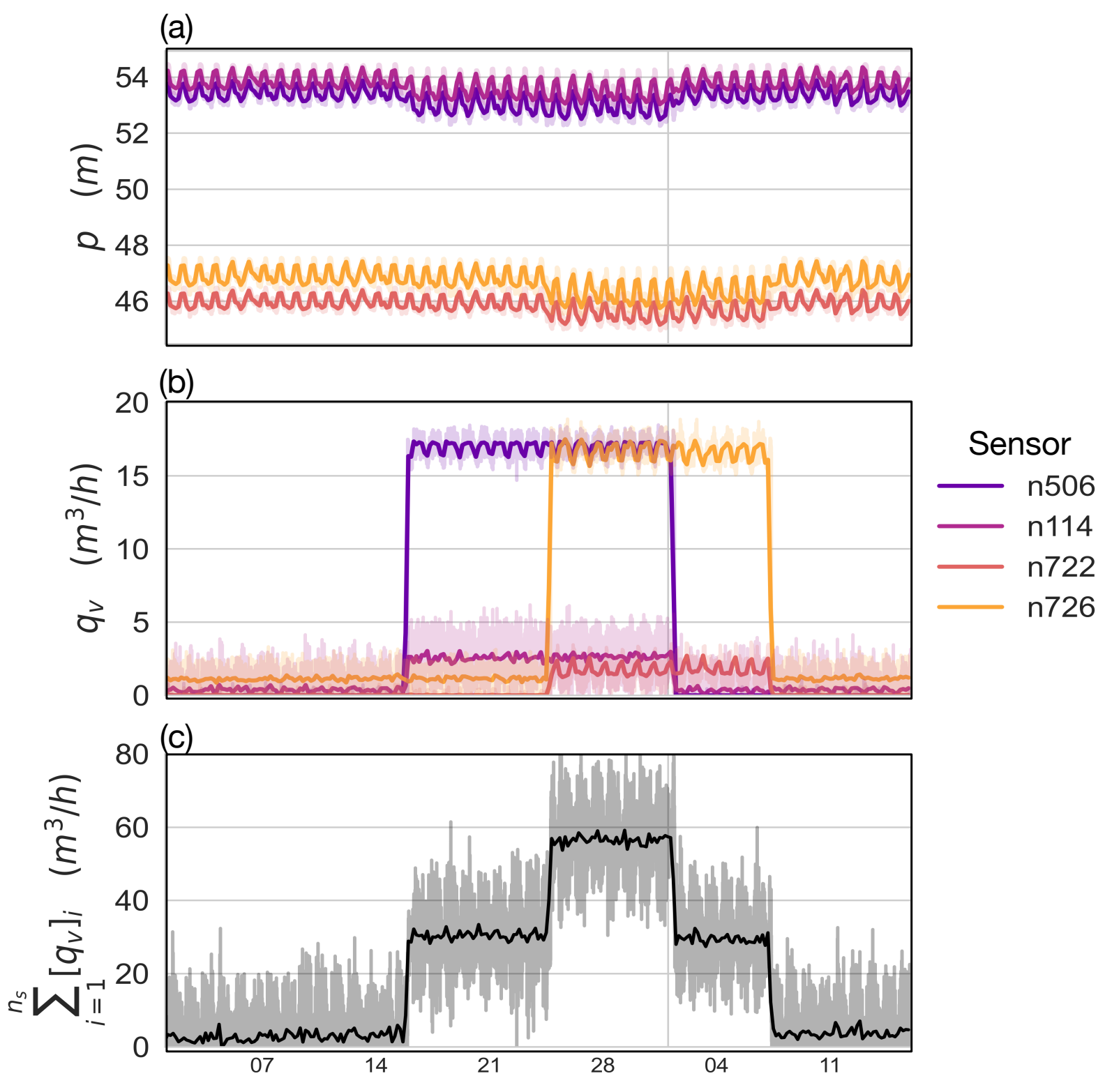

Jan

Feb 2019

\section{time}

Fig. 5. Dual model signals for first two leaks in Area A in 2019 (location at pipes p 827 and p523 with magnitudes of approximately $27 \mathrm{~m}^{3} / h$ each). (a) Pressure measurements $p$ over time, (b) sharp and localized signal of the virtual leak outflows $q_{v}$ over time calculated by the dual model at the same measurement locations, (c) the sum over all virtual leak flows in the dual model serves as good estimates for leak size. 


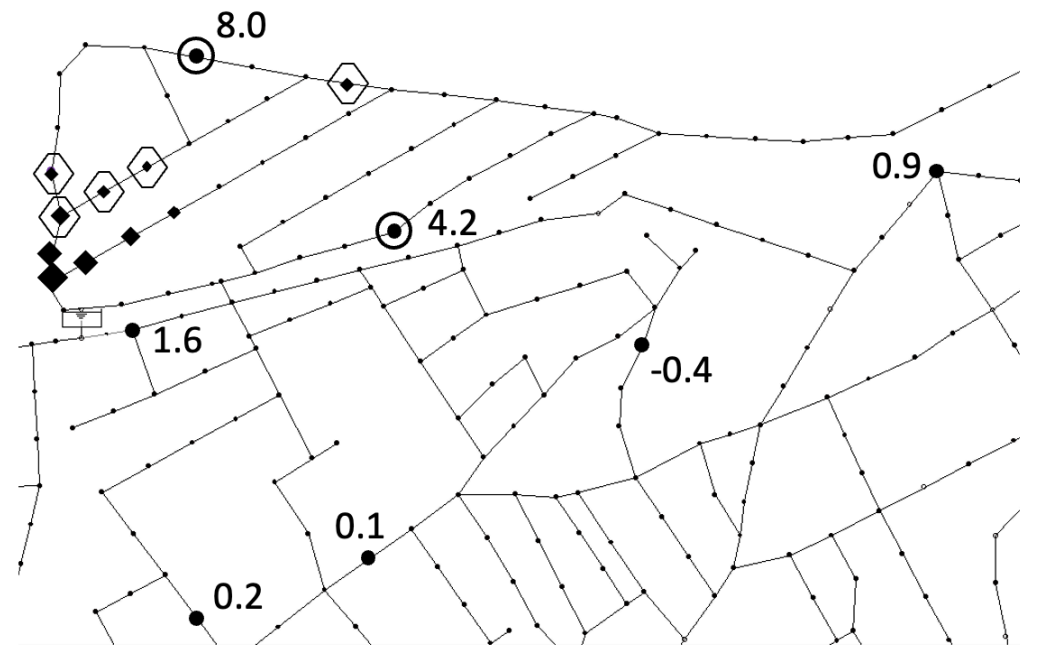
Nodes with highest
- correlations over all
time steps (size $=$ score)
Nodes with highest
- correlations at current time step
Sensor nodes with
0.1 calculated outflows [L/s]
Sensor nodes with
(-) calculated outflow above $5 \mathrm{~L} / \mathrm{s}$

Fig. 6. Snapshot of the leakage isolation tool: calculated outflows at virtual reservoirs of sensor nodes and correlation results: small diamonds for current time step and large diamonds for sum of all time steps (the size represents the score). 
(a)

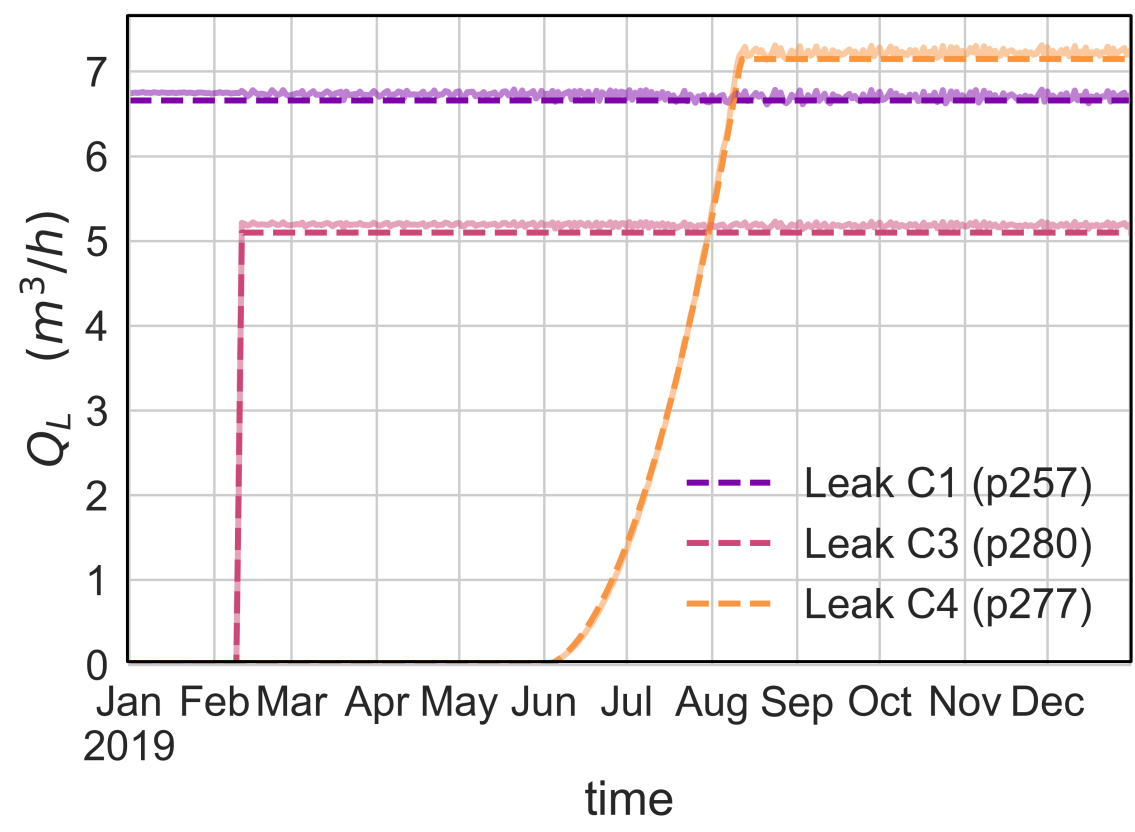

(b)

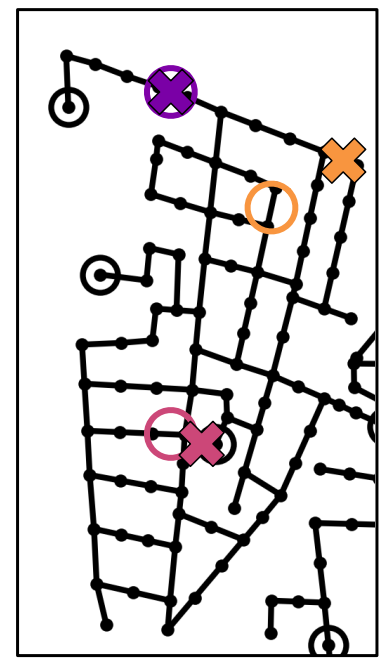

Fig. 7. Results of leak detection and localization for the unknown leaks in Area C in 2019: (a) Identified leakage outflows over time and (b) estimated locations of the leaks. Crosses are the estimated leak locations, circles indicate the real locations. 
(a)

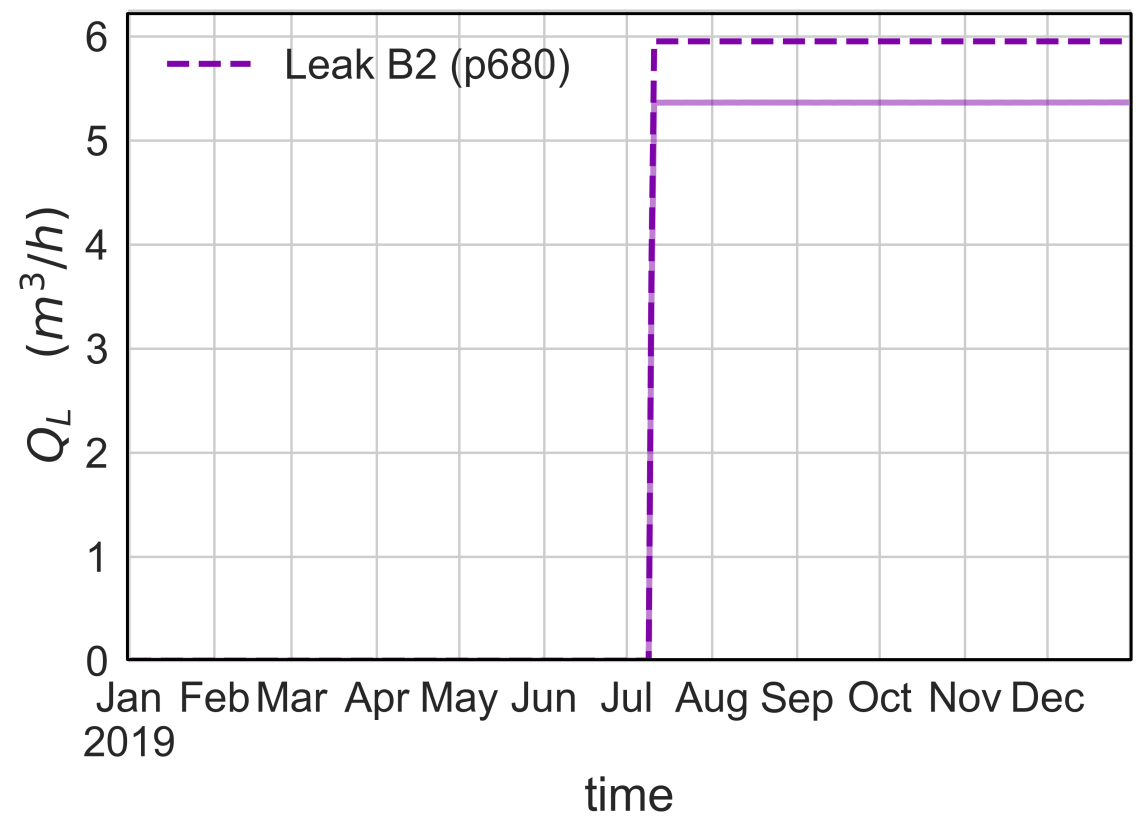

(b)

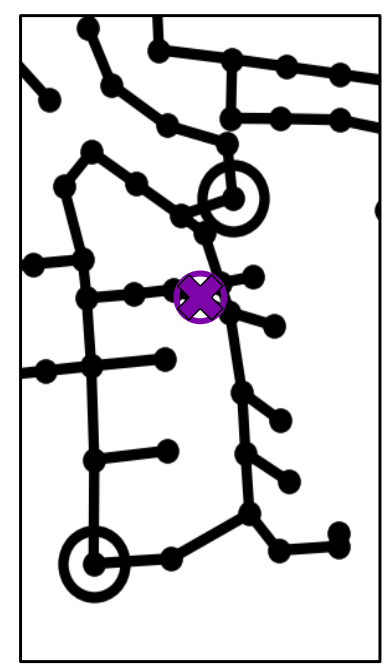

Fig. 8. Results of leak detection and localization for the unknown leaks in Area B in 2019: (a) Identified leakage outflows over time; and (b) estimated locations of the leaks. The Cross is the estimated leak location, the circle indicates the real location. 

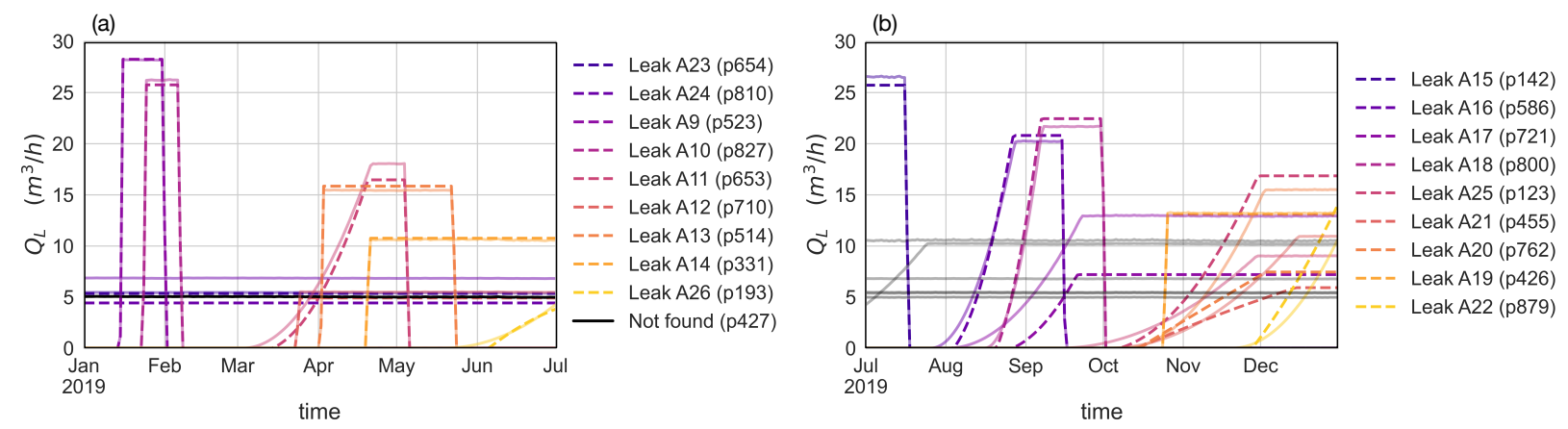

Fig. 9. Results of leak detection for the unknown leaks in Area A in 2019: (a) Leakage outflows for the first half of the year / leaks, and (b) for the second half of the year / leaks. The second half also includes the ongoing leaks from (a) as shaded lines. Additionally, the missed detected leak at pipe $\mathrm{p} 427$ is shown in (a). 
(a)

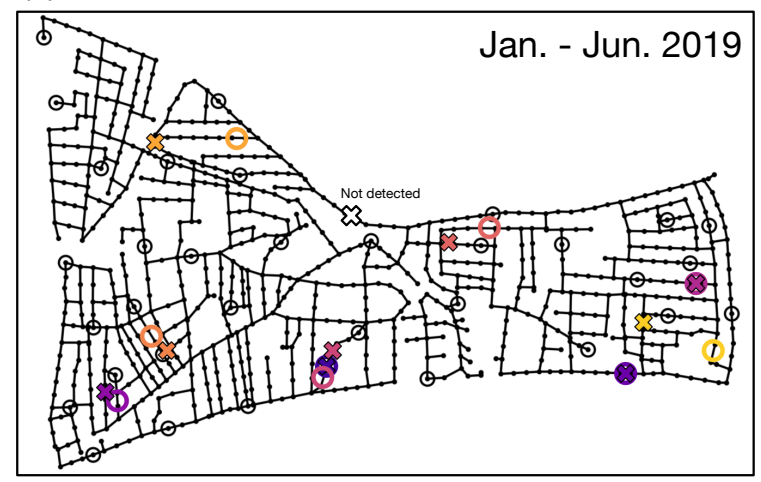

(b)

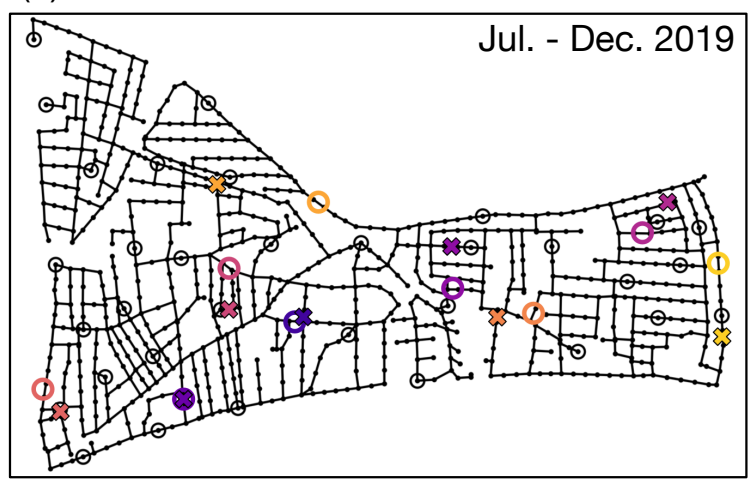

Fig. 10. Results of leak localization for the unknown leaks in Area A in 2019: (a) First half of the year from January to June, and (b) for the second half of the year from July to December. The not detected leak at pipe 427 is shown as a white cross in (a). Crosses are the estimated leak locations, circles indicate the real locations. 